Submitted to Journal of Statistical Physics: 12/28/95

\title{
MULTIFRACTAL DIMENSIONS FOR BRANCHED GROWTH
}

\author{
Thomas C. Halsey ${ }^{1}$, Katsuya Honda ${ }^{2}$, and Bertrand Duplantier ${ }^{3}$
}

\begin{abstract}
A recently proposed theory for diffusion-limited aggregation (DLA), which models this system as a random branched growth process, is reviewed. Like DLA, this process is stochastic, and ensemble averaging is needed in order to define multifractal dimensions. In an earlier work [T.C. Halsey and M. Leibig, Phys. Rev. A 46, 7793 (1992)], annealed average dimensions were computed for this model. In this paper, we compute the quenched average dimensions, which are expected to apply to typical members of the ensemble. We develop a perturbative expansion for the average of the logarithm of the multifractal partition function; the leading and sub-leading divergent terms in this expansion are then resummed to all orders. The result is that in the limit where the number of particles $n \rightarrow \infty$, the quenched and annealed dimensions are $i d e n t i c a l$; however, the attainment of this limit requires enormous values of $n$. At smaller, more realistic values of $n$, the apparent quenched dimensions differ from the annealed dimensions. We interpret these results to mean that while multifractality as an ensemble property of random branched growth (and hence of DLA) is quite robust, it subtly fails for typical members of the ensemble.
\end{abstract}

Keywords: Diffusion-limited aggregation, pattern formation, branched growth

1 Exxon Research and Engineering, Route 22 East, Annandale, N.J. 08801 U.S.A.

2 Department of Mathematics, Shinshu University, Matsumoto 390, Nagano Pref., Japan

3 Service de Physique Théorique, C.E. Saclay, 91191 Gif-sur-Yvette, France 


\section{Introduction}

Many natural growth processes generate branched structures. Probably the most celebrated such process is diffusion-limited aggregation (DLA). A simple algorithm for this type of growth process, introduced by Witten and Sander, has allowed over ten

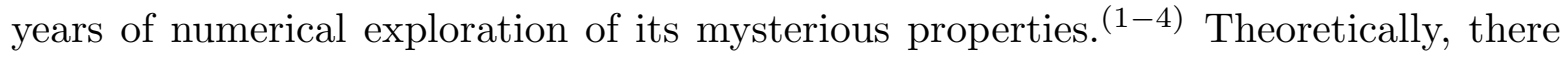
has been less progress. A number of studies have, with greater or lesser success, used real-space renormalization or variants thereof to study the self-similarity of DLA clusters. ${ }^{(5-7)}$ An alternative approach has been to try to deepen our understanding of the scaling structure of DLA clusters by emphasizing the "multifractal" nature of the growth of such clusters. ${ }^{(8-10)}$

Recently, a quite broad framework has been proposed to study branched growth processes. ${ }^{(11)}$ This framework relies upon the dynamics of competition between neighboring branches in these structures. For DLA, one of the authors of this work has proposed a method of determining the universal dynamics underlying this competition, a method which allows a priori computation of, e.g., the overall fractal dimension of DLA clusters. ${ }^{(12)}$ Results of this approach are in good agreement with numerical results for DLA.

In this work, we turn to the implications of this approach for the multifractal properties of DLA clusters. Our principal result is that fluctuations in the ensemble of possible DLA clusters seem to play a more important role than previously expected in determining these multifractal properties. In fact, while multifractality is well defined as an ensemble property of DLA, there appears to be a quite precise sense in which typical DLA clusters are not multifractals at all!

This result should be placed in the context of a number of studies claiming deviations from perfect fractal or multifractal behavior for DLA. A number of groups have reported anomalies in the scaling behavior of the very weak growth sites in DLA. (13) More recently, Mandelbrot and collaborators have proposed that the radius of gyration scaling of large DLA clusters may be more complicated than previously believed. ${ }^{(14)}$ However, in our case, the scaling anomalies correspond to the most active, strongly growing regions of the cluster, and the form of the anomalies can be computed. 


\section{Diffusion-limited aggregation}

The Witten-Sander algorithm is based on modelling growth as an aggregation of random walkers. Consider a cluster composed of $n$ particles. To generate the $n+1$ particle cluster, introduce a random walker at a great distance from the cluster. This random walker will either escape to infinity without encountering the cluster, or else will eventually encounter the cluster. In the Witten-Sander algorithm, the particle sticks at the point of its first contact with the cluster, thereby forming the $n+1$ particle cluster. The procedure is then iterated to create arbitrarily large clusters (the current record in two dimensions, with off-lattice random walks, is in the neighborhood of $10^{8}$ particles! $)^{(15)}$

Structures generated by this algorithm are highly branched and ramified (Figure 1). Fractal dimensions, defined by the scaling of the average cluster radius of gyration $r_{g}$ with particle number $n, n \propto r_{g}^{D_{f}}$, are $D_{f} \approx 1.71$ in spatial dimensionality $d=2$ and $D_{f} \approx 2.49$ in spatial dimensionality $d=3$. In higher dimensionalities, fractal dimensions are given approximately by the phenomenological formula $D_{f} \approx\left(d^{2}+\right.$ 1)/(d+1). ${ }^{(16)}$

DLA is observed to be a good model for a variety of natural growth processes, including electrodeposition, viscous fingering, and solidification. ${ }^{17}$ ) Variants of the model have been proposed to account for colloidal aggregation, dielectric breakdown, and the fracture of brittle media. ${ }^{(18)}$

Note that DLA is a stochastic process, due to the underlying stochastic nature of the random walks. Properly speaking, for fixed particle number $n$ there exists a large ensemble of clusters, each with a certain probability of appearing. If we (naively) suppose that each arriving particle can attach to any of the pre-existing particles, then there are $(n-1)$ ! different "genealogically distinct" (i.e., with respect to particle attachments) members of this ensemble.

This implies that quantities such as $D_{f}$ must be defined as ensemble averages, since not all possible members of the ensemble will have the same $D_{f}$. For instance, a possible, though unlikely, ensemble member consists of a long straight chain of particles, which has $D_{f}=1$. Unfortunately, researchers have rarely paid much attention to 
this aspect of the problem, and few numerical studies even carefully report the method of ensemble averaging used.

\section{Multifractality}

In a particular cluster of $n$ particles, there is a well-defined probability that the $n+1$ 'st particle will stick to any particular pre-existing particle. Indexing the particles by $i$, we write the probabilities of attachment $\left\{p_{i}\right\} ; p_{i}$ will equal zero for any particle inacessible to an approaching random walker. These quantities are very broadly distributed, being relatively large at the tips of clusters, where arriving particles are quite likely to attach, and extremely small deep inside the "fjords" of a cluster, where random walkers will almost never successfully arrive without having previously contacted the cluster. If the distribution of $\left\{p_{i}\right\}$ is multifractal, then we expect that

$$
\sum_{i=1}^{n} p_{i}^{q}=n^{-\sigma(q)}
$$

where the exponent function $\sigma(q)$, which is defined by this relation, should be asymptotically independent of particle number $n .^{(19),(20)}$ (Note that usually multifractality is defined in terms of the scaling of moments of $\left\{p_{i}\right\}$ with respect to the length scale $r_{g}$, and not particle number, defining an exponent function $\tau(q)$. We use $\sigma(q)$ because for our purposes particle number is a more convenient basis for defining the exponent function. We expect $\tau(q)=D_{f} \sigma(q)$.)

These exponent functions have well-known interpretations in terms of the distribution of $\left\{p_{i}\right\}$. We will temporarily follow the literature and use $\tau(q)$ rather than $\sigma(q)$. Suppose that for any particular $p_{i}$ a corresponding $\alpha_{i}$ is defined by $p_{i}=\left(a / r_{g}\right)^{\alpha_{i}}$, where $a$ is the particle diameter, and $r_{g}$ is the cluster radius of gyration. Then the total number of particles with $p_{i}$ in a range $d \alpha, N(\alpha) d \alpha$, defines a quantity $f(\alpha)$ via $N(\alpha)=\left(a / r_{g}\right)^{-f(\alpha)} ; f(\alpha)$ is independent of $r_{g}$ for multifractals. ${ }^{(20)}$ The quantity $f(\alpha)$ can be obtained by Legendre transformation of $\tau(q)$,

$$
\begin{aligned}
\alpha(q) & =\frac{d \tau(q)}{d q} ; \\
f(\alpha(q)) & =q \alpha(q)-\tau(q) .
\end{aligned}
$$


The values of $f$ and $\alpha$ at a particular value of $q$ satisfy the tangent condition

$$
\frac{d f(\alpha)}{d \alpha}=q
$$

By definition, $f(\alpha)$ is an intrinsically positive quantity, since $N(\alpha) \geq 1$.

Now we must modify these standard relations to account for the stochastic nature of DLA. One approach is to define the exponent function $\sigma(q)$ by a simple average of the moments; by analogy with statistical mechanics, we term this "annealed" averaging, and the resulting dimensions $\sigma_{\mathcal{A}}(q)$ "annealed" dimensions, ${ }^{(21)}$

$$
\left\langle\sum_{i=1}^{n} p_{i}^{q}\right\rangle=n^{-\sigma_{\mathcal{A}}(q)}
$$

where the brackets $\langle\cdots\rangle$ denote, here and elsewhere, averaging over the ensemble of DLA clusters. We can similarly define $\tau_{\mathcal{A}}(q)$ in terms of the scaling with respect to the cluster radius ${ }^{1} r_{g}$ by $\left\langle\sum_{i=1}^{n} p_{i}^{q}\right\rangle=\left(a / r_{g}\right)^{-\tau_{\mathcal{A}}(q)}$.

One advantage of this procedure is that the corresponding function $f(\alpha)$ retains a simple interpretation. Suppose that the expectation value of the number of particles with probabilites $p_{i}$ corresponding to the exponent $\alpha$ is $\langle N(\alpha)\rangle$. Direct application of Legendre transformation to Eq. (1.4) gives $\langle N(\alpha)\rangle \propto\left(a / r_{g}\right)^{-f(\alpha)}$, where $f(\alpha)$ obeys Eqs. (1.2-3), and can be thereby generated from $\tau_{\mathcal{A}}(q) \equiv D_{f} \sigma_{\mathcal{A}}(q)$. One difference with the non-stochastic case is that, since the expectation value of a number can take any non-negative value, $f(\alpha)$ can now be negative. Figure 2 shows characteristic $f(\alpha)$ functions for toy models, in the non-stochastic case and for stochastic models with annealed averaging. Typically in toy models, negative values of $f$ appear for $\alpha$ sufficiently far from the value of $\alpha, \alpha_{m}$, which corresponds to the maximum value of $f,\left(\alpha_{m}\right.$ represents the most frequently occuring value of the growth probability.) In terms of $\tau_{\mathcal{A}}(q)$ or $\sigma_{\mathcal{A}}(q)$, negative values of $f$ thus correspond to large absolute values of $q$.

1 Unfortunately, $r_{g}$ is also a stochastic quantity. The ambiguity this introduces is one argument for using $\sigma(q)$, defined in terms of $n$, for which there is no such ambiguity. 
The great drawback of annealed averaging arises if the quantity $\Omega(q)=\sum_{i} p_{i}^{q}$ is subject to large fluctuations between different ensemble members. Suppose that these fluctuations are log-normal, so that the probability $\mathcal{P}(\Omega) d \Omega$ that a particular ensemble member has $\sum_{i} p_{i}^{q}$ in the range $[\Omega, \Omega+d \Omega]$ is

$$
\mathcal{P}(\Omega) d \Omega=P_{0} \exp \left(\frac{(\log \Omega-\log \bar{\Omega})^{2}}{\bar{\Sigma}}\right) d \log \Omega .
$$

Clearly, the most likely value of $\log \Omega, \log \bar{\Omega}$, is not equal to $\log \langle\Omega\rangle .{ }^{(22)}$ This raises the possibility that observation of the scaling function $\sigma(q)$ for a typical DLA cluster may lead to a different result than $\sigma_{\mathcal{A}}(q)$. However, Eq. (1.5) shows that if the fluctuations of $\Omega$ are $\log$-normal, then $\langle\log \Omega\rangle=\log \bar{\Omega}$. This suggests that dimensions $\sigma_{\mathcal{Q}}(q)$ defined through "quenched" averaging, ${ }^{(21)}$

$$
\left\langle\log \sum_{i=1}^{n} p_{i}^{q}\right\rangle \equiv-\sigma_{\mathcal{Q}}(q) \log n,
$$

may be closer to the typical result of an observation on, for instance, a numerically generated cluster. Of course, it is not necessarily the case that the fluctuations of $\Omega$ for DLA are log-normal. However, whatever the nature of these fluctuations, quenched averaging reduces the impact of rare ensemble members, and thus should yield results closer to the behavior of typical ensemble members.

If $\sigma_{\mathcal{Q}}(q)$ gives results characteristic of a typical member of the ensemble, then the corresponding $f(\alpha)$ cannot include negative values of $f$, which can only be interpreted as ensemble average quantities. ${ }^{(23)}$ Thus if the fluctuations of the ensemble are strong enough to lead to negative values of $f$ in $\sigma_{\mathcal{A}}(q)$, then we also expect that $\sigma_{\mathcal{A}}(q) \neq$ $\sigma_{\mathcal{Q}}(q)$.

This does not imply that $f<0$ are necessarily unobservable. Suppose at some value of $q, f(\alpha(q))$ (annealed) is $<0$. Then in order to see this value of $\alpha$, one must average over an ensemble of at least $N \sim\left(r_{g} / a\right)^{|f|}$ clusters. This will become difficult as sizes increase, but for small absolute values of $f$, negative values of $f$ should be observable. 
It is convenient to introduce the "partition sum" $\mathcal{Z}(q, \sigma, n)$ which is defined for a specific cluster by

$$
\mathcal{Z}(q, \sigma, n)=n^{\sigma} \sum_{i=1}^{n} p_{i}^{q}
$$

The definitions above are equivalent to requiring that the annealed dimensions satisfy

$$
\left\langle\mathcal{Z}\left(q, \sigma_{\mathcal{A}}(q) ; n\right)\right\rangle=1,
$$

and that the quenched dimensions satisfy

$$
\left\langle\log \mathcal{Z}\left(q, \sigma_{\mathcal{Q}}(q) ; n\right)\right\rangle=0,
$$

in the limit of large $n$. By definition, $\langle\mathcal{Z}(q, \sigma ; n)\rangle=n^{\sigma} Z(q, n)$, so that

$$
\langle\log \mathcal{Z}(q, \sigma ; n)\rangle=\sigma \log n+F(q, n),
$$

where $F$ is independent of $\sigma$.

\section{Branched growth model}

The branched growth model introduced in Ref. 11 is based upon an analysis of the dynamics of competition between neighboring branches. In standard off-lattice DLA algorithms, any particle that aggregates to a cluster has a unique "parent" particle, to which it attaches. Since no particle has two parents, no loops can develop in the structure. The position of any particular particle can be uniquely identified by specifying which branch it lies upon, on a succession of increasing length scales.

Consider a branch point, which is any particle that is parent to more than one succeeding particle. At a particular point in the development of the cluster, the two (higher numbers are possible, but are both unlikely and irrelevant) sub-branches coming off of this particle have respectively total masses $n_{1}, n_{2}$ and total growth probabilities $p_{1}, p_{2}$. These growth probabilities represent the total probability that the next 
particle will stick to any constituent of that particular sub-branch. We define relative masses and relative growth probabilities by

$$
\begin{aligned}
& x=\frac{p_{1}}{p_{1}+p_{2}} \equiv \frac{p_{1}}{p_{b}} \\
& y=\frac{n_{1}}{n_{1}+n_{2}} \equiv \frac{n_{1}}{n_{b}}
\end{aligned}
$$

where we have also defined total branch mass and growth probability $n_{b}, p_{b}$, each made up of a contribution from the two sub-branches.

Simple kinematics shows that, neglecting fluctuations in the numbers of particles arriving at this branch, we have $\mathrm{e}^{(11)}$

$$
\frac{d y}{d \log n_{b}}=x-y
$$

If $d x / d \log n_{b}$ can be expressed as a function of $x$ and $y$ alone, then there is a closed dynamics for branch competition as a function of these two variables. In Ref. 12, it was shown that by averaging over the intrinsic stochasticity of the DLA problem, a function $g(x, y)$ can be determined (at least in $d=2$ ) such that

$$
\frac{d x}{d \log n_{b}}=g(x, y),
$$

with $g(x, y)$ a calculable function of $x$ and $y$ alone. By symmetry, $g(x, y)=-g(1-$ $x, 1-y)$, so that there is a fixed point of the dynamics at $(x, y)=(1 / 2,1 / 2)$. For the computed function $g(x, y)$, this is a hyperbolically unstable fixed point, with one stable and one unstable direction. (Actually, this qualitative feature can also be predicted on general grounds, without computation). The unstable manifold of the fixed point leads to two other (stable) fixed points, at $(x, y)=(0,0)$ and $(x, y)=(1,1)$. Thus, sub-branch pairs, which are born in a tip-splitting process, compete in an unstable fashion, so that asymptotically one of the two sub-branches possesses virtually all of the mass and all of the growth probability of the pair.

Although the dynamics of a branch pair in the $x-y$ plane is thus deterministic, the stochasticity of the DLA problem is retained since the original position of a branch pair in this plane, i.e., when $n_{b} \sim 1$, and a tip-splitting event creates the pair, is a 
random function. In fact, sub-branch pairs born in most sections of the $x-y$ plane will be quickly driven to one or the other of the stable fixed points, at which one of the two sub-branches completely dominates the other. In Eqs. (1.12-13), we see that for general values of $x, y$, the fundamental scale over which $x$ and $y$ will change their values is $\log n_{b} \sim 1$, i.e., while the branch as a whole is still of microscopic size.

However, if the sub-branch pair is originally created quite close to the central, unstable fixed point, then it will remain in the vicinity of that fixed point up to larger values of $\log n_{b}$. Such pairs correspond to the large, relatively equal branch pairs seen in a DLA cluster. Thus, the large scale cluster structure is sensitive only to the distribution of birth probability in the immediate vicinity of the unstable fixed point. This probability distribution will be determined by the microscopic dynamics of tipsplitting, which do not recognize any special role of the unstable fixed point. Thus we expect this probability distribution to be only slowly varying near that point; it can be approximated for our purposes by a constant.

This criterion, and the specific form of $g(x, y)$, defines the random branched growth model. Computations with this model are much simplified by the fact that the branch competition trajectories born quite close to the stable manifold, which correspond to the large-scale structure, will be quickly drawn onto the unstable manifold. This manifold can thus be parameterized by $x$ and $y$ as functions of $\log n_{b}$, or of $n_{b}$. Of course, the manifold is symmetric about the unstable fixed point. It is convenient to introduce a parameter $\epsilon$, which parameterizes the original distance (when $n_{b} \sim 1$ ) of the branch pair from the stable manifold in the $x-y$ plane. If this distance is $\epsilon^{\nu}$, where $\nu$ is the eigenvalue along the unstable manifold at the unstable fixed point of the dynamics defined by Eqs. (1.12-13), it can be shown that $x$ and $y$ are functions of the combination $\epsilon n_{b}$, and that $\epsilon$ plays no further role in the dynamics. ${ }^{(11)}$

For practical purposes, we must thus specify $x\left(\epsilon n_{b}\right), y\left(\epsilon n_{b}\right)$ in order to compute with our model. A useful toy model, "Model Z" is displayed in Figure 3. In this model, the unstable manifold follows a straight line away from the fixed point until it strikes the line $x=0$ (or $x=1$ ). It then moves vertically into the stable fixed points. The angle of the unstable manifold at the fixed point is the one adjustable parameter for this type of trajectory. 
For more realistic purposes, we can use the unstable manifold computed for DLA in two dimensions in Ref. 12. ${ }^{(24)}$ This manifold is displayed in Figure 4.

\section{Summary of results}

In Ref. 11, it was shown that the annealed dimensions $\sigma_{\mathcal{A}}(q)$ for a branched growth model are determined by the simple implicit formula

$$
\int_{0}^{\infty} d \eta \eta^{\nu-1}\left\{\frac{x^{q}(\eta)}{y^{\sigma}(\eta)}+\frac{[1-x(\eta)]^{q}}{[1-y(\eta)]^{\sigma}}-1\right\} \equiv \int_{0}^{\infty} d \eta \eta^{\nu-1} \psi(\eta ; q, \sigma)=0
$$

where $\eta \equiv \epsilon n_{b}$ parameterizes position on the unstable manifold described in the above discussion. We have also defined the quantity $\psi(\eta ; q, \sigma)$ by

$$
\psi(\eta ; q, \sigma) \equiv \frac{x^{q}(\eta)}{y^{\sigma}(\eta)}+\frac{[1-x(\eta)]^{q}}{[1-y(\eta)]^{\sigma}}-1
$$

This quantity will appear frequently in our results. For model Z or for the computed DLA trajectories, Eq. (1.14) leads for $q>0$ to an annealed multifractal spectrum with the expected properties, such as negative values of $f(\alpha)$ for large values of $q$. For $q<0$, annealed multifractal dimensions are typically not well-defined, due to a divergence of the left-hand side in Eq. (1.14).

In this work, we study the structure of $\langle\log \mathcal{Z}\rangle$ for the random branched growth model, in order to determine the quenched multifractal dimensions for these models. We develop a diagrammatic perturbation expansion for this quantity, and we show how sets of terms in this expansion can be resummed to all orders. The form of the resulting expression is

$$
\begin{aligned}
\langle\log \mathcal{Z}(q, \sigma, n)\rangle=\left(\int d \eta \eta^{\nu-1} \psi(\eta ; q, \sigma)\right) & \Gamma_{0}(q, \sigma) \log n \\
& +\Gamma_{1}(q, \sigma)\left(1-n^{-\Delta(q)}\right)+\cdots
\end{aligned}
$$

where the exponent $\Delta(q)$ governing the finite-size corrections is positive and calculable. Because $\langle\log \mathcal{Z}\rangle=\sigma \log n+F(q, n)$, the power law terms should be independent of 
$\sigma$; we retain an ersatz dependence on $\sigma$ in Eq. (1.14) because this independence is difficult to see directly within our perturbative method. In the limit $n \rightarrow \infty$, the power-law and constant terms can be disregarded; thus it appears that the criterion $\int d \eta \eta^{\nu-1} \psi(\eta ; q, \sigma)=0$ is sufficient to determine $\sigma_{\mathcal{Q}}(q)$. Since this is the same criterion as that for the annealed dimensions $\sigma_{\mathcal{A}}(q)$, this implies that $\sigma_{\mathcal{Q}}(q)=\sigma_{\mathcal{A}}(q)$. This result is somewhat surprising, and also undermines the interpretation of $\sigma_{\mathcal{Q}}(q)$ as a property of a typical cluster, since we expect $\sigma_{\mathcal{A}}(q)$ to include regions of negative $f(\alpha)$.

This paradox is resolved by a further feature of this result. It concerns the order of the limits $q \rightarrow \infty, n \rightarrow \infty$. Negative values of $f$ appear for large values of $q$; however, $\lim _{q \rightarrow \infty} \Delta(q)=0$. Thus, for large $q$, the power-law terms in Eq. (1.16) do contribute logarithms to the result, provided that $n$ is not too large. Thus for moderate values of $n$, a different result is obtained for $\sigma_{\mathcal{Q}}(q)$, which proves not to include negative values of $f$. At large $n$, one will eventually cross over to the asympotic regime in which $\sigma_{\mathcal{Q}}(q)=\sigma_{\mathcal{A}}(q)$, but this value of $n$, which we term $n_{c}$, grows very quickly with $q$, as $n_{c}(q) \sim \exp \left(a e^{b q}\right)$, with $a$ and $b$ positive constants, as shown below explicitly in section 4 for a specific model, model Z. Thus for practically accessible values of $n$, we expect never to see negative values of $f$.

Of course, this resolution also implies that any typical cluster of finite size $n$ should probably not be viewed as a true multifractal, as its exponent function $\sigma_{\mathcal{Q}}(q)$ actually represents different scaling behaviors above and below the value of $q$ for which $n=n_{c}(q)$. One conclusion of our study is thus that multifractality should be properly viewed as an ensemble property of DLA, and not a property of individual DLA clusters.

This paper is comprised of five sections and five appendices. In section 2, we develop the perturbative expansion of $\langle\log \mathcal{Z}\rangle$. In section 3, we show how families of terms within this expansion can be resummed to all orders. In section 4 , we discuss the implications of these results for the multifractality of DLA clusters, and we compare our analytical results with numerical results for random branched growth. In section 5, we conclude and summarize. In appendix A, we show how to perform sums that play the role of "propagators" in our perturbative expansion. In appendix B, we list perturbation theory terms, with their diagrammatic expressions, up to fourth order. In appendix $\mathrm{C}$, we list some mathematical identities that are useful in the resummation 
of section 3. In appendix D we show a simpler method of obtaining some of the terms in the final expression for $\langle\log \mathcal{Z}\rangle$. Finally, in appendix E, we show how the expansion of section 2 can be recast as a perturbation theory in $q-1$.

\section{Perturbative expansion for $\langle\log \mathcal{Z}\rangle$}

Formally, we can write

$$
\langle\log \mathcal{Z}(q, \sigma, n)\rangle=\sum_{N=1}^{\infty} \frac{(-1)^{N-1}}{N}\left\langle(\mathcal{Z}-1)^{N}\right\rangle
$$

so that our problem is one of computing expectation values of powers of $\mathcal{Z}-1$. We know that

$$
\mathcal{Z}(q, \sigma, n)=n^{\sigma} \sum_{i=1}^{n} p_{i}^{q}=\sum_{i=1}^{n} \frac{p_{i}^{q}}{(1 / n)^{\sigma}}
$$

where we recall that the index $i$ labels the particles.

In our model, the only sites which are allowed to grow are at the end-points of the branching process. Thus we use Eq. (2.2), substituting a sum over these "elementary" sub-branches for the sum over particles $i$ in (2.2). Consider a particular elementary sub-branch of the cluster, of total growth probability $p_{e}$ and total mass $n_{e}$ (Figure 5). This sub-branch branches off from its sibling at a particular node, indexed by $\mathrm{J}$; the total number of particles in the elementary sub-branch and in its sibling taken together is $n_{J}$. If the elementary sub-branch is the weaker (stronger) of the two sub-branches, then it has a proportion $x\left(\epsilon_{J} n_{J}\right)$ (or $\left.1-x\left(\epsilon_{J} n_{J}\right)\right)$ of the total growth probability of the two sub-branches taken together, and a proportion $y\left(\epsilon_{J} n_{J}\right)$ (or $\left.1-y\left(\epsilon_{J} n_{J}\right)\right)$ of the total mass of the two sub-branches taken together. Here $\epsilon_{J}$ is the random variable specifying the original state for the branch point indexed by $J$ when the total number of descendants of that branch point was $n_{J}=1$. We will sometimes refer to the path that leads through the stronger of the two sub-branches as the "main branch", the weaker sub-branch corresponds to a "side-branching."

Since $\mathcal{Z}$ can be expressed in such a way that $x$ and $y$ always appear together, it is convenient to define quantities $f_{ \pm}(\epsilon n)$ by 


$$
\begin{aligned}
f_{-}(\eta) & \equiv \frac{x^{q}(\eta)}{y^{\sigma}(\eta)} \\
f_{+}(\eta) & \equiv \frac{(1-x(\eta))^{q}}{(1-y(\eta))^{\sigma}} .
\end{aligned}
$$

The contribution of our particular sub-branch to $\mathcal{Z}$ can be written as

$$
\frac{p_{e}^{q}}{\left(n_{e} / n\right)^{\sigma}}=\prod_{j=1}^{J} f_{\mu_{j}}\left(\epsilon_{j} n_{j}\right)
$$

where $\mu_{j}= \pm$, depending on whether the stronger or weaker branch is taken at the $j$ 'th node. The index $j$ indexes the nodes between the root of the cluster, $j=1$, and the elementary sub-branch, for which we have taken $j=J$. Note that $n_{1} \equiv n$, the total number of particles in the cluster. Note also that the index $j$ measures position upon a particular path from the root to an elementary sub-branch, so its meaning depends upon the exact sequence of $\left\{\mu_{j}\right\}$ chosen. Comparing with Eq. (2.2), we see that in Eq. (2.4) we are introducing a slightly different cut-off procedure than that used in Eq. (2.2), where $n_{e}=1$.

Averaging the quantity appearing on the right-hand side in Eq. (2.4) is difficult because $n_{j}$ is actually a function of all $\epsilon_{k}$ with $k<j$, since either $n_{j}=y\left(\epsilon_{j-1} n_{j-1}\right) n_{j-1}$, if at the $j$ 'th node we take the weaker of the two sub-branches originating at that node, or else $n_{j}=\left(1-y\left(\epsilon_{j-1} n_{j-1}\right)\right) n_{j-1}$, if we take the stronger of the two sub-branches. Thus the individual terms in the product appearing in Eq. (2.4) cannot be averaged independently of one another.

Nevertheless, it is instructive to investigate how a single one of these terms averages. Consider $f_{-}\left(\epsilon_{j} n_{j}\right)$. The average of this over the random variable $\epsilon_{j}$ is given by

$$
\left\langle f_{-}\left(\epsilon_{j} n_{j}\right)\right\rangle_{\epsilon_{j}}=\int_{0}^{\infty} d \epsilon_{j} \rho\left(\epsilon_{j}\right) f_{-}\left(\epsilon_{j} n_{j}\right)
$$

where \langle\rangle$_{\epsilon_{j}}$ denotes averaging over $\epsilon_{j}$, and $\rho\left(\epsilon_{j}\right)$ is the probability distribution for $\epsilon_{j}$. In the introduction, we stated that the initial distance in the $x-y$ plane from the unstable manifold is proportional to $\epsilon^{\nu}$. In addition, we stated that the probability 
distribution with respect to the distance measure $d \epsilon^{\nu}$ should be constant close to the unstable manifold. Since $d \epsilon^{\nu} \propto \epsilon^{\nu-1} d \epsilon$, we see that

$$
\lim _{\epsilon \rightarrow 0} \rho(\epsilon)=\rho_{0} \epsilon^{\nu-1}
$$

where $\rho_{0}$ is a constant. (The reader may consult Ref. 11 for a more detailed discussion of this point.) Now suppose that $n_{j} \gg 1$. The quantities $x(\eta), y(\eta) \rightarrow 0$ for $\eta \gg 1$, (see Figures 3 and 4 ), and for physical values of $\sigma$, we thus expect $f_{-}(\eta) \rightarrow 0$ for $\eta \gg 1$. Thus we have

$$
\int_{0}^{\infty} d \epsilon_{j} \rho\left(\epsilon_{j}\right) f_{-}\left(\epsilon_{j} n_{j}\right) \approx \frac{\rho_{0}}{n_{j}^{\nu}} \int_{0}^{\infty} d \eta \eta^{\nu-1} f_{-}(\eta) \equiv \frac{\rho_{0}}{n_{j}^{\nu}}\left\langle\left\langle f_{-}\right\rangle\right\rangle,
$$

where we have defined a normalized expectation value $\langle\langle\cdots\rangle\rangle$ by

$$
\langle\langle f(\eta)\rangle\rangle \equiv \int_{0}^{\infty} d \eta \eta^{\nu-1} f(\eta) .
$$

This expectation value does not depend on $n_{j}$, nor on anything else except the form of the unstable manifold. The corrections to Eq. (2.7) are of higher order in $n_{j}^{-1}$. Ignoring these corrections, we write

$$
\left\langle f_{-}\left(\epsilon_{j} n_{j}\right)\right\rangle=\left\langle\frac{\rho_{0}}{n_{j}^{\nu}}\right\rangle\left\langle\left\langle f_{-}\right\rangle\right\rangle
$$

where the remaining true expectation value $\left\langle n_{j}^{-\nu}\right\rangle$ depends only upon random variables $\epsilon_{k}$ with $k<j$.

The averaging of $f_{+}$is performed rather differently, because $\lim _{\eta \rightarrow \infty} f_{+}(\eta)=1$. Thus

$$
\begin{aligned}
\int_{0}^{\infty} d \epsilon_{j} \rho\left(\epsilon_{j}\right) f_{+}\left(\epsilon_{j} n_{j}\right) & =\int_{0}^{\infty} d \epsilon_{j} \rho\left(\epsilon_{j}\right)\left[1+\left(f_{+}\left(\epsilon_{j} n_{j}\right)-1\right)\right] \\
& =1+\int_{0}^{\infty} d \epsilon_{j} \rho\left(\epsilon_{j}\right)\left(f_{+}\left(\epsilon_{j} n_{j}\right)-1\right) \\
& \approx 1+\frac{\rho_{0}}{n_{j}^{\nu}}\left\langle\left\langle\left(f_{+}-1\right)\right\rangle\right\rangle \\
& \equiv 1+\frac{\rho_{0}}{n_{j}^{\nu}}\left\langle\left\langle g_{+}\right\rangle\right\rangle
\end{aligned}
$$


where we have used $\int_{0}^{\infty} d \epsilon \rho(\epsilon)=1$, and have introduced

$$
g_{+}(\eta) \equiv f_{+}(\eta)-1
$$

It is now natural to expand $\langle\log \mathcal{Z}\rangle$ perturbatively in $f_{-}$and $g_{+}$. Formally, we can associate a dummy parameter $\delta$ to each factor of $f_{-}$or $g_{+}$, expand order by order in $\delta$, and set $\delta=1$ at the end of the computation.

\section{Zeroth order in $\delta$}

At zeroth order in $\delta$, only one elementary sub-branch in the cluster contributes, that in the stronger sub-branch at each node, starting at the root (Figure 6). The contribution $p_{e}^{q} /\left(n_{e} / n\right)^{\sigma}$ from this sub-branch is given by

$$
\frac{p_{e}^{q}}{\left(n_{e} / n\right)^{\sigma}}=\prod_{j=1}^{J} f_{+}\left(\epsilon_{j} n_{j}\right)=\prod_{j=1}^{J}\left(1+g_{+}\left(\epsilon_{j} n_{j}\right)\right)
$$

where the index $j$ now denotes nodes along this main branch of the cluster. To zeroth order in $g_{+}$, this is simply $p_{e}^{q} /\left(n_{e} / n\right)^{\sigma}=1$, so that $\mathcal{Z}-1=O(\delta)$, and to zeroth order in $\delta,\langle\log \mathcal{Z}\rangle=0$.

\section{First order in $\delta$}

To first order in $\delta$, we can approximate

$$
\langle\log \mathcal{Z}\rangle \approx\langle\mathcal{Z}-1\rangle
$$

because the other terms in the expansion of the logarithm are at least of $O\left(\delta^{2}\right)$. The terms with only one factor of $f_{-}$represent elementary branches removed at some point from the main branch by the choice of the weaker branch at only one node. If there are $J$ nodes in the main branch, there are $J$ such "first-order" sidebranches. Since we are interested in terms of first order in $\delta$ only, for these terms the factors of $f_{+}$appearing in the partition function can be replaced by 1 , because $f_{+}=1+g_{+}=1+O(\delta)$. Thus the term of $O(\delta)$ that is proportional to $f_{-}$is 


$$
\langle\log Z\rangle_{1, \propto f_{-}}=\sum_{j \geq 1}\left\langle\frac{\rho_{0}}{n_{j}^{\nu}}\right\rangle\left\langle\left\langle f_{-}\right\rangle\right\rangle,
$$

where the subscript on the left-hand side represents terms of first order in $\delta$ that are also proportional to $\left\langle\left\langle f_{-}\right\rangle\right\rangle$. The index $j$ represents nodes on the main branch of the cluster, starting at the seed (or root) $j=1$.

There are also terms of first order in $\delta$ that are proportional to $\left\langle\left\langle g_{+}\right\rangle\right\rangle$. These terms include no factors of $f_{-}$, and refer to the elementary sub-branch at the end of the main branch. The actual partition sum contribution from this sub-branch is represented as a product of factors of $f_{+}$, one at each node. To first order in $\delta$, one chooses one of these nodes to contribute a factor of $g_{+}$, and all others contribute a factor of 1 . Thus

$$
\langle\log Z\rangle_{1, \propto g_{+}}=\sum_{j \geq 1}\left\langle\frac{\rho_{0}}{n_{j}^{\nu}}\right\rangle\left\langle\left\langle g_{+}\right\rangle\right\rangle
$$

where, again, the index $j$ refers to nodes on the main branch of the cluster.

Although the term $\propto g_{+}$is thus identical in form to that $\propto f_{-}$, Eq. (2.14), the origin of the two terms is quite different. Equation (2.14) gives the $O(\delta)$ partition sum contribution of all of the elementary sub-branches along the "main line" of the sidebranches of the main branch, while Eq. (2.15) gives the $O(\delta)$ contribution from the elementary sub-branch at the end of the main branch itself (Figure 7).

We can also represent these terms graphically. We represent a $g_{+}$"vertex" by an open circle, and an $f_{-}$vertex by a solid circle. Their sum

$$
\psi \equiv f_{-}+g_{+}
$$

(from Eq. (1.15)) is indicated by two circles connected by a short vertical line. A set of nodes on a branch over which $n_{j}^{-\nu}$ (and later more complicated functions of $n_{j}$ ) is summed is indicated by a solid horizontal line. The left side of a diagram indicates the root, and the right hand side represents structure successively further down the branching tree. Thus the $\langle\log \mathcal{Z}\rangle_{1}$ terms that we have written in Eqs. (2.14-15) above are indicated by the diagrams in Figure 8. 
The perturbation expansion we are developing is analogous to field theoretic perturbation series, with the factors of $f_{-}$and $g_{+}$playing the role of vertices, and the sums of $n^{-\nu}$ playing the role of propagators. We shall see below that at higher order, both of these objects become more complicated. One difference with field theory is that topologically, the diagrams always have the shape of branched trees, with no loops in the structure.

To evaluate these terms, we must evaluate $\left\langle\sum_{j} n_{j}^{-\nu}\right\rangle$, where the sum is along the nodes of the main branch. We shall evaluate this sum by a somewhat roundabout path. Consider the quantity $\log \left(n / n_{e}\right)$, where $n_{e}$ is the number of particles in the elementary branch at the end of the main branch. By the definition of the $y$ parameters (Eq. (1.10)), we can write the identity

$$
\log \left(n / n_{e}\right)=-\log \prod_{j=1}^{J}\left(1-y\left(\epsilon_{j} n_{j}\right)\right)
$$

where the index $j$ ranges over all of the nodes on the main branch, of which $J$ is the last. Taking the expectation value of the right-hand side, and using the methods we have developed above for computing these expectation values, we see (referring to Eqs. (2.7-8)) that

$$
\log n-\log n_{e}=-\sum_{j=1}^{J}\left\langle\frac{\rho_{0}}{n_{j}^{\nu}}\right\rangle\langle\langle\log (1-y)\rangle\rangle,
$$

so that

$$
\sum_{j=1}^{J}\left\langle\frac{\rho_{0}}{n_{j}^{\nu}}\right\rangle=\lambda \log n+a_{0}
$$

with $\lambda=-\langle\langle\log (1-y)\rangle\rangle^{-1}$ and $a_{0}=\log n_{e} /\langle\log (1-y)\rangle$. The parameter $\lambda$ is thus a function only of the unstable manifold in the $x-y$ plane. This is not the case with $a_{0}$, which is cutoff dependent, and thus sensitive to small-scale details of the theory. We shall see below that such non-universal constants do not affect results for multifractal dimensions. 
Thus our final result is that

$$
\langle\log \mathcal{Z}\rangle_{1}=\left\langle\left\langle f_{-}+g_{+}\right\rangle\right\rangle\left(\lambda \log n+a_{0}\right)
$$

We should remark that the non-universal constant $\rho_{0}$, and the exponent $\nu$, no longer appear explicitly in our formulae, although they do appear in intermediate steps in this computational method, and $\nu$ appears in the definition of the average $\langle\langle\cdots\rangle\rangle$. These quantities do not appear explicitly in final results at any order in $\delta$.

In Eqs. (1.15) and (2.16), we defined the quantity $f_{-}+f_{+}-1=f_{-}+g_{+} \equiv \psi$, and stated that the criterion $\langle\langle\psi\rangle\rangle=0$ determined the annealed multifractal dimensions $\sigma_{\mathcal{A}}(q)$. (Recall that $\psi$, like $f_{-}$and $g_{+}$, is a function of $q$ and $\sigma$.) Thus, in the limit $n \rightarrow \infty$, our $O(\delta)$ result for the quenched multifractal dimensions, determined by the criterion $\langle\log \mathcal{Z}\rangle_{1}=0$, is identical to our result for the annealed multifractal dimensions, so that $\sigma_{\mathcal{Q}}(q)=\sigma_{\mathcal{A}}(q)+O\left(\delta^{2}\right)$.

\section{Second order in $\delta$}

Although similar in spirit to the computation of $\langle\log \mathcal{Z}\rangle_{1}$, the computation of $\langle\log \mathcal{Z}\rangle_{2}$ introduces some new complications. Let us start by considering the term

arising from $\langle\mathcal{Z}-1\rangle_{2, \propto g_{+}^{2}}$. This term arises from the elementary sub-branch at the end of the main branch; in this case we are computing the $O\left(\delta^{2}\right)$ term in its contribution to the partition sum.

Specifically, we must compute

$$
\langle\mathcal{Z}-1\rangle_{2, \propto g_{+}^{2}}=\left\langle\sum_{k \geq 1} \sum_{j>k} g_{+}\left(\epsilon_{k} n_{k}\right) g_{+}\left(\epsilon_{j} n_{j}\right)\right\rangle
$$

Graphically, this term is represented by a horizontal bar interrupted by two open circles, representing the two factors of $g_{+}$, one downstream from the other on the main branch. Note the presence of two horizontal segments, corresponding to the two sums in Eq. (2.21) (Figure 9).

In Eq. (2.21), we start by averaging over $\epsilon_{j}$, thereby obtaining 


$$
\left\langle\sum_{k \geq 1} \sum_{j>k} g_{+}\left(\epsilon_{k} n_{k}\right) g_{+}\left(\epsilon_{j} n_{j}\right)\right\rangle=\left\langle\sum_{k \geq 1} \sum_{j>k} g_{+}\left(\epsilon_{k} n_{k}\right) \frac{\rho_{0}}{n_{j}^{\nu}}\right\rangle\left\langle\left\langle g_{+}\right\rangle\right\rangle
$$

Using the result from Eq. (2.19) for $\sum_{j \geq 1} n_{j}^{-\nu}$, we see that

$$
\left\langle\sum_{j>k} \frac{\rho_{0}}{n_{j}^{\nu}}\right\rangle=\left\langle\lambda \log n_{k+1}+a_{0}\right\rangle=\left\langle\lambda\left[\log n_{k}+\log \left(1-y\left(\epsilon_{k} n_{k}\right)\right)\right]+a_{0}\right\rangle .
$$

where $n_{k+1}$ refers to the number of particles in the stronger branch at the $k^{\prime} t h$ node on the main branch. Then, by summing over $j$ and averaging over $\epsilon_{k}$, we obtain

$$
\left.\langle\mathcal{Z}-1\rangle_{2, \propto g_{+}^{2}}=\left\langle\sum_{k \geq 1} \frac{\rho_{0}}{n_{k}^{\nu}}\left\{\lambda\left[\left\langle g_{+}\right\rangle\right\rangle \log n_{k}+\left\langle\left\langle g_{+} \log (1-y)\right\rangle\right\rangle\right]+a_{0}\left\langle\left\langle g_{+}\right\rangle\right\rangle\right\}\right\rangle\left\langle\left\langle g_{+}\right\rangle\right\rangle
$$

In order to perform the final sum, we must compute $\left\langle\sum_{k} \log n_{k} / n_{k}^{\nu}\right\rangle$. This can be done by an iterative procedure based upon our result above for $\sum_{j} n_{j}^{-\nu}$, Eq. (2.19) (details are given in appendix A.) The result is

$$
\left\langle\sum_{j \geq 1} \frac{\rho_{0} \log n_{j}}{n_{j}^{\nu}}\right\rangle=\left(\lambda_{2,2} \log ^{2} n+\lambda_{2,1} \log n+\lambda_{2,0}\right)
$$

where $n \equiv n_{1}$ is again the total number of particles in the entire cluster, and $\lambda_{2,0}$ is a non-universal constant. By contrast, $\lambda_{2,2}$ and $\lambda_{2,1}$ are functions only of the form of the unstable manifold. The generalization of Eq. (2.25) is straightforward

$$
\left\langle\sum_{j \geq 1} \frac{\rho_{0} \log ^{N-1} n_{j}}{n_{j}^{\nu}}\right\rangle=\sum_{M=0}^{N} \lambda_{N, M} \log ^{M} n
$$

In this notation, all of the $\left\{\lambda_{N, 0}\right\}$ are non-universal; the first of these is $\lambda_{1,0} \equiv a_{0}$. Furthermore, $\lambda \equiv \lambda_{1,1}$. In appendix A, an iterative method which can be used to compute arbitrary numbers of these coefficients (except, of course, for the non-universal ones) is demonstrated. For the purposes of this work, we need only $\lambda=\left\langle\langle\log (1-y\rangle\rangle^{-1}\right.$, $\lambda_{2,2}=\lambda / 2$, and $\lambda_{2,1}$, which is given by 


$$
\lambda_{2,1}=\frac{\left\langle\left\langle\log ^{2}(1-y)\right\rangle\right\rangle}{2\langle\langle\log (1-y)\rangle\rangle^{2}} \equiv \lambda^{\prime}
$$

Applying these results to the computation of the right-hand side of Eq. (2.24), we obtain

$$
\begin{aligned}
\langle\mathcal{Z}-1\rangle_{2, \propto g_{+}^{2}}= & \left(\lambda_{2,2} \log ^{2} n+\lambda_{2,1} \log n+\lambda_{2,0}\right) \lambda\left\langle\left\langle g_{+}\right\rangle\right\rangle^{2} \\
& +\left(\lambda \log n+a_{0}\right) \lambda\left\langle\left\langle g_{+} \log (1-y)\right\rangle\right\rangle\left\langle\left\langle g_{+}\right\rangle\right\rangle . \\
+ & \left(\lambda \log n+a_{0}\right) a_{0}\left\langle\left\langle g_{+}\right\rangle\right\rangle^{2}
\end{aligned}
$$

Another term, $\langle\mathcal{Z}-1\rangle_{2, \propto f_{-}^{2}}$, corresponds to partition sum contributions from elementary sub-branches with two weak ancestor nodes. In this case, since we wish to consider only terms of $O\left(\delta^{2}\right)$, we take no factors of $g_{+}$whatsoever. The diagrammatic representation of this term is shown in Figure 10. A short vertical bar is added before the second horizontal line, to indicate that one of the summations of $n^{-\nu}$ takes place off of the main branch. The calculation is entirely analogous to that leading to Eq. (2.28), and the result is

$$
\begin{aligned}
\langle\mathcal{Z}-1\rangle_{2, \propto f_{-}^{2}}= & \left(\lambda_{2,2} \log ^{2} n+\lambda_{2,1} \log n+\lambda_{2,0}\right) \lambda\left\langle\left\langle f_{-}\right\rangle\right\rangle^{2} \\
& +\left(\lambda \log n+a_{0}\right) \lambda\left\langle\left\langle f_{-} \log y\right\rangle\right\rangle\left\langle\left\langle f_{-}\right\rangle\right\rangle . \\
& +\left(\lambda \log n+a_{0}\right) a_{0}\left\langle\left\langle f_{-}\right\rangle\right\rangle^{2}
\end{aligned}
$$

The mixed terms (Figure 11), which contain one factor of $g_{+}$, and one factor of $f_{-}$(in either order), have the same structure,

$$
\begin{aligned}
\langle\mathcal{Z}-1\rangle_{2, \propto f_{-} \cdot g_{+}=} & \left.\left(\lambda_{2,2} \log ^{2} n+\lambda_{2,1} \log n+\lambda_{2,0}\right) 2 \lambda\left\langle\left\langle f_{-}\right\rangle\right\rangle\left\langle g_{+}\right\rangle\right\rangle \\
& +\left(\lambda \log n+a_{0}\right) \lambda\left[\left\langle\left\langle f_{-} \log y\right\rangle\right\rangle\left\langle\left\langle g_{+}\right\rangle\right\rangle+\left\langle\left\langle g_{+} \log (1-y)\right\rangle\right\rangle\left\langle\left\langle f_{-}\right\rangle\right\rangle\right] . \\
& +\left(\lambda \log n+a_{0}\right) 2 a_{0}\left\langle\left\langle f_{-}\right\rangle\right\rangle\left\langle\left\langle g_{+}\right\rangle\right\rangle
\end{aligned}
$$

There are also contributions to $\langle\log \mathcal{Z}\rangle_{2}$ from the $-(1 / 2)\left\langle(\mathcal{Z}-1)^{2}\right\rangle$ term in the expansion of the logarithm. These reflect cross-products between two elementary subbranches, with the partition sum contribution of each being taken to $O(\delta)$. The di- 
agrams appearing are shown in Figure 12. Note that symmetry factors appear multiplying some of the diagrams. Also, new vertices of the type $\left\langle\left\langle g_{+}^{2}\right\rangle\right\rangle,\left\langle\left\langle f_{-}^{2}\right\rangle\right\rangle$ appear, arising from cases in which $g_{+}$or $f_{-}$factors are taken at the same vertices in the two different "replicas" of $\mathcal{Z}-1$. Such vertices are indicated by adjoining circles, displaced perpendicularly. The contribution $\propto g_{+}^{2}$ is

$$
\begin{aligned}
-\frac{1}{2}\left\langle(\mathcal{Z}-1)^{2}\right\rangle_{2, \propto g_{+}^{2}}=- & \left(\lambda_{2,2} \log ^{2} n+\lambda_{2,1} \log n+\lambda_{2,0}\right) \lambda\left\langle\left\langle g_{+}\right\rangle\right\rangle^{2} \\
& -\left(\lambda \log n+a_{0}\right) \lambda\left\langle\left\langle g_{+} \log (1-y)\right\rangle\right\rangle\left\langle\left\langle g_{+}\right\rangle\right\rangle \\
- & \left(\lambda \log n+a_{0}\right) a_{0}\left\langle\left\langle g_{+}\right\rangle\right\rangle^{2} \\
& -\frac{1}{2}\left(\lambda \log n+a_{0}\right)\left\langle\left\langle g_{+}^{2}\right\rangle\right\rangle,
\end{aligned}
$$

while that $\propto f_{-}^{2}$ is

$$
\begin{aligned}
-\frac{1}{2}\left\langle(\mathcal{Z}-1)^{2}\right\rangle_{2, \propto f_{-}^{2}}=- & \left(\lambda_{2,2} \log ^{2} n+\lambda_{2,1} \log n+\lambda_{2,0}\right) \lambda\left\langle\left\langle f_{-}\right\rangle\right\rangle^{2} \\
& \left.-\left(\lambda \log n+a_{0}\right) \lambda\left\langle\left\langle f_{-} \log (1-y)\right\rangle\right\rangle\left\langle f_{-}\right\rangle\right\rangle \\
- & \left(\lambda \log n+a_{0}\right) a_{0}\left\langle\left\langle f_{-}\right\rangle\right\rangle^{2} \\
& -\frac{1}{2}\left(\lambda \log n+a_{0}\right)\left\langle\left\langle f_{-}^{2}\right\rangle\right\rangle .
\end{aligned}
$$

Note that the vertex $\left\langle\left\langle f_{-} \log (1-y)\right\rangle\right\rangle$ appears in this term, rather than $\left\langle\left\langle f_{-} \log y\right\rangle\right\rangle$, as in Eq. (2.29). Finally, the mixed term is

$$
\begin{aligned}
-\frac{1}{2}\left\langle(\mathcal{Z}-1)^{2}\right\rangle_{2, \propto f_{-} \cdot g_{+}}=- & \left(\lambda_{2,2} \log ^{2} n+\lambda_{2,1} \log n+\lambda_{2,0}\right) 2 \lambda\left\langle\left\langle f_{-}\right\rangle\right\rangle\left\langle\left\langle g_{+}\right\rangle\right\rangle \\
- & \left(\lambda \log n+a_{0}\right)\left[\lambda\left\langle\left\langle f_{-} \log (1-y)\right\rangle\right\rangle\left\langle\left\langle g_{+}\right\rangle\right\rangle\right. \\
& \left.+\lambda\left\langle\left\langle f_{-}\right\rangle\right\rangle\left\langle\left\langle g_{+} \log (1-y)\right\rangle\right\rangle\right] \\
- & \left.\left(\lambda \log n+a_{0}\right) 2 a_{0}\left\langle\left\langle f_{-}\right\rangle\right\rangle\left\langle g_{+}\right\rangle\right\rangle \\
- & \left(\lambda \log n+a_{0}\right)\left\langle\left\langle f_{-} \cdot g_{+}\right\rangle\right\rangle .
\end{aligned}
$$

Collecting terms from Eqs. (2.28-33), we finally obtain 


$$
\langle\log \mathcal{Z}\rangle_{2}=\left[\lambda\langle\langle\psi\rangle\rangle\left\langle\left\langle f_{-} \log \left(\frac{y}{1-y}\right)\right\rangle\right\rangle-\frac{1}{2}\left\langle\left\langle\psi^{2}\right\rangle\right\rangle\right]\left(\lambda \log n+a_{0}\right)
$$

where, again, $\psi=f_{-}+g_{+}$. Note that the $\log ^{2} n$ term has cancelled out, as have the non-universal contributions to the $\log n$ term $\left(\propto a_{0} \lambda \log n\right.$ in the above Eqs. (2.28-33)).

\section{Structure of $\delta$ expansion}

In appendix $\mathrm{B}$, we continue this expansion, deriving $\langle\log \mathcal{Z}\rangle_{3}$ and $\langle\log \mathcal{Z}\rangle_{4}$. In general, we expect that the $O\left(\delta^{N}\right)$ term in the expansion will have the form

$$
\langle\log \mathcal{Z}\rangle_{N}=\sum_{M=0}^{N} \beta_{N, M} \log ^{M} n
$$

where the coefficients $\beta_{N, M}$ will include "universal" (independent of the $\left\{\lambda_{N, 0}\right\}$ of Eq. (2.26)) and non-universal terms. It is clear from the structure of the expansion that the leading coefficients $\beta_{N, N}$ do not contain any non-universal terms, as the leading coefficient $\lambda_{N, N}$ in Eq. (2.26) for any sum $\sum_{j} \log ^{N-1} n_{j} / n_{j}^{\nu}$ is universal (see Eq. (A.8) below). We also note that with the exception of the $O(\delta)$ term, we have $\beta_{N, N}=0$ to fourth order. Below we will show that this is true to all orders. The situation is summarized in Table I, which shows, as a function of order in $\delta$ and power of $\log n$, the terms appearing in this series.

Unfortunately, this series does not allow us to directly determine the quenched dimensions $\sigma_{\mathcal{Q}}(q)$. Recall that these are determined by the requirement that $\langle\log \mathcal{Z}\rangle \rightarrow 0$ in the limit $n \rightarrow \infty$ (more precisely, that it be bounded above and below by quantities which go to neither $\pm \infty$, see Ref. 20 for a discussion). Since each individual term $\log ^{M} n$ diverges as $n \rightarrow \infty$, and since there is no obvious relation between the coefficients of different powers of $\log n$ in the series for $\langle\log \mathcal{Z}\rangle$, we cannot extract unique results for $\sigma_{\mathcal{Q}}(q)$ from this series.

For this reason, we turn in section 3 to the resummation to all orders in $\delta$ of the leading and sub-leading divergent terms in this series. We shall see that the annoying higher order logarithms can be safely resummed to decaying power laws, allowing unambiguous determination of $\sigma_{\mathcal{Q}}(q)$ in the limit $n \rightarrow \infty$. 


\section{Resummation of "one-propagator" series}

Before turning to the general re-summation of the series, we would like to remark on a simpler resummation, which is analogous to loop expansions in ordinary field theory. Consider all terms in the series with only one summation over $n^{-\nu}$. These terms can be of any order in $\delta$, as vertices can be multiplied together at the same node, as in the $O\left(\delta^{2}\right)$ terms above. The associated diagrams are shown in Figure 13. This "one-propagator" result can be easily derived:

$$
\langle\log \mathcal{Z}\rangle_{1 p}=\sum_{N=1}^{\infty} \frac{(-1)^{N-1}}{N}\left\langle\left\langle\psi^{N}\right\rangle\right\rangle\left(\lambda \log n+a_{0}\right)
$$

We have used the subscript $1 p$ to indicate that this is the one-propagator term. Taking the sum inside the brackets $\langle\langle\rangle\rangle$ yields

$$
\langle\log \mathcal{Z}\rangle_{1 p}=\langle\langle\log (1+\psi)\rangle\rangle\left(\lambda \log n+a_{0}\right)
$$

This result has two appealing properties-in the first place it leads to a result for $\sigma_{\mathcal{Q}}(q)$ in which no negative values of $f$ appear. It is thus an appealing approximate formula for the dimensions of a "typical" cluster. Also, as we shall see in section 4, the $\sigma_{\mathcal{Q}}(q)$ resulting from setting $\langle\log \mathcal{Z}\rangle_{1 p}=0$ is quite close to numerical results in the case of our toy model, model Z.

However, if one tries to extend this approach by resumming the "two-propagator" terms, and so forth, one encounters the same problem as with the $\delta$-series, that higher orders in $\log n$ are also generated. Thus this expansion technique suffers from the same difficulty as the $\delta$ expansion, that unique values of $\sigma_{\mathcal{Q}}(q)$ are impossible to obtain without resummation.

\section{Resummation of the $\delta$-series}

We have shown that the form of the $\delta$-series is:

$$
\langle\log \mathcal{Z}\rangle=\sum_{N=1}^{\infty} \delta^{N}\langle\log \mathcal{Z}\rangle_{N}=\sum_{N=1}^{\infty} \sum_{M=0}^{N} \beta_{N, M} \log ^{M} n
$$


where each $\beta_{N, M}$ is of $O\left(\delta^{N}\right)$. Our procedure is now to resum this series by summing first the most divergent terms $M=N$ at all orders in $\delta$, then the second-most divergent terms $M=N-1$, and so on. Introducing yet another subscript, $\ell$ (leading), to indicate the most divergent sum, and the subscript $s \ell$ (sub-leading), to indicate the next most divergent sum, we have

$$
\langle\log \mathcal{Z}\rangle_{\ell}=\sum_{N=1}^{\infty} \beta_{N, N} \log ^{N} n
$$

and

$$
\langle\log \mathcal{Z}\rangle_{s \ell}=\sum_{N=2}^{\infty} \beta_{N, N-1} \log ^{N-1} n
$$

In principle, we could continue with sub-sub leading terms, but in practice we will restrict ourselves to computing only these two terms. Note that our resummation procedure corresponds to summing down the diagonals of Table I.

\section{Summation of leading terms}

The leading logarithm at each order in $\delta$ will be universal, as pointed out in section 2 above. Let us start by computing $\langle Z-1\rangle_{\ell}$. In order to compute this, we will need the result from appendix $\mathrm{A}: \lambda_{N, N}=\lambda / N$, where the reader might recall the definition of $\lambda_{N, M}$

$$
\left\langle\sum_{j \geq 1} \frac{\rho_{0} \log ^{N-1} n_{j}}{n_{j}^{\nu}}\right\rangle=\sum_{M=0}^{N} \lambda_{N, M} \log ^{M} n .
$$

Here the sum is along a horizontal bar of some diagram. Since we are taking the leading order in $\log n$ in the entire diagram, we wish to take the leading order in each propagator. No factors of $1-y$ or $y$ will appear in the leading order, since each such factor would take the place of a factor of $\log n$. Also, we take the leading logarithm of each term of the form of Eq. (3.4), which will have as a coefficient one of the $\left\{\lambda_{N, N}\right\}$.

Consider a diagram contributing to $\langle\mathcal{Z}-1\rangle_{\ell}$ consisting of $n_{1}$ factors of $f_{-}$and $n_{2}$ factors of $g_{+}$. There will be a total of $N=n_{1}+n_{2}$ horizontal bars, or propagator 
sums, in such a diagram (see Figure 14). If we call the contribution of this diagram $\mathcal{C}$, then

$$
\mathcal{C}=\frac{\lambda^{N}}{N !}\left\langle\left\langle f_{-}\right\rangle\right\rangle^{n_{1}}\left\langle\left\langle g_{+}\right\rangle\right\rangle^{n_{2}} \log ^{N} n
$$

because $\lambda^{N} / N !=\prod_{N^{\prime}=1}^{N} \lambda_{N^{\prime}, N^{\prime}}$.

Since any vertex can be taken to be either $f_{-}$or $g_{+}$without changing other features of the term, we immediately obtain

$$
\langle\mathcal{Z}-1\rangle_{\ell}=\sum_{N=1}^{\infty} \frac{\lambda^{N}}{N !}\langle\langle\psi\rangle\rangle^{N} \log ^{N} n=\exp (\lambda\langle\langle\psi\rangle\rangle \log n)-1
$$

Now let us turn to $-1 / 2\left\langle(\mathcal{Z}-1)^{2}\right\rangle_{\ell}$ from the expansion of $\langle\log \mathcal{Z}\rangle$ in Eq. (2.1). For the sake of argument, let us consider terms in which each factor of $\mathcal{Z}-1$ has at least one factor of $f_{-}$. We will say that the first factor of $\mathcal{Z}-1$ has $l_{1}$ factors of $g_{+}$before the first appearance of $f_{-}$, and $m_{1}$ factors of either $f_{-}$or $g_{+}$after the first appearance of $f_{-}$. The second factor of $\mathcal{Z}-1$ has $l_{2}$ factors of $g_{+}$before the first factor of $f_{-}$, and $m_{2}$ factors of either $f_{-}$or $g_{+}$after the first factor of $f_{-}$(see Figure 15).

Now we average these two factors together. Note that two factors of $f_{-}$appearing in the main line cannot appear at the same node; were they to be averaged together, the diagram overall would lose one propagator, and thus one power of $\log n$, without losing a factor of $\delta$, which counts the total number of factors of $f_{-}$and $g_{+}$. Such diagrams first contribute at sub-leading order. Similarly, two factors of $g_{+}$cannot appear at the same node.

Suppose that the factor of $f_{-}$coming from the second $\mathcal{Z}-1$ is further down the main line than that coming from the first $\mathcal{Z}-1$. Then in any diagram resulting from this product, there will be a number $l \leq l_{2}$ of $g_{+}$vertices between the two $f_{-}$ vertices, and $L-l$ to the left (further up the main branch) of the first $f_{-}$vertex, where $L=l_{1}+l_{2}$ (see Figure 16). Now we must consider the vertices off the main line. The structure of the diagram does not depend on whether these are $f_{-}$or $g_{+}$vertices, since we average no factors of $\log (y)$ or $\log (1-y)$ with these vertices at leading order. Thus 
we will sum together these two types of off-main line vertices, and attach $m_{1}$ vertices $\psi$ to the first factor of $f_{-}$, and $m_{2}$ such vertices to the second factor of $f_{-}$.

In order to compute the diagram, we must keep track of the number of factors of $\log n$ appearing for each propagator; at leading order we take only the propagator terms multiplying one of elements of the set $\left\{\lambda_{N, N}\right\}$. The total number of propagators to the right (on the main line) of the "upstream" factor of $f_{-}$is $m_{2}+l+1$, while the total number downstream of the first factor of $g_{+}$to the left of this $f_{-}$vertex is $m_{1}+m_{2}+l+2$. (Note that we use "upstream" to mean closer to the root, not closer to the elementary sub-branches.) A simple computation, keeping careful track of these factors, gives the contribution $\mathcal{W}_{l}$ of this particular diagram as:

$$
\begin{aligned}
& \mathcal{W}_{l}= \frac{1}{\left(m_{2}+l+1\right) !} \frac{1}{m_{1} !} \frac{(M+l+1) !}{(M+L+2) !} \\
& \cdot\left\langle\left\langle f_{-}\right\rangle\right\rangle^{2}\left\langle\left\langle g_{+}\right\rangle\right\rangle^{L}\langle\langle\psi\rangle\rangle^{M}(\lambda \log n)^{M+L+2}
\end{aligned}
$$

where $M=m_{1}+m_{2}$ and we defined above $L=l_{1}+l_{2}$.

Of course, the two original factors of $\mathcal{Z}-1$ can be multiplied together in various distinguishable ways to make this diagram, corresponding to the different origins of the $L-l$ factors of $g_{+}$on the left hand side of the diagram. Since $l_{1}$ of these come from the first factor of $\mathcal{Z}-1$, and $L-l-l_{1}$ come from the second factor of $\mathcal{Z}-1$, the total number of relative permutations $N_{p}$ is

$$
N_{p}=\left(\begin{array}{c}
L-l \\
l_{1}
\end{array}\right) \equiv \frac{(L-l) !}{\left(L-l-l_{1}\right) ! l_{1} !}
$$

Finally, to obtain the expectation value of the product of the factor $\mathcal{Z}-1$ containing $l_{1}$ factors of $g_{+}$in the main line and $m_{1}$ factors of $\psi$ off the main line with the other factor of $\mathcal{Z}-1$, which contains $l_{2}$ factors of $g_{+}$on the main line and $m_{2}$ factors of $\psi$ off the main line, we must sum over $l \leq l_{2}$ and permute the choice of whose main line factor of $f_{-}$is upstream (see Figure 16). If we are interested in contributions to $-(1 / 2)\left\langle(\mathcal{Z}-1)^{2}\right\rangle$ with at least two factors of $\left\langle\left\langle f_{-}\right\rangle\right\rangle$, we must then sum over $m_{1}, m_{2}$, $l_{1}$, and $l_{2}$. This yields 


$$
\begin{aligned}
-\frac{1}{2}\left\langle(\mathcal{Z}-1)^{2}\right\rangle_{\left.\ell, \propto\left\langle f_{-}\right\rangle\right\rangle^{2}}=- & \sum_{m_{1}=0}^{\infty} \sum_{m_{2}=0}^{\infty} \sum_{l_{1}=0}^{\infty} \sum_{l_{2}=0}^{\infty} \sum_{l=0}^{l_{2}} \\
& \cdot\left(\begin{array}{c}
L-l \\
l_{1}
\end{array}\right) \frac{1}{\left(m_{2}+l+1\right) !} \frac{1}{m_{1} !} \frac{(M+l+1) !}{(M+L+2) !} \\
\cdot & \left\langle\left\langle f_{-}\right\rangle\right\rangle^{2}\left\langle\left\langle g_{+}\right\rangle\right\rangle^{L}\langle\langle\psi\rangle\rangle^{M}(\lambda \log n)^{M+L+2} .
\end{aligned}
$$

In addition, the term including only one factor of $f_{-}$is easily written as

$$
\begin{aligned}
-\frac{1}{2}\left\langle(\mathcal{Z}-1)^{2}\right\rangle_{\ell, \propto\left\langle f_{-}\right\rangle}=- & \sum_{m_{1}=0}^{\infty} \sum_{l_{1}=0}^{\infty} \sum_{l_{2}=1}^{\infty} \sum_{l=0}^{l_{2}} \\
& \cdot\left(\begin{array}{c}
L-l \\
l_{1}
\end{array}\right) \frac{1}{m_{1} !} \frac{1}{l !} \frac{\left(m_{1}+l\right) !}{\left(m_{1}+L+1\right) !} \\
\cdot & \left\langle\left\langle f_{-}\right\rangle\right\rangle\left\langle\left\langle g_{+}\right\rangle\right\rangle^{L}\langle\langle\psi\rangle\rangle^{m_{1}}(\lambda \log n)^{m_{1}+L+1},
\end{aligned}
$$

while that with no factors of $f_{-}$at all is

$$
-\frac{1}{2}\left\langle(\mathcal{Z}-1)^{2}\right\rangle_{\ell, \propto\left\langle\left\langle f_{-}\right\rangle\right\rangle^{0}}=-\frac{1}{2} \sum_{l_{1}=1}^{\infty} \sum_{l_{2}=1}^{\infty}\left(\begin{array}{c}
L \\
l_{1}
\end{array}\right) \frac{1}{L !}\left\langle\left\langle g_{+}\right\rangle\right\rangle^{L}(\lambda \log n)^{L}
$$

The sums are tedious but elementary. In appendix $\mathrm{C}$ we illustrate the performance of the types of sums appearing in this work. Performing the sums and adding Eqs. (3.911) we obtain the quite simple result,

$$
-\frac{1}{2}\left\langle(\mathcal{Z}-1)^{2}\right\rangle_{\ell}=-\frac{1}{2}[\exp (\lambda\langle\langle\psi\rangle\rangle \log n)-1]^{2}=-\frac{1}{2}\left[\langle\mathcal{Z}-1\rangle_{\ell}\right]^{2}
$$

Remarkably, at leading order, the process of taking the expectation value of $\mathcal{Z}-1$ commutes with taking the square of this quantity. We will now address the origin of this identity.

Let us first consider a simpler case, with only one factor of $\mathcal{Z}-1$. The leading order $\mathcal{D}$ of a diagram with $l$ factors of $g_{+}$on the main line, followed by one factor of $f_{-}$, followed by $m$ factors of $\psi$, can be written as 


$$
\mathcal{D}=\mathcal{N}\left(\lambda\left\langle\left\langle f_{-}\right\rangle\right\rangle \log n\right)\left(\lambda\left\langle\left\langle g_{+}\right\rangle\right\rangle \log n\right)^{l}(\lambda\langle\langle\psi\rangle\rangle \log n)^{m}
$$

where the factor $\mathcal{N}=1 /(m+l+1)$ !. The factor $\mathcal{N}$ can be written as a nested integral over $l+1$ variables $x_{i}$ :

$$
\mathcal{N}=\left(\prod_{i=1}^{l} \int_{0}^{x_{i-1}} d x_{i}\right) \int_{0}^{x_{l}} d x_{l+1} \frac{x_{l+1}^{m}}{m !}=\frac{1}{(m+l+1) !}
$$

with $x_{0} \equiv 1$. In this product, the $l$ integrals on the left correspond to $g_{+}$vertices, while the last integral corresponds to the $f_{-}$factor and the accompanying insertion of $m$ additional factors of $\log n$ associated with the $\psi$ vertices.

Now consider a term arising from the product of a factor of $\mathcal{Z}-1$ containing $l_{1}$ factors of $g_{+}$in the main line and $m_{1}$ factors of $\psi$ off the main line with another factor of $\mathcal{Z}-1$ containing $l_{2}$ factors of $g_{+}$and $m_{2}$ factors of $\psi$. Again, we choose $L=l_{1}+l_{2}$ and $M=m_{1}+m_{2}$. Each factor of $\mathcal{Z}-1$ in addition has one $f_{-}$vertex on the main line. At leading order, any diagram $\mathcal{D}$ resulting from this product will have the form

$$
\mathcal{D}=\mathcal{N}\left(\lambda\left\langle\left\langle f_{-}\right\rangle\right\rangle \log n\right)^{2}\left(\lambda\left\langle\left\langle g_{+}\right\rangle\right\rangle \log n\right)^{L}(\lambda\langle\langle\psi\rangle\rangle \log n)^{M}
$$

where the combinatorial factor $\mathcal{N}$ depends on the precise way in which the diagrams are multiplied together. It is simple to write a formula for $\mathcal{N}$. Let us assign variables $x_{i}^{(1)}$ to the main line vertices arising from the first factor of $\mathcal{Z}-1$, and variables $x_{j}^{(2)}$ to main line vertices arising from the second factor of $\mathcal{Z}-1$. Then the factor $\mathcal{N}$ for any particular diagram can be written analogously to Eq. (3.14). For instance, if all of the factors of $g_{+}$coming from the second factor of $\mathcal{Z}-1$ are further down the main line (to the right of) the factor of $f_{-}$arising from the first factor of $\mathcal{Z}-1$, then we immediately see that 


$$
\begin{aligned}
\mathcal{N}= & \left(\prod_{i=1}^{l_{1}} \int_{0}^{x_{i-1}^{(1)}} d x_{i}^{(1)}\right) \int_{0}^{x_{l_{1}}^{(1)}} d x_{l_{1}+1}^{(1)} \frac{\left(x_{l_{1}+1}^{(1)}\right)^{m_{1}}}{m_{1} !} \\
& \cdot\left(\prod_{j=1}^{l_{2}} \int_{0}^{x_{j-1}^{(2)}} d x_{j}^{(2)}\right) \int_{0}^{x_{l_{2}}} d x_{l_{2}+1} \frac{\left(x_{l_{2}+1}^{(2)}\right)^{m_{2}}}{m_{2} !} \\
= & \frac{\left(M+l_{2}+1\right) !}{(M+L+2) !} \frac{1}{m_{1} !} \frac{1}{\left(m_{2}+l_{2}+1\right) !}
\end{aligned}
$$

with $x_{0}^{(2)}=x_{l_{1}+1}^{(1)}$ and $x_{0}^{(1)}=1$. Now the allowed permutations of the vertices correspond to all permutations of orderings of $\left\{x_{i}^{(1)}\right\},\left\{x_{j}^{(2)}\right\}$ within the multiple integral that preserve the orderings $1>x_{1}^{(1)}>x_{2}^{(1)} \cdots$ and $1>x_{1}^{(2)}>x_{2}^{(2)} \ldots$. But this allows one to factor the two nested integrals over the points $\left\{x^{(1)}\right\}$ and $\left\{x^{(2)}\right\}$ completely, so that the sum over permutations $P$ of $\mathcal{N}$ can be expressed as

$$
\begin{aligned}
\sum_{P} \mathcal{N}= & \left(\prod_{i=1}^{l_{1}} \int_{0}^{x_{i-1}^{(1)}} d x_{i}^{(1)}\right) \int_{0}^{x_{l_{1}}^{(1)}} d x_{l_{1}+1}^{(1)} \frac{\left(x_{l_{1}+1}^{(1)}\right)^{m_{1}}}{m_{1} !} \\
& \cdot\left(\prod_{j=1}^{l_{2}} \int_{0}^{x_{j-1}^{(2)}} d x_{j}^{(2)}\right) \int_{0}^{x_{l_{2}}^{(2)}} d x_{l_{2}+1}^{(2)} \frac{\left(x_{l_{2}+1}^{(2)}\right)^{m_{2}}}{m_{2} !} \\
= & \frac{1}{\left(m_{1}+l_{1}+1\right) !} \frac{1}{\left(m_{2}+l_{2}+1\right) !}
\end{aligned}
$$

where $x_{0}^{(2)}=1$, which deconvolves the two nested integrals, allowing their factorization into terms of the form (3.14). This provides a direct proof of the identity (3.12).

Using the same argument, we can easily show that at leading order,

$$
\left\langle(\mathcal{Z}-1)^{p}\right\rangle_{\ell}=\left[\langle\mathcal{Z}-1\rangle_{\ell}\right]^{p}=[\exp (\lambda\langle\langle\psi\rangle\rangle \log n)-1]^{p}
$$

so that the leading order contribution to $\langle\log \mathcal{Z}\rangle$ is

$$
\begin{aligned}
\langle\log \mathcal{Z}\rangle_{\ell} & =\sum_{p=1}^{\infty} \frac{(-1)^{p-1}}{p}\left(\langle\mathcal{Z}-1\rangle_{\ell}\right)^{p} \\
& =\lambda\langle\langle\psi\rangle\rangle \log n,
\end{aligned}
$$


We therefore arrive at the result

$$
\langle\log \mathcal{Z}\rangle_{\ell}=\log \left(1+\langle Z-1\rangle_{\ell}\right)
$$

We shall see that this is true also at sub-leading order, provided we restrict ourselves to terms diverging logarithmically with $n$.

\section{Summation of sub-leading terms: Universal}

Sub-leading terms are those with one less power of $\log n$ than $\delta$. There are two ways in which such terms can arise:

1. In a term arising from $\left\langle(\mathcal{Z}-1)^{p}\right\rangle$ with $p>1$, more than one factor of $f_{-}$or $g_{+}$ may appear at the same vertex. Since these two or more factors are integrated together, the net result is that the order of the term in $\log n$ is reduced with respect to the order in $\delta$. If more than two factors of $f_{-}$or $g_{+}$appear at the same vertex, or if more than one vertex contains more than one factor, then at least two factors of $\log n$ will be lost with respect to factors of $\delta$, so the term will be at most of sub-sub-leading order. Thus we need only consider diagrams with one vertex possessing two factors of $f_{-}$or $g_{+}$, with all other vertices containing at most one factor.

2. Recall that the form of the propagator sum along a particular sub-branch up to a given vertex is $\sum_{M=0}^{N} \lambda_{N, M} \log ^{M} n^{\prime}$, where $n^{\prime}$ is the number of particles below the vertex in question, either on the weak or the strong side (See Eq. (2.26)). Thus either $n^{\prime}=y(\epsilon n) n$, or $n^{\prime}=(1-y(\epsilon n)) n$, where $\epsilon$ is the random variable at the vertex being explicitly considered, and $n$ is the total number of descendants below that vertex. There are two ways in which the order of the propagator in $\log n$ may be reduced by one. The first way is for a factor of $\log y \operatorname{or} \log (1-y)$ to be taken in the highest order in $\log n^{\prime}$ term $(M=N)$ in the propagator. The second way is for the second highest term in $\log n^{\prime}(M=N-1)$ to be taken, but without including any factors of $\log (1-y)$ or $\log y$.

Terms of type 1

An example of a term of type 1. is provided by $\langle\log \mathcal{Z}\rangle_{s \ell, \propto\left\langle f_{-}^{2}\right\rangle}$. There is no 
contribution from $\langle\mathcal{Z}-1\rangle$ to this term; the first contribution arises from $-(1 / 2)\langle(\mathcal{Z}-$ $\left.1)^{2}\right\rangle$ (see Figure 17). It is easy to see that

$$
\begin{array}{r}
\left\langle(\mathcal{Z}-1)^{2}\right\rangle_{s \ell, \propto\left\langle\left\langle f_{-}^{2}\right\rangle\right.}=\sum_{l_{1}=0}^{\infty} \sum_{l_{2}=0}^{\infty} \sum_{m_{1}=0}^{\infty} \sum_{m_{2}=0}^{\infty}\left(\begin{array}{c}
L \\
l_{1}
\end{array}\right) \frac{1}{m_{1} !} \frac{1}{m_{2} !} \frac{M !}{(M+L+1) !} \\
\cdot\left(\lambda\left\langle\left\langle g_{+}\right\rangle\right\rangle \log n\right)^{L}(\lambda\langle\langle\psi\rangle\rangle \log n)^{M}\left(\lambda\left\langle\left\langle f_{-}^{2}\right\rangle\right\rangle \log n\right) .
\end{array}
$$

As in the above, $M=m_{1}+m_{2}$, and $L=l_{1}+l_{2}$. The sums are straightforward, and yield

$$
\left\langle(\mathcal{Z}-1)^{2}\right\rangle_{s \ell, \propto\left\langle f_{-}^{2}\right\rangle}=\frac{\left\langle\left\langle f_{-}^{2}\right\rangle\right\rangle}{2\left\langle\left\langle f_{-}\right\rangle\right\rangle}\left[\exp (2 \lambda\langle\langle\psi\rangle\rangle \log n)-\exp \left(2 \lambda\left\langle\left\langle g_{+}\right\rangle\right\rangle \log n\right)\right]
$$

The sub-leading terms proportional to $\left\langle\left\langle g_{+}^{2}\right\rangle\right\rangle$ and $\left\langle\left\langle f_{-} g_{+}\right\rangle\right\rangle$are computed in exactly the same way, and combine with the term proportional to $\left\langle\left\langle f_{-}^{2}\right\rangle\right\rangle$ to yield

$$
\left\langle(\mathcal{Z}-1)^{2}\right\rangle_{s \ell, \propto\left\langle\left\langle\psi^{2}\right\rangle\right\rangle}=\frac{\left\langle\left\langle\psi^{2}\right\rangle\right\rangle}{2\left\langle\left\langle f_{-}\right\rangle\right\rangle}\left[\exp (2 \lambda\langle\langle\psi\rangle\rangle \log n)-\exp \left(2 \lambda\left\langle\left\langle g_{+}\right\rangle\right\rangle \log n\right)\right] .
$$

Now we must compute the sub-leading contribution $\propto\left\langle\left\langle\psi^{2}\right\rangle\right\rangle$ arising from $\langle(\mathcal{Z}-$ $\left.1)^{p}\right\rangle$, with $p>2$. A typical diagram is shown in Figure 18. There are a total of $p(p-1) / 2$ choices of the two factors of $\mathcal{Z}-1$ which contribute the factors of $f_{-}$, $g_{+}$which will be integrated together. The computation is considerably simplified by the fact that the remaining factors of $\mathcal{Z}-1$ can be averaged separately of these two factors, by a simple extension of identity (3.18), which we used above to factor the leading order terms. Thus

$$
\left\langle(\mathcal{Z}-1)^{p}\right\rangle_{s \ell, \propto\left\langle\left\langle\psi^{2}\right\rangle\right.}=\frac{p(p-1)}{2}\left\langle(\mathcal{Z}-1)^{2}\right\rangle_{s \ell, \propto\left\langle\left\langle\psi^{2}\right\rangle\right.}\left[\langle\mathcal{Z}-1\rangle_{\ell}\right]^{p-2},
$$

leading to 


$$
\begin{aligned}
\langle\log \mathcal{Z}\rangle_{s \ell, \propto\left\langle\left\langle\psi^{2}\right\rangle\right\rangle} & =\left\langle(\mathcal{Z}-1)^{2}\right\rangle_{s \ell, \propto\left\langle\left\langle\psi^{2}\right\rangle\right.} \sum_{p=2}^{\infty} \frac{(-1)^{p-1}}{p} \frac{p(p-1)}{2}\left[\langle\mathcal{Z}-1\rangle_{\ell}\right]^{p-2} \\
& =-\left\langle(\mathcal{Z}-1)^{2}\right\rangle_{s \ell, \propto\left\langle\left\langle\psi^{2}\right\rangle\right.} \frac{1}{2}\left(\frac{1}{1+\langle\mathcal{Z}-1\rangle_{\ell}}\right)^{2} .
\end{aligned}
$$

Using Eqs. (3.6) and (3.23), and substituting from Eq. (3.25), we then obtain

$$
\begin{aligned}
\langle\log \mathcal{Z}\rangle_{\left.s \ell, \propto\left\langle\psi^{2}\right\rangle\right\rangle} & =-\frac{\left\langle\left\langle\psi^{2}\right\rangle\right\rangle}{4\left\langle\left\langle f_{-}\right\rangle\right\rangle}\left[1-\exp \left(-2 \lambda\left\langle\left\langle f_{-}\right\rangle\right\rangle \log n\right)\right] \\
& =-\frac{1}{4} \frac{\left\langle\left\langle\psi^{2}\right\rangle\right\rangle}{\left\langle\left\langle f_{-}\right\rangle\right\rangle}\left(1-n^{-2 \lambda\left\langle\left\langle f_{-}\right\rangle\right.}\right)
\end{aligned}
$$

Recall the definition of $\left\langle\left\langle f_{-}\right\rangle\right\rangle$:

$$
\left\langle\left\langle f_{-}\right\rangle\right\rangle \equiv \int_{0}^{\infty} d \eta \eta^{\nu-1} \frac{x^{q}(\eta)}{y^{\sigma}(\eta)}
$$

where $x(\eta)$ and $y(\eta)$ refer to the unstable manifold for branch competition. Clearly $\left\langle\left\langle f_{-}\right\rangle\right\rangle>0$, which implies that the correction to $\langle\log \mathcal{Z}\rangle$ displayed in Eq. (3.26) approaches a constant as $n \rightarrow \infty$. Thus this correction will not affect the values of the multifractal exponents $\sigma_{\mathcal{Q}}(q)$ (defined in Eq. (1.6)), provided that they are computed in this limit, as the terms $\operatorname{logarithmic}$ in $n$ in $\langle\log \mathcal{Z}\rangle$ will still dominate.

\section{Terms of type 2}

Now we turn to sub-leading terms of type 2, for which a sub-dominant term in one of the propagators is taken. We first consider such terms involving factors of $\log (1-y)$ or $\log y$ (to be averaged with $f_{-}$or $g_{+}$) arising from the expansion of a leading propagator term,

$$
\lambda_{N, N}\{\log [(1-y) n]\}^{N}=\lambda_{N, N}\left[\log ^{N} n+N \log (1-y) \log ^{N-1} n+\cdots\right]
$$

with a similar result for $\log y$. Since $\lambda_{N, N}=\lambda / N$, the $\log (1-y)$ in Eq. (3.28) multiplies $\lambda \log ^{N-1} n$, which is comparable to a leading propagator term with one less vertex. 
We will first consider terms containing the factor $\left\langle\left\langle f_{-} \log y\right\rangle\right\rangle$. Looking at such a term arising from $\left\langle(\mathcal{Z}-1)^{p}\right\rangle$, we see that the different factors of $\mathcal{Z}-1$ interact only through the various possible orderings of their $g_{+}$and one $f_{-}$vertices on the main line; thus we can factor these diagrams, and consider only the contribution from a single factor of $\mathcal{Z}-1$ (see Figure 19). This is

$$
\begin{aligned}
\langle\mathcal{Z}-1\rangle_{\left.s \ell, \propto\left\langle f_{-} \log y\right\rangle\right\rangle=} & \sum_{l=0}^{\infty} \sum_{m=1}^{\infty} \frac{1}{(m+l) !}\left(\lambda\left\langle\left\langle f_{-} \log y\right\rangle\right\rangle\right)\left(\lambda\left\langle\left\langle g_{+}\right\rangle\right\rangle \log n\right)^{l}(\lambda\langle\langle\psi\rangle\rangle \log n)^{m} \\
& +\sum_{l=0}^{\infty} \sum_{m=2}^{\infty} \frac{m-1}{(m+l) !}\left(\lambda\left\langle\left\langle f_{-} \log y\right\rangle\right\rangle\right)\left(\lambda\left\langle\left\langle f_{-}\right\rangle\right\rangle \log n\right)\left(\lambda\left\langle\left\langle g_{+}\right\rangle\right\rangle \log n\right)^{l} \\
& \cdot(\lambda\langle\langle\psi\rangle\rangle \log n)^{m-1} .
\end{aligned}
$$

The first term on the right-hand side of Eq. (3.29) corresponds to the case where the factor of $f_{-}$on the main line is averaged with $\log y$, and the second term corresponds to the case where a factor off of the main line is averaged with $\log y$. The sums yield

$$
\langle\mathcal{Z}-1\rangle_{s \ell, \propto\left\langle\left\langle f_{-} \log y\right\rangle\right.}=\lambda^{2}\langle\langle\psi\rangle\rangle \log n\left\langle\left\langle f_{-} \log y\right\rangle\right\rangle \exp (\lambda\langle\langle\psi\rangle\rangle \log n) .
$$

Turning to $\left\langle(\mathcal{Z}-1)^{p}\right\rangle$, we see that there are $p$ choices of which factor of $\mathcal{Z}-1$ contributes the sub-leading term $\propto\left\langle\left\langle f_{-} \log y\right\rangle\right\rangle$. Thus

$$
\begin{aligned}
\langle\log \mathcal{Z}\rangle_{\left.s \ell, \propto\left\langle f_{-} \log y\right\rangle\right\rangle} & =\langle\mathcal{Z}-1\rangle_{\left.s \ell, \propto\left\langle f_{-} \log y\right\rangle\right\rangle} \sum_{p=1}^{\infty} \frac{(-1)^{p-1}}{p} p\left[\langle\mathcal{Z}-1\rangle_{\ell}\right]^{p-1} \\
& =\langle\mathcal{Z}-1\rangle_{s \ell, \propto\left\langle\left\langle f_{-} \log y\right\rangle\right\rangle}\left(\frac{1}{1+\langle\mathcal{Z}-1\rangle_{\ell}}\right) \\
& =\lambda^{2}\langle\langle\psi\rangle\rangle\left\langle\left\langle f_{-} \log y\right\rangle\right\rangle \log n .
\end{aligned}
$$

By contrast, terms $\propto\left\langle\left\langle f_{-} \log (1-y)\right\rangle\right\rangle$ can arise only from a combination of two or more factors of $\mathcal{Z}-1$, since a factor of $f_{-}$is always associated with a weak branch in a single partition function, and hence potentially only with a factor of $\log y$. Consider 
a term arising from a product of $p$ factors of $\mathcal{Z}-1$, each containing $l_{i}$ factors of $g_{+}$ in the main line, followed by one factor of $f_{-}$and then $m_{i}$ factors of $\psi$ off of the main line. Let us further suppose that the factor of $\left\langle\left\langle f_{-} \log (1-y)\right\rangle\right\rangle$ arises from the factor of $f_{-}$appearing in the main line of the $i=1$ factor. Then writing $L=\sum_{i} l_{i}$ and $M=\sum_{i} m_{i}$, we see that any particular sub-leading contribution proportional to $\left\langle\left\langle f_{-} \log (1-y\rangle\right\rangle(\right.$ which we term $\mathcal{D})$ will be given by

$$
\mathcal{D}=\mathcal{N}\left(\lambda\left\langle\left\langle g_{+}\right\rangle\right\rangle \log n\right)^{L}(\lambda\langle\langle\psi\rangle\rangle \log n)^{M}\left(\lambda\left\langle\left\langle f_{-} \log (1-y)\right\rangle\right\rangle\right)\left(\lambda\left\langle\left\langle f_{-}\right\rangle\right\rangle \log n\right)^{p-1},
$$

where the factor $\mathcal{N}$ is determined by the precise ordering of vertices. In fact, it is easy to see that $\mathcal{N}$ will be given by a version of Eq. (3.16) appropriate to $p$ factors of $\mathcal{Z}-1$, with the one addition that there should be a factor of $d / d x_{l_{1}+1}^{(1)}$ inside the integral over the variable $x_{l_{1}+1}^{(1)}$ (and to the right the factor of $\left(x_{l_{1}+1}^{(1)}\right)^{m_{1}} / m_{1}$ !) ). Now the integral of all factors to the right of this new operator will yield some number times $x_{l_{1}+1}^{(1)}$ raised to some power; thus the $d / d x_{l_{1}+1}^{(1)}$ operator can be viewed as acting on only one of the factors of $\mathcal{Z}-1$ at a time. It follows that the sum over permutations of $\mathcal{N}$ can be represented as a sum of averages involving two factors of $\mathcal{Z}-1$ only, with the other factors of $\mathcal{Z}-1$ averaged separately. More precisely,

$$
\langle\log \mathcal{Z}\rangle_{s \ell, \propto\left\langle\left\langle f_{-} \log (1-y)\right\rangle\right\rangle}=\sum_{p=2}^{\infty} \frac{(p-1)}{2}(-1)^{p-1}\left\langle(\mathcal{Z}-1)^{2}\right\rangle_{\left.s \ell, \propto\left\langle f_{-} \log (1-y)\right\rangle\right\rangle}\left[\langle\mathcal{Z}-1\rangle_{\ell}\right]^{p-2}
$$

This naturally represents a considerable simplification of the problem.

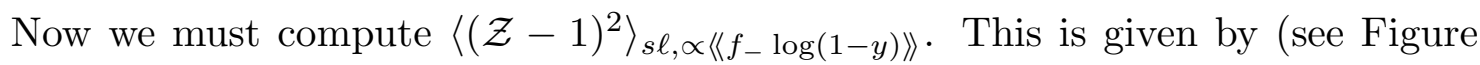
20)

$$
\begin{aligned}
\left\langle(\mathcal{Z}-1)^{2}\right\rangle_{\left.s \ell, \propto\left\langle f_{-} \log (1-y)\right\rangle\right\rangle=2} \sum_{l_{1}=0}^{\infty} \sum_{l_{2}=0}^{\infty} \sum_{m_{1}=0}^{\infty} \sum_{m_{2}=1}^{\infty}\left(\begin{array}{c}
L \\
l_{1}
\end{array}\right) \frac{1}{m_{1} !} \frac{1}{\left(m_{2}-1\right) !} \\
\cdot \frac{(M-1) !}{(M+L) !}\left(\lambda\left\langle\left\langle g_{+}\right\rangle\right\rangle \log n\right)^{L} \\
\cdot(\lambda\langle\langle\psi\rangle\rangle \log n)^{M}\left(\lambda\left\langle\left\langle f_{-} \log (1-y)\right\rangle\right\rangle\right) .
\end{aligned}
$$


where the factor of 2 accounts for the distinguishability of the contributions of the two partition functions. As in the above, $M=m_{1}+m_{2}$, and $L=l_{1}+l_{2}$. The sums may be performed directly, and yield

$$
\begin{aligned}
\left\langle(\mathcal{Z}-1)^{2}\right\rangle_{s \ell, \propto\left\langle\left\langle f_{-} \log (1-y)\right\rangle\right\rangle}= & \lambda\left\langle\left\langle f_{-} \log (1-y)\right\rangle\right\rangle \frac{\langle\langle\psi\rangle\rangle}{\left\langle\left\langle f_{-}\right\rangle\right\rangle} \\
\cdot & {\left[\exp (2 \lambda\langle\langle\psi\rangle\rangle \log n)-\exp \left(2 \lambda\left\langle\left\langle g_{+}\right\rangle\right\rangle \log n\right)\right] . }
\end{aligned}
$$

Since

$$
\begin{aligned}
\sum_{p=2}^{\infty}(-1)^{p-1}(p-1)\left[\langle\mathcal{Z}-1\rangle_{\ell}\right]^{p-2}= & -\left(\frac{1}{1+\langle\mathcal{Z}-1\rangle_{\ell}}\right)^{2} \\
& =-\exp (-2 \lambda\langle\langle\psi\rangle\rangle \log n),
\end{aligned}
$$

our result is

$$
\begin{aligned}
\langle\log \mathcal{Z}\rangle_{\left.s \ell, \propto<\left\langle f_{-} \log (1-y)\right\rangle\right\rangle} & =-\lambda\left\langle\left\langle f_{-} \log (1-y)\right\rangle \frac{\langle\langle\psi\rangle\rangle}{2\left\langle\left\langle f_{-}\right\rangle\right\rangle}\left[1-\exp \left(-2 \lambda\left\langle\left\langle f_{-}\right\rangle\right\rangle \log n\right)\right]\right. \\
& =-\lambda\left\langle\left\langle f_{-} \log (1-y)\right\rangle\right\rangle \frac{\langle\langle\psi\rangle\rangle}{2\left\langle\left\langle f_{-}\right\rangle\right\rangle}\left(1-n^{-2 \lambda\left\langle f_{-}\right\rangle}\right),
\end{aligned}
$$

i.e., a constant plus a decaying power law in $n$.

Terms proportional to $\left\langle\left\langle g_{+} \log (1-y)\right\rangle\right\rangle$ appear at sub-leading order both from one factor of $\mathcal{Z}-1$ and from two factors averaged together. The first case arises from the fact that a $g_{+}$factor has a strong branch associated with it downstream, which can provide a factor of $\log (1-y)$. The combinatorics, and the result, are thus the same as for the term $\propto\left\langle\left\langle f_{-} \log y\right\rangle\right\rangle$, given in Eqs. (3.29-31).

The second case involves the averaging of a $g_{+}$factor arising from one partition function with a factor of $\log (1-y)$ arising from the second partition function, leading to the same combinatorics, and result, as for the term $\propto\left\langle\left\langle f_{-} \log (1-y)\right\rangle\right\rangle$ given in Eqs. (3.33-37). Thus one obtains

$$
\begin{aligned}
\langle\log \mathcal{Z}\rangle_{\left.s \ell, \propto\left\langle g_{+} \log (1-y)\right\rangle\right\rangle}=\lambda\left\langle\left\langle g_{+} \log (1-y)\right\rangle\right\rangle\langle\langle\psi\rangle\rangle \\
\cdot\left\{\lambda \log n-\frac{1}{2\left\langle\left\langle f_{-}\right\rangle\right\rangle}\left[1-\exp \left(-2 \lambda\left\langle\left\langle f_{-}\right\rangle\right\rangle \log n\right)\right]\right\} .
\end{aligned}
$$


We have been discussing sub-leading contributions in which a sub-leading propagator term is taken within the diagram, leading to factors $\log y$ or $\log (1-y)$ being averaged with $f_{-}$or $g_{+}$. In addition, there are sub-leading contributions in which such factors do not appear, but in which $\lambda_{N, N-1}$ appears rather than $\lambda_{N, N} \equiv \lambda / N$. In appendix B we show that

$$
\lambda_{N, N-1}=\frac{1}{2} \frac{\left\langle\left\langle\log ^{2}(1-y)\right\rangle\right\rangle}{\langle\langle\log (1-y)\rangle\rangle^{2}} \equiv \lambda^{\prime},
$$

is independent of $N$. Comparing the expansion of the highest order propagator term, Eq. (3.28), with the form of the full propagator, Eq. (3.4), we note that the inde-

pendence of $N$ of $\lambda_{N, N-1} \equiv \lambda^{\prime}$ allows us to directly translate our previous results to obtain the terms $\propto \lambda^{\prime}$, since the two terms in the $N^{\prime}$ th order propagator $\propto \log ^{N-1} n$ (one including a factor of either $\log y$ or $\log (1-y)$, and the other a factor of $\lambda^{\prime}$ ) are similar in form. In particular, we substitute $\lambda\left\langle\left\langle f_{-} \log y\right\rangle\right\rangle \rightarrow \lambda^{\prime}\left\langle\left\langle f_{-}\right\rangle\right\rangle$in Eq. (3.31), $\lambda\left\langle\left\langle f_{-} \log (1-y)\right\rangle\right\rangle \rightarrow \lambda^{\prime}\left\langle\left\langle f_{-}\right\rangle\right\rangle$in Eq. (3.37), and $\lambda\left\langle\left\langle g_{+} \log (1-y)\right\rangle\right\rangle \rightarrow \lambda^{\prime}\left\langle\left\langle g_{+}\right\rangle\right\rangle$in Eq. (3.38), to obtain

$$
\langle\log \mathcal{Z}\rangle_{s \ell, \propto \lambda^{\prime}}=\lambda^{\prime}\langle\langle\psi\rangle\rangle^{2}\left[\lambda \log n-\frac{1}{2\left\langle\left\langle f_{-}\right\rangle\right\rangle}\left(1-n^{-2 \lambda\left\langle\left\langle f_{-}\right\rangle\right.}\right)\right],
$$

which is again a combination of a logarithmic divergence, a constant, and a decaying power law.

\section{Summation of sub-leading terms: Non-universal}

At sub-leading order, there are also non-universal terms. These arise from taking one factor of $a_{0} \equiv \lambda_{1,0}$ instead of $\lambda \log n$ in the first propagator sum in a diagram; taking non-universal terms in any propagator sum upstream of this will lead at most to sub-sub-leading terms. Reviewing the derivation of Eq. (3.6), we see that

$$
\langle\mathcal{Z}-1\rangle_{s \ell, \propto a_{0}}=a_{0}\langle\langle\psi\rangle\rangle \sum_{N=0}^{\infty} \frac{1}{N !}(\lambda\langle\langle\psi\rangle\rangle \log n)^{N}=a_{0}\langle\langle\psi\rangle\rangle \exp (\lambda\langle\langle\psi\rangle\rangle \log n) .
$$

Note that this summation starts at $N=0$. It follows immediately, using the methods developed above to prove factorization of averages of products of $\mathcal{Z}-1$, that 
$\langle\log \mathcal{Z}\rangle_{s \ell, \propto a_{0}}=\langle\mathcal{Z}-1\rangle_{s \ell, \propto a_{0}} \sum_{p=1}^{\infty}(-1)^{p-1}\left[\langle\mathcal{Z}-1\rangle_{\ell}\right]^{p-1}=a_{0}\langle\langle\psi\rangle\rangle$

\section{Summary of leading and sub-leading orders}

To summarize, we have the following result for $\langle\log \mathcal{Z}\rangle$, combining the leading order, sub-leading universal, and sub-leading non-universal computations (Eqs. (3.19), $(3.26),(3.31),(3.37-8),(3.40)$, and $(3.42))$ :

$$
\begin{aligned}
\langle\log \mathcal{Z}\rangle=\lambda\langle\langle\psi\rangle\rangle \log n\left[1+\lambda\left(\left\langle\left\langle g_{+} \log (1-y)\right\rangle\right\rangle+\left\langle\left\langle f_{-} \log y\right\rangle\right\rangle\right)+\lambda^{\prime}\langle\langle\psi\rangle\rangle\right] \\
-\frac{1}{2\left\langle\left\langle f_{-}\right\rangle\right\rangle}\left[1-n^{\left.-2 \lambda\left\langle f_{-}\right\rangle\right]}\right. \\
\quad \cdot\left(\frac{1}{2}\left\langle\left\langle\psi^{2}\right\rangle\right\rangle+\lambda\langle\langle\psi\rangle\rangle\langle\langle\psi \log (1-y)\rangle\rangle+\lambda^{\prime}\langle\langle\psi\rangle\rangle^{2}\right) \\
+a_{0}\langle\langle\psi\rangle\rangle+O\left(\delta^{3} \log n\right),
\end{aligned}
$$

where the $O\left(\delta^{3} \log n\right)$ terms are sub-sub-leading (see Table I).

Examining in detail the re-summations performed above, we see that all of the terms contributing to the coefficient of $\log n$ in Eq. (3.43) arise from separate averages of single factors of $\mathcal{Z}-1$; averages together of two factors contribute to the second term on the right-hand side of Eq. (3.43). Anticipating that this is true to all orders, we see that ${ }^{2}$

$$
\lim _{n \rightarrow \infty} \frac{\langle\log \mathcal{Z}\rangle}{\log n}=\lim _{n \rightarrow \infty} \frac{\log \langle\mathcal{Z}\rangle}{\log n} .
$$

Thus, if we are concerned only with the term proportional to $\log n$, which should dominate as $n \rightarrow \infty$, this can be obtained from $\log \langle\mathcal{Z}\rangle$. This quantity is computed by much less elaborate means in appendix D.

2 Using the terminology of statistical mechanics, averages that can be reduced to individual averages $\langle\mathcal{Z}-1\rangle$ may be termed disconnected, while those involving averages together of more than one factor of $\mathcal{Z}-1$ may be termed connected. Thus Eq. (3.44) corresponds to a sum of disconnected averages only. 
There are two methods of checking Eq. (3.43), which resums all terms of the form $\delta^{N} \log ^{N} n$ or $\delta^{N} \log ^{N-1} n$, with $N \geq 1$. The power laws in $n$ may be converted into an expansion in $\log n$, and then compared directly with the $\delta$ expansion, as developed to second order in section 2 above, and to fourth order in appendix B. The reader may easily satisfy himself that these two series agree, provided that comparison is confined to terms proportional to $\delta^{N} \log ^{N} n$ or $\delta^{N} \log ^{N-1} n$, in this case with $1 \leq N \leq 4$.

A second method of checking this result is to recall that the dependence of the partition function $\mathcal{Z}$ upon $\sigma$ is quite trivial: $\mathcal{Z} \propto n^{\sigma}$ (see Eqs. (1.7) and (1.10)). However, in our perturbative method this $\sigma$-dependence is distributed throughout the branched tree, with a factor of $y^{\sigma}$ or $(1-y)^{\sigma}$ corresponding to this $\sigma$ dependence appearing at each vertex. This considerably obscures the $\sigma$ dependence, but may also be used to check the final result for $\langle\log \mathcal{Z}\rangle$, Eq. (3.43). Let us write again Eq. (1.10),

$$
\langle\log \mathcal{Z}(q, \sigma ; n)\rangle=\sigma \log n+F(q, n)
$$

where $F$ has no dependence upon $\sigma$. This implies that

$$
\frac{d\langle\log \mathcal{Z}\rangle}{d \sigma}=\log n
$$

and

$$
\frac{d^{r}\langle\log \mathcal{Z}\rangle}{d \sigma^{r}}=0, \quad r>1
$$

These relations provide powerful constraints on our result. To check these identities against our result for $\langle\log \mathcal{Z}\rangle$, Eq. (3.43), it is necessary to use (see Eqs. (2.3) and (2.11))

$$
\frac{d g_{+}}{d \sigma}=-\left(g_{+}+1\right) \log (1-y)
$$

and

$$
\frac{d f_{-}}{d \sigma}=-f_{-} \log (y) .
$$


Note that due to Eq. (3.48), taking the derivative with respect to $\sigma$ effectively reduces the order in $\delta$ of terms involving $g_{+}$by one. Thus we can only compute derivatives with respect to $\sigma$ of Eq. (3.43) to $O(\delta)$.

Let us rewrite Eq. (3.43) as

$$
\langle\log \mathcal{Z}\rangle=A(q, \sigma) \log n+B(q, \sigma)\left(1-n^{-2 \lambda\left\langle f_{-}\right\rangle}\right)+C(q, \sigma)+\cdots
$$

where the coefficients $A(q, \sigma), B(q, \sigma)$, and $C(q, \sigma)$ can be read from Eq. (3.43), and the coefficient $C=a_{o}\langle\langle\psi\rangle\rangle$ is the only non-universal one. Taking the derivatives with respect to $\sigma$, we see after some computation that indeed

$$
\frac{d A(q, \sigma)}{d \sigma}=1+O\left(\delta^{2}\right)
$$

and

$$
\frac{d B(q, \sigma)}{d \sigma}=O\left(\delta^{2}\right)
$$

Thus Eqs. (3.46-7) are satisfied provided that the non-universal constant $a_{0}=0$. It seems that we have succeeded in fixing this quantity. However, we remind the reader that many cut-off procedures will effectively break the invariance expressed by Eqs. (3.45-47) by introducing a dependence on the number of particles in an elementary branch $n_{e}$, so the result $a_{0}=0$ should not be taken too seriously.

\section{Interpretation of results}

In section 3, we re-summed the leading and sub-leading divergent series to all orders in perturbation theory. We found a remarkable result. The resummed series consists of three types of terms. In the first place, there are terms diverging logarithmically with $n$. These terms are all universal in form. In the second place, there are constant terms-at sub-leading order these include non-universal constants. Finally, there are power law corrections to $\langle\log \mathcal{Z}\rangle$, which involve universal coefficients multiplying $n^{-\Delta}$, with 


$$
\Delta=2 \lambda\left\langle\left\langle f_{-}\right\rangle\right\rangle
$$

a positive quantity. Thus as $n \rightarrow \infty$ we expect only the terms $\propto \log n$ to be relevant in determining the quenched dimensions $\sigma_{\mathcal{Q}}(q)$. Recalling the coefficient of this term from Eq. (3.43), we conclude that

$$
\begin{aligned}
\left\langle\left\langle\psi\left(q, \sigma_{\mathcal{Q}}(q)\right)\right\rangle\right\rangle\left\{1+\lambda\left[\left\langle\left\langle g_{+}\left(q, \sigma_{\mathcal{Q}}(q)\right) \log (1-y)\right\rangle\right\rangle\right.\right. & \left.+\left\langle\left\langle f_{-}\left(q, \sigma_{\mathcal{Q}}(q)\right) \log y\right\rangle\right\rangle\right] \\
& \left.+\lambda^{\prime}\left\langle\left\langle\psi\left(q, \sigma_{\mathcal{Q}}(q)\right)\right\rangle\right\rangle+O\left(\delta^{2}\right)\right\}=0
\end{aligned}
$$

which is equivalent to $\left\langle\left\langle\psi\left(q, \sigma_{\mathcal{Q}}(q)\right)\right\rangle\right\rangle=0$. (Setting the other factor equal to zero does not lead to an acceptable solution. $)^{3}$ But this is precisely the same as the criterion that determines the annealed multifractal dimensions $\sigma_{\mathcal{A}}(q)$ ! This is unsurprising, as the $\log n$ term in Eq. (3.43) resulted only from products of single averages of $\langle\mathcal{Z}-1\rangle$, as discussed in section 3 or in appendix D. This implies that the true $n \rightarrow \infty$ quenched multifractal dimensions are identical to the annealed dimensions, negative values of $f$ and all.

However, this conclusion is based upon an overly naive interpretation of Eq. (3.43), overlooking crossover effects, which we now discuss. Consider again the power law term $\propto n^{-\Delta}$, with $\Delta$ given by Eq. (4.1). We can understand the role played by this term by considering model $\mathrm{Z}$, in which the unstable manifold for branch competition in the $x-y$ plane is simply a straight line emanating from the unstable fixed point at $(1 / 2,1 / 2)$, which turns vertical upon reaching the $x=0$ or $x=1$ lines (see Figure 3 ). As a function of $\eta$, we can write ${ }^{(11)}$

\footnotetext{
3 Numerical evaluation of the second factor on the right-hand side of Eq. (4.2) for, e.g., model Z (see section 1 or Eqs. (4.3-4)) quickly leads one to the conclusion that setting this factor equal to zero does not lead to a physical solution for $\sigma_{\mathcal{Q}}(q)$, or, indeed, to any solution for some values of $q$.
} 


$$
x= \begin{cases}\frac{1}{2}\left(1-\eta^{\nu}\right), & \eta<1 \\ 0, & \eta \geq 1\end{cases}
$$

and

$$
y= \begin{cases}\frac{1}{2}\left\{1-\left[\eta^{\nu} /(1+\nu)\right]\right\}, & \eta<1 \\ \bar{\eta} / \eta, & \eta \geq 1\end{cases}
$$

with $\bar{\eta}=[\nu / 2(1+\nu)]$.

Recalling the definition of $\left\langle\left\langle f_{-}\right\rangle\right\rangle$,

$$
\left\langle\left\langle f_{-}\right\rangle\right\rangle=\int_{0}^{\infty} d \eta \eta^{\nu-1} \frac{x^{q}(\eta)}{y^{\sigma}(\eta)}
$$

we see that in general, $\left\langle\left\langle f_{-}\right\rangle\right\rangle$is a function of both $q$ and $\sigma$. This presents us with a paradox, because any exponent $\Delta$ of a power law correction to $\langle\log \mathcal{Z}\rangle$ must be independent of $\sigma$, due to the invariances expressed by Eqs. (3.45-47). We resolve this paradox by realizing that Eq. (4.1) is merely the lowest order in $\delta$ expression for the exponent $\Delta$; we should write (4.1) more precisely as

$$
\Delta(q)=2 \lambda\left\langle\left\langle f_{-}\right\rangle\right\rangle+O\left(\delta^{2}\right)
$$

where $\Delta$ is now the exact exponent of the leading power law correction to $\langle\log \mathcal{Z}\rangle$. The higher order corrections on the right-hand side of Eq. (4.6) will cancel the dependence upon $\sigma$ arising from $\left\langle\left\langle f_{-}\right\rangle\right\rangle$.

Let us first compute compute $\left\langle\left\langle f_{-}\right\rangle\right\rangle$in model $\mathrm{Z}$ for $\sigma=0$,

$$
\left\langle\left\langle f_{-}(q, \sigma=0)\right\rangle\right\rangle=\frac{1}{\nu(q+1)}\left(\frac{1}{2}\right)^{q},
$$

obtaining

$$
\Delta(q)=\frac{2 \lambda}{\nu(q+1)}\left(\frac{1}{2}\right)^{q}+O\left(\delta^{2}\right),
$$

which goes to zero exponentially in $q$ for large $q$.

Now consider $\langle\log \mathcal{Z}\rangle$ from Eq. (3.43). For convenience, we set the non-universal constant $a_{0}=0$. If $\lim _{q \rightarrow \infty} \Delta(q)=0$, we see that (returning to the case of general $\sigma$ ), 


$$
\begin{aligned}
\lim _{q \rightarrow \infty} \lim _{n \rightarrow \infty} \frac{\langle\log \mathcal{Z}\rangle_{\ell+s \ell}}{\log n}=\lim _{q \rightarrow \infty} \lambda\langle\langle\psi\rangle\rangle\left[1+\lambda\left(\left\langle\left\langle g_{+} \log (1-y)\right\rangle\right\rangle\right.\right. & \left.+\left\langle\left\langle f_{-} \log y\right\rangle\right\rangle\right) \\
& \left.+\lambda^{\prime}\langle\langle\psi\rangle\rangle\right]
\end{aligned}
$$

while

$$
\begin{aligned}
\lim _{n \rightarrow \infty} \lim _{q \rightarrow \infty} \frac{\langle\log \mathcal{Z}\rangle_{\ell+s \ell}}{\log n}= & \lim _{q \rightarrow \infty}\left\{\lambda\langle\langle\psi\rangle\rangle\left[1+\lambda\left(\left\langle\left\langle g_{+} \log (1-y)\right\rangle\right\rangle+\left\langle\left\langle f_{-} \log y\right\rangle\right\rangle\right)+\lambda^{\prime}\langle\langle\psi\rangle\rangle\right]\right. \\
& \left.\left.-\lambda\left[\frac{1}{2}\left\langle\left\langle\psi^{2}\right\rangle\right\rangle+\lambda\langle\langle\psi\rangle\rangle\langle\psi \psi \log (1-y)\rangle\right\rangle+\lambda^{\prime}\langle\langle\psi\rangle\rangle^{2}\right]\right\} \\
= & \lim _{q \rightarrow \infty}\left[\lambda\langle\langle\psi\rangle\rangle+\lambda^{2}\langle\langle\psi\rangle\rangle\left\langle\left\langle f_{-} \log \left(\frac{y}{1-y}\right)\right\rangle\right\rangle-\frac{\lambda}{2}\left\langle\left\langle\psi^{2}\right\rangle\right\rangle\right]
\end{aligned}
$$

because

$$
\lim _{n \rightarrow \infty} \lim _{q \rightarrow \infty}\left(1-n^{-\Delta(q)}\right)=2 \lambda\left\langle\left\langle f_{-}\right\rangle\right\rangle \log n+O\left(\delta^{2}\right) .
$$

Thus, for large values of $q$, the apparent quenched dimensions, which we term $\sigma_{\mathcal{P}}(q)$, will be determined by

$$
\left.\lambda\langle\langle\psi\rangle\rangle+\lambda^{2}\langle\langle\psi\rangle\rangle\left\langle f_{-} \log \left(\frac{y}{1-y}\right)\right\rangle\right\rangle-\frac{\lambda}{2}\left\langle\left\langle\psi^{2}\right\rangle\right\rangle+O\left(\delta^{3}\right)=0 .
$$

These apparent dimensions are the ones that will be seen, e.g., in numerical studies. Note that this is simply the naive (non-resummed) result for the dimensions $\sigma_{\mathcal{Q}}(q)$ which cam be obtained from $\langle\log \mathcal{Z}\rangle=\langle\log \mathcal{Z}\rangle_{1}+\langle\log \mathcal{Z}\rangle_{2}+O\left(\delta^{3}\right)$ (see Eqs. (2.20) and $(2.34))$.

Of course, for sufficiently large $n$, we will always see a crossover back to the true quenched dimensions. The crossover value of $n$ for which this occurs, $n_{c}(q)$, will be determined by

$$
\Delta(q) \log n_{c}(q)=\left[2 \lambda\left\langle\left\langle f_{-}\right\rangle\right\rangle+O\left(\delta^{2}\right)\right] \log n_{c}(q)=1,
$$

or, from Eq. (4.7), 


$$
n_{c}(q)=\exp \left[\frac{\nu(q+1) 2^{q}}{2 \lambda}+O\left(\delta^{0}\right)\right]
$$

(Note that the first term in the exponential in Eq. (4.14) is formally $O\left(\delta^{-1}\right)$.) Thus, in practice, the true quenched dimensions will not be observable, even for moderate values of $q$, except for monstrously large systems. Although we have taken an explicit form for $\Delta$ appropriate for model $\mathrm{Z}$, we expect the same qualitative results to hold for any branched growth model, and in particular, for DLA.

Suppose that we had not set $\sigma=0$ at the outset of this calculation. It is simple to compute $\left\langle\left\langle f_{-}(q, \sigma)\right\rangle\right\rangle$ for model $\mathrm{Z}$,

$$
\left\langle\left\langle f_{-}(q, \sigma)\right\rangle\right\rangle=\frac{1}{\nu}\left(\frac{1}{2}\right)^{q-\sigma}\left(1+\frac{1}{\nu}\right)^{\sigma} \int_{0}^{1} d u u^{q}\left(1+\frac{u}{\nu}\right)^{-\sigma}
$$

and

$$
\lim _{q \rightarrow \infty}\left\langle\left\langle f_{-}(q, \sigma)\right\rangle\right\rangle=\frac{1}{\nu(q+1)}\left(\frac{1}{2}\right)^{q-\sigma}
$$

which is a simple modification of our previous result, Eq. (4.7). For fixed $\sigma$, Eq. (4.16) leads to the same super-exponential dependence of $n_{c}$ on $q$ displayed in Eq. (4.14). We expect the annealed $\sigma_{\mathcal{A}}(q)$ to approach a constant as $q \rightarrow \infty$, while $\sigma_{\mathcal{P}}(q) \rightarrow \beta q, \beta \approx$ $1-\nu<1$ for $q \rightarrow \infty$. $^{(11)}$ It is not, however, legitimate to introduce these functions $\sigma(q)$ into Eq. (4.16), because once $\sigma$ becomes large, we must properly include the effects of the next order term in $\delta$ in Eq. (4.14), which will offset this spurious $\sigma$-dependence.

\section{Numerical results}

In addition to the analytical computation of $\langle\log \mathcal{Z}\rangle$, to which the bulk of this work is devoted, we have also computed this quantity, for the random branched growth model, by a Monte Carlo method. For these purposes, it is convenient to use model Z, which can be defined (see Figure 3) by writing $x$ and $y$ as functions of $\eta$ as in Eqs. (4.3-4). The curve in the $x-y$ plane parameterized by these functions (and their complements $1-x, 1-y$ ) is precisely the unstable manifold shown in Figure 3.

We must also fix the function $\rho(\epsilon)$. We expect our results to be sensitive only to the $\epsilon \ll 1$ form of $\rho(\epsilon) \propto \rho_{0} \epsilon^{\nu-1}$. It is thus convenient to choose 


$$
\rho(\epsilon)=\frac{\epsilon^{\nu-1} e^{-\epsilon}}{\Gamma(\nu)},
$$

as a suitably normalized form for $\rho(\epsilon)$.

Our procedure is now quite simple. Starting at the first branch point, at the root of the tree, we fix $n$, the total number of particles in the tree. We then choose a value of $\epsilon$ with the distribution given by Eq. (4.17), and compute the values of $x$ and $y$ at that branch point from Eqs. (4.3-4). We then repeat the process on each of the two sub-branches, and so forth. If the total probability on a branch is zero (as will be the case for values of $\eta \geq 1$ on the weaker branch in model $\mathrm{Z}$ ), then it will not contribute to $\sum_{i} p_{i}^{q}$, and it can be disregarded. If the total mass of a branch is less than one, then we stop the subdivision process. We then form the sum either of the probability moments of these ends, which correspond to the elementary sub-branches (indexed by i),

$$
Z(q)=\sum_{i} p_{i}^{q}
$$

or else the sum weighted by a simple function of the mass of each elementary subbranch

$$
Z^{\prime}(q)=\sum_{i} \frac{p_{i}^{q}}{n_{i}^{q-1}}
$$

We have used the factor $n_{i}^{1-q}$ on the right-hand side of Eq. (4.19) because $\sigma_{\mathcal{A}}(q)=$ $\sigma_{\mathcal{Q}}(q)=q-1$ for a non-fractal cluster, and the elementary sub-branches are presumably not fractals. Numerical results for the scaling behavior of these two versions of the partition function were, for practical purposes, identical. We can now determine the "apparent" dimensions $\sigma_{\mathcal{P}}(q)$ by studying the scaling of $\langle\log Z(q)\rangle$ with $\log n$, where $\langle\cdots\rangle$ indicates the average over the statistically independent cluster realizations.

Although we performed simulations for a variety of values of $0<\nu<1$, we shall display our results only for $\nu=0.6$ (results for other values of $\nu$ are qualitatively similar). We used values of $n$ varying from $n=10$ to $n=2560$, varying by powers of 
two, and we averaged over $10^{4}$ realizations at each size. Figure 21 displays $\langle\log Z\rangle$ vs. $\log n$ for two values of $q$. The linear scaling is quite evident in these plots. Extracting the slope of the linear part, we find $\sigma_{\mathcal{P}}(q)$ as displayed in Figure 22, with its corresponding $f(\alpha)$ (defined as the Legendre transform of $\sigma_{\mathcal{P}}(q)$, not of $\tau(q)$-see section 1 above for a discussion of the relation between these two quantities). Note that the $f(\alpha)$ corresponding to the numerical quenched dimensions does not have any negative values of $f$ appearing.

We can implicitly solve Eqs. (4.2) or (4.12) above in order to find $\sigma_{\mathcal{A}}(q)$ or the apparent quenched dimensions $\sigma_{\mathcal{P}}(q)$. These are displayed with the numerical results in Figure 23. For comparison, we also show the one-propagator result from Eq. (2.37). It is clear that the annealed dimensions agree with the numerical results only for $q<2$, while the apparent quenched dimensions from Eq. (4.12) agree over the larger range $q<5$. From Eq. (4.14) for $n_{c}(q)$, we already expect that for $q=3$ the crossover from apparent quenched to annealed dimensions (for model $\mathrm{Z}$ with $\nu=0.6$ ) in the numerical results will occur only for $n \gg 10^{5}$. Since these dimensions are essentially identical for $q<3$, it is not feasible to see this crossover in the numerical results.

It is possible to understand the fact that for $q$ not too large, the $\sigma_{\mathcal{P}}(q) \approx \sigma_{\mathcal{A}}(q)$, by recasting the expansion in the formal parameter $\delta$ as an expansion in $q-1$. In this way, one can show that $\sigma_{\mathcal{P}}(q)=\sigma_{\mathcal{A}}(q)+O\left((q-1)^{2}\right)$. This is discussed in detail in appendix E.

\section{Conclusions}

We now summarize the main results of this work, which concern the partition function $\mathcal{Z}(q, \sigma ; n)$ defined in $\operatorname{Eq}(2.2)$.

1. The multifractal dimensions for the branched growth model may be defined either by annealed averaging, for which $\lim _{n \rightarrow \infty}\left\langle\mathcal{Z}\left(q, \sigma_{\mathcal{A}}(q)\right)\right\rangle=0$, or by quenched averaging, $\lim _{n \rightarrow \infty}\left\langle\log \mathcal{Z}\left(q, \sigma_{\mathcal{Q}}(q)\right)\right\rangle=0$.

2. It is possible to compute $\langle\log \mathcal{Z}\rangle$ perturbatively by expanding in a formal parameter $\delta$, which counts branching vertices in a diagrammatic approach. The result is a formal expression 


$$
\langle\log \mathcal{Z}\rangle=\sum_{N=1}^{\infty} \delta^{N}\langle\log \mathcal{Z}\rangle_{N}=\sum_{N=1}^{\infty} \sum_{M=0}^{N} \beta_{N, M} \log ^{M} n
$$

where the parameter $\delta=1$ in the physical case and the coefficients $\left\{\beta_{N, M}\right\}$ can be determined from the unstable manifold for branch competition in the underlying model.

3. It is possible to resum the leading $M=N$ and sub-leading $M=N-1$ terms at all orders $N$ in Eq. (5.1). The result is an expression for $\langle\log \mathcal{Z}\rangle$ which combines a logarithmic term in $n$ with a constant and a decaying power law:

$$
\langle\log \mathcal{Z}(q, \sigma, n)\rangle=\langle\langle\psi(q, \sigma)\rangle\rangle \Gamma_{0}(q, \sigma) \log n+\Gamma_{1}(q, \sigma)\left(1-n^{-\Delta}\right)+\cdots
$$

with $\Delta(q)>0$.

4. From Eq. (5.2), we see that in the limit $n \rightarrow \infty, \sigma_{\mathcal{Q}}(q)$ is fixed by requiring $\langle\langle\psi\rangle\rangle=0$, which is also the criterion that fixes $\sigma_{\mathcal{A}}(q)$. Thus, in the limit of large $n, \sigma_{\mathcal{Q}}(q)=\sigma_{\mathcal{A}}(q)$. This limit is attained only for $n \gg n_{c}=\exp (a \exp b q)$. For $n \ll n_{c}$, the apparent multifractal dimensions $\sigma_{\mathcal{P}}(q) \neq \sigma_{\mathcal{A}}(q)$. Furthermore, there is numerical evidence that, unlike the annealed dimensions, these apparent dimensions do not exhibit negative values of $f(\alpha)$.

5. For appreciable values of $q-1, n_{c}$ is enormous, so that the true quenched (i.e., annealed) dimensions are virtually unobservable. For $q-1 \sim 1$, the true quenched dimensions will be observed; but in this case $\sigma_{\mathcal{P}}(q) \rightarrow \sigma_{\mathcal{A}}(q)$ anyway.

The multifractality of these models is of a quite interesting kind. The average of the partition function displays true scaling over the entire range of $q$. The average of its logarithm, on the other hand, contains regions above and below $n_{c}$ with different scaling properties. As a function of $\mathrm{q}$, there is a weak function $q_{c}(n)$ such that for $q<q_{c}$ and $q>q_{c}$ essentially different scaling properties are being explored. Since the essence of multifractality is the smooth variation of scaling properties with $q$, this represents, in a subtle sense, a failure of multifractality. 
It is therefore most accurate to say that these branched growth models are multifractal only as an ensemble; typical members of the ensemble exhibit this weak deviation from multifractality. Since the branched growth model seems to be a quite adequate theory, both qualitatively and quantitatively, for diffusion-limited aggregation, we expect these quite novel properties to hold also for DLA.

\section{Acknowledgements}

We are grateful to A. Coniglio and M. Leibig for stimulating discussions. T.C. Halsey would like to thank the Dipartimento di Fisica, Università di Napoli, and the Institute for Theoretical Physics, Santa Barbara for their hospitality. He is also grateful for the support of the National Science Foundation, through a Presidential Young Investigator Grant, DMR-9057156, and through grant PHY89-04035, as well as for the support of an Alfred P. Sloan junior fellowship. K. Honda would like to thank the Ministry of Education, Science, and Culture of Japan for a travelling grant. B. Duplantier is grateful for the support of the CNRS through the CNRS-NSF FranceU.S. Binational Program. 


\section{Appendix A: "Propagator" sums}

It was claimed above in section 2 that

$$
\left\langle\sum_{j \geq 1} \frac{\rho_{0} \log ^{N-1} n_{j}}{n_{j}^{\nu}}\right\rangle=\sum_{M=0}^{N} \lambda_{N, M} \log ^{M} n
$$

where the sum over $j$ is down a "main line" with a total of $n$ particles. First we repeat the computation of $\lambda_{1,1}$. From Eq. (A.1)

$$
\left\langle\sum_{j \geq 1} \frac{\rho_{0}}{n_{j}^{\nu}}\right\rangle=\lambda_{1,1} \log n+\lambda_{1,0}
$$

Separating out the first term in the sum, we have

$$
\lambda_{1,1} \log n+\lambda_{1,0}=\frac{\rho_{0}}{n^{\nu}}+\left\langle\lambda_{1,1} \log [(1-y) n]+\lambda_{1,0}\right\rangle
$$

which simplifies to

$$
\frac{\rho_{0}}{n^{\nu}}+\left\langle\lambda_{1,1} \log (1-y)\right\rangle=0
$$

or (recalling Eqs. (2.7), (2.8))

$$
\lambda_{1,1} \equiv \lambda=-\frac{1}{\langle\langle\log (1-y)\rangle\rangle},
$$

The factor of $\rho_{0}$ has cancelled against that coming from the integral, so that $\lambda_{1,1}$ is independent of $\rho_{0}$, as are all of the $\left\{\lambda_{N, M}\right\}$. The constant $\lambda_{1,0}$ (elsewhere called $\left.a_{0}\right)$ is not determined by this argument; in fact we expect it to depend on the specific cut-off procedure in the theory, and thus be non-universal.

To compute the remaining $\left\{\lambda_{N, M}\right\}$, we simply generalize the above argument. 


$$
\begin{aligned}
\left\langle\sum_{j \geq 1} \frac{\rho_{0} \log ^{N-1} n_{j}}{n_{j}^{\nu}}\right\rangle & =\sum_{M=0}^{N} \lambda_{N, M} \log ^{M} n \\
& =\frac{\rho_{0} \log ^{N-1} n}{n^{\nu}}+\left\langle\sum_{M=0}^{N} \lambda_{N, M} \log ^{M}[(1-y) n]\right\rangle \\
& =\frac{\rho_{0} \log ^{N-1} n}{n^{\nu}}+\sum_{M=0}^{N} \lambda_{N, M} \sum_{L=0}^{M}\left(\begin{array}{c}
M \\
L
\end{array}\right) \log ^{L} n\left\langle\log ^{M-L}(1-y)\right\rangle
\end{aligned}
$$

leading immediately to

$$
\log ^{N-1} n+\sum_{M=1}^{N} \lambda_{N, M} \sum_{L=0}^{M-1}\left(\begin{array}{c}
M \\
L
\end{array}\right) \log ^{L} n\left\langle\left\langle\log ^{M-L}(1-y)\right\rangle\right\rangle=0
$$

The left hand side of Eq. (A.7) is a sum of terms of the form $a_{K} \log ^{K} n$, with $0 \leq$ $K \leq N-1$. Setting each of the coefficients $a_{K}$ equal to zero yields $N$ simultaneous linear equations for the $N$ quantities $\left\{\lambda_{N, M}, M \geq 1\right\}$. The solution of these equations then gives $\left\{\lambda_{N, M}, M \geq 1\right\}$. The non-universal coefficients $\left\{\lambda_{N, 0}\right\}$ do not appear in Eq. (A.7), and are left un-determined by this procedure.

As an example, consider $\lambda_{N, N}$. These may be obtained from the coefficient of the $\log ^{N-1} n$ term in Eq. (A.7),

$$
\lambda_{N, N}=-\frac{1}{N\langle\langle\log (1-y)\rangle\rangle}=\frac{\lambda_{1,1}}{N} \equiv \frac{\lambda}{N} .
$$

It is also simple to determine the $\left\{\lambda_{N, N-1}\right\}$. The coefficient of the $\log ^{N-2} n$ term in Eq. (A.7) is

$$
\lambda_{N, N-1}\left(\begin{array}{c}
N-1 \\
N-2
\end{array}\right)\langle\langle\log (1-y)\rangle\rangle+\lambda_{N, N}\left(\begin{array}{c}
N \\
N-2
\end{array}\right)\left\langle\left\langle\log ^{2}(1-y)\right\rangle\right\rangle=0
$$

giving

$$
\lambda_{N, N-1}=-\lambda_{N, N} \frac{N}{2} \frac{\left\langle\left\langle\log ^{2}(1-y)\right\rangle\right\rangle}{\langle\langle\log (1-y)\rangle\rangle}=\frac{1}{2} \frac{\left\langle\left\langle\log ^{2}(1-y)\right\rangle\right\rangle}{\langle\langle\log (1-y)\rangle\rangle^{2}} \equiv \lambda^{\prime} .
$$


The next coefficient, that for the $\log ^{N-3} n$ term from Eq. (A.7), gives

$$
\lambda_{N, N-2}=(N-1)\left\{\frac{1}{6} \frac{\left\langle\left\langle\log ^{3}(1-y)\right\rangle\right\rangle}{\langle\langle\log (1-y)\rangle\rangle^{2}}-\frac{1}{4} \frac{\left\langle\left\langle\log ^{2}(1-y)\right\rangle\right\rangle^{2}}{\langle\langle\log (1-y)\rangle\rangle^{3}}\right\} .
$$

Clearly, one may iterate to generate any of the $\lambda_{N, M}$ that one needs.

\section{Appendix B: Series in $\delta$ to fourth order}

In section 2 above, we demonstrated the rules for computing $\langle\log \mathcal{Z}\rangle$ order by order in $\delta$, and computed the terms of $O(\delta)$ and $O\left(\delta^{2}\right)$. The diagrams corresponding to these terms are displayed in Figure 24, the results are

$$
\langle\log \mathcal{Z}\rangle_{1}=\left(\lambda \log n+a_{0}\right)\langle\langle\psi\rangle\rangle
$$

and

$$
\langle\log \mathcal{Z}\rangle_{2}=\left(\lambda \log n+a_{0}\right)\left[\lambda\langle\langle\psi\rangle\rangle\left\langle\left\langle f_{-} \log \left(\frac{y}{1-y}\right)\right\rangle\right\rangle-\frac{1}{2}\left\langle\left\langle\psi^{2}\right\rangle\right\rangle\right]
$$

Recall that in our more formal notation, $\lambda \equiv \lambda_{1,1}$ and the non-universal constant $a_{0} \equiv \lambda_{1,0}$. In the expressions below, we will find it convenient to mix these two notations, as well as to use $\lambda^{\prime} \equiv \lambda_{2,1}$.

At third order in $\delta$, the diagrams appearing are shown in Figure 25. The attentive reader will recall that each diagram will in general involve a number of terms, due to the different ways in which propagator terms can be averaged with vertices $f_{-}$or $g_{+}$. A lengthy computation yields the following result for $\langle\log \mathcal{Z}\rangle_{3}$ : 


$$
\begin{aligned}
\langle\log \mathcal{Z}\rangle_{3}=( & \left.\lambda_{2,2} \log ^{2} n+\lambda_{2,1} \log n+\lambda_{2,0}\right) \\
\cdot & {\left[2 \lambda\left\langle\left\langle f_{-}\right\rangle\right\rangle\left\langle\langle\psi\rangle\left(\lambda\langle\langle\psi \log (1-y)\rangle\rangle+\lambda^{\prime}\langle\langle\psi\rangle\rangle\right)+\lambda\left\langle\left\langle f_{-}\right\rangle\right\rangle\left\langle\left\langle\psi^{2}\right\rangle\right\rangle\right]\right.} \\
+ & \left(\lambda_{1,1} \log n+\lambda_{1,0}\right) \\
& \cdot\left\{\lambda ^ { 2 } \langle \langle \psi \rangle \rangle \left(\frac{1}{2}\langle\langle\psi\rangle\rangle\left\langle\left\langle f_{-} \log ^{2}\left(\frac{y}{1-y}\right)\right\rangle\right\rangle+\left\langle\left\langle f_{-} \log \left(\frac{y}{1-y}\right)\right\rangle\right\rangle^{2}\right.\right. \\
& \left.\left.+\left\langle\left\langle f_{-} \log [y(1-y)]\right\rangle\right\rangle\langle\psi \log (1-y)\rangle\right\rangle\right) \\
+ & \lambda \lambda^{\prime}\langle\langle\psi\rangle\rangle^{2}\left\langle\left\langle f_{-} \log [y(1-y)]\right\rangle\right\rangle+\lambda\left\langle\left\langle\psi^{2}\right\rangle\right\rangle\left\langle\left\langle f_{-} \log (1-y)\right\rangle\right\rangle \\
& \left.-\lambda\langle\langle\psi\rangle\rangle\left\langle\left\langle f_{-} \psi \log \left(\frac{y}{1-y}\right)\right\rangle\right\rangle+\frac{1}{3}\left\langle\left\langle\psi^{3}\right\rangle\right\rangle\right\} \\
+ & \left(\lambda_{1,1} \log n+\lambda_{1,0}\right)\left\{\langle \langle \psi \rangle \rangle \left[2 \lambda a_{0}\left\langle\left\langle f_{-}\right\rangle\right\rangle\langle\langle\psi \log (1-y)\rangle\rangle\right.\right. \\
+ & \left.\left.2 \lambda \lambda_{2,0}\left\langle\left\langle f_{-}\right\rangle\right\rangle\langle\langle\psi\rangle\rangle+a_{0}^{2}\left\langle\left\langle f_{-}\right\rangle\right\rangle\langle\langle\psi\rangle\rangle\right]+a_{0}\left\langle\left\langle f_{-}\right\rangle\right\rangle\left\langle\left\langle\psi^{2}\right\rangle\right\rangle\right\},
\end{aligned}
$$

where the reader will recall that $\lambda_{2,2}=\lambda / 2$, the other propagator coefficients being given after Eq. (B.2).

The non-universal terms at this order are collected at the end of the right-hand side of Eq. (B.2). Unlike the second order in $\delta$, at $O\left(\delta^{3}\right)$ there are non-universal terms proportional to $\log n$ appearing. It can be shown by explicit computation that up to $O\left(\delta^{4}\right)$, all such terms come from decaying power laws in $n$, which in addition vanish when $\left\langle\left\langle f_{-}\right\rangle\right\rangle \rightarrow 0$. Thus these non-universal terms survive neither in the limit $n \rightarrow \infty$ nor in the limit $q \rightarrow \infty$.

We have also performed the $O\left(\delta^{4}\right)$ computation; we do not show the diagrams. In Eq. (B.4) below, we organize the result into four parts: (universal) terms multiplying the third-order propagator $\lambda_{3,3} \log ^{3} n+\lambda_{3,2} \log ^{2} n+\lambda_{3,1} \log n+\lambda_{3,0}$, universal terms multiplying the second-order propagator $\lambda_{2,2} \log ^{2} n+\lambda_{2,1} \log n+\lambda_{2,0}$, non-universal terms multiplying this propagator, and terms both universal and non-universal multiplying the first order propagator $\lambda_{1,1} \log n+\lambda_{1,0}$, which we omit. The universal results allow the reader to check our re-summation in section 3 . The non-universal terms allow the reader to check our assertion that such terms resum to simple decaying power laws in $n$. 
$\langle\log \mathcal{Z}\rangle_{4}=\left(\lambda_{3,3} \log ^{3} n+\lambda_{3,2} \log ^{2} n+\lambda_{3,1} \log n+\lambda_{3,0}\right)$

$$
\begin{aligned}
& \cdot\left[-2 \lambda^{2}\left\langle\left\langle f_{-}\right\rangle\right\rangle^{2}\langle\langle\psi\rangle\rangle\left(\lambda\langle\langle\psi \log (1-y)\rangle\rangle+\lambda^{\prime}\langle\langle\psi\rangle\rangle\right)-\lambda^{2}\left\langle\left\langle f_{-}\right\rangle\right\rangle^{2}\left\langle\left\langle\psi^{2}\right\rangle\right\rangle\right] \\
& +\left(\lambda_{2,2} \log ^{2} n+\lambda_{2,1} \log n+\lambda_{2,0}\right) \\
& \text {. }\left\{2 \lambda^{3}\langle\langle\psi\rangle\rangle\left[\left\langle f_{-}\right\rangle\right\rangle\langle\langle\psi\rangle\rangle\left\langle\left\langle f_{-} \log (1-y) \log \frac{y}{1-y}\right\rangle\right\rangle\right. \\
& +\langle\langle\psi\rangle\rangle\left\langle\left\langle f_{-} \log \frac{y}{1-y}\right\rangle\right\rangle\langle\langle\psi \log (1-y)\rangle\rangle \\
& -5\left\langle\left\langle f_{-}\right\rangle\right\rangle\left\langle\left\langle f_{-} \log (1-y)\right\rangle\right\rangle\langle\langle\psi \log (1-y)\rangle\rangle \\
& \left.+\left\langle\left\langle f_{-}\right\rangle\right\rangle\left\langle\left\langle f_{-} \log y\right\rangle\right\rangle\langle\langle\psi \log (1-y)\rangle\rangle\right] \\
& +\lambda^{2} \lambda_{3,2}\langle\langle\psi\rangle\rangle^{3}\left\langle\left\langle f_{-} \log \frac{y}{1-y}\right\rangle\right\rangle \\
& +\lambda^{2} \lambda^{\prime}\langle\langle\psi\rangle\rangle\left[\langle\langle\psi\rangle\rangle^{2}\left\langle\left\langle f_{-} \log \frac{y}{1-y}\right\rangle\right\rangle\right. \\
& +4\left\langle\left\langle f_{-}\right\rangle\right\rangle\langle\langle\psi\rangle\rangle\left\langle\left\langle f_{-} \log \frac{y}{1-y}\right\rangle\right\rangle-4\left\langle\left\langle f_{-}\right\rangle\right\rangle^{2}\langle\langle\psi \log (1-y)\rangle\rangle \\
& \left.-8\left\langle\left\langle f_{-}\right\rangle\right\rangle\langle\langle\psi\rangle\rangle\left\langle\left\langle f_{-} \log (1-y)\right\rangle\right\rangle\right]-4 \lambda \lambda^{\prime 2}\left\langle\left\langle f_{-}\right\rangle\right\rangle^{2}\langle\langle\psi\rangle\rangle^{2} \\
& +\lambda^{2}\left[2\left\langle\left\langle f_{-}\right\rangle\right\rangle\langle\langle\psi\rangle\rangle\left\langle\left\langle f_{-} \psi \log \frac{y}{1-y}\right\rangle\right\rangle\right. \\
& -\left\langle\left\langle f_{-}\right\rangle\right\rangle\langle\langle\psi\rangle\rangle\left\langle\left\langle\psi^{2} \log (1-y)\right\rangle\right\rangle+\langle\langle\psi\rangle\rangle\left\langle\left\langle\psi^{2}\right\rangle\right\rangle\left\langle\left\langle f_{-} \log \frac{y}{1-y}\right\rangle\right\rangle \\
& -\langle\langle\psi \log (1-y)\rangle\rangle\left(\left\langle\left\langle f_{-}\right\rangle\right\rangle\left\langle\left\langle\psi^{2}\right\rangle\right\rangle+2\langle\langle\psi\rangle\rangle\left\langle\left\langle f_{-} \psi\right\rangle\right\rangle+\langle\langle\psi\rangle\rangle\left\langle\left\langle f_{-}^{2}\right\rangle\right\rangle\right) \\
& \left.-4\left\langle\left\langle f_{-}\right\rangle\right\rangle\left\langle\left\langle\psi^{2}\right\rangle\right\rangle\left\langle\left\langle f_{-} \log (1-y)\right\rangle\right\rangle\right] \\
& -\lambda \lambda^{\prime}\left(2\left\langle\left\langle f_{-}\right\rangle\right\rangle\langle\langle\psi\rangle\rangle\left\langle\left\langle\psi^{2}\right\rangle\right\rangle+2\left\langle\left\langle f_{-}\right\rangle\right\rangle^{2}\left\langle\left\langle\psi^{2}\right\rangle\right\rangle\right. \\
& \left.+2\langle\langle\psi\rangle\rangle^{2}\left\langle\left\langle f_{-} g_{+}\right\rangle\right\rangle+3\langle\langle\psi\rangle\rangle^{2}\left\langle\left\langle f_{-}^{2}\right\rangle\right\rangle\right) \\
& \left.-\lambda\left(\left\langle\left\langle f_{-} g_{+}\right\rangle\right\rangle\left\langle\left\langle\psi^{2}\right\rangle\right\rangle+\frac{3}{2}\left\langle\left\langle f_{-}^{2}\right\rangle\right\rangle\left\langle\left\langle\psi^{2}\right\rangle\right\rangle+\left\langle\left\langle f_{-}\right\rangle\right\rangle\left\langle\left\langle\psi^{3}\right\rangle\right\rangle\right)\right\} \\
& +\left(\lambda_{2,2} \log ^{2} n+\lambda_{2,1} \log n+\lambda_{2,0}\right) \\
& \cdot\left[-4 \lambda^{2} a_{0}\left\langle\left\langle f_{-}\right\rangle\right\rangle^{2}\langle\langle\psi\rangle\rangle\langle\langle\psi \log (1-y)\rangle\rangle-2 \lambda a_{0}^{2}\left\langle\left\langle f_{-}\right\rangle\right\rangle^{2}\langle\langle\psi\rangle\rangle^{2}\right. \\
& \left.-4 \lambda^{2} \lambda_{2,0}\left\langle\left\langle f_{-}\right\rangle\right\rangle^{2}\langle\langle\psi\rangle\rangle^{2}-2 \lambda a_{0}\left\langle\left\langle f_{-}\right\rangle\right\rangle^{2}\left\langle\left\langle\psi^{2}\right\rangle\right\rangle\right] \\
& +\left(\lambda_{1,1} \log n+\lambda_{1,0}\right) \cdot(\cdots) \text {. }
\end{aligned}
$$




\section{Appendix C: Some useful sums}

Our starting point is the standard exponential sum:

$$
\sum_{L=0}^{\infty} \frac{x^{L}}{L !}=\exp (x)
$$

and a modified version thereof

$$
\sum_{L=0}^{\infty} \sum_{l=0}^{L}\left(\begin{array}{l}
L \\
l
\end{array}\right) \frac{x^{L}}{L !}=\exp (2 x)
$$

Now consider the sum appearing in Eq. (3.10), which has the form

$$
\mathcal{S}^{\prime}(x, y)=\sum_{l_{1}=0}^{\infty} \sum_{l_{2}=1}^{\infty} \sum_{l=0}^{l_{2}} \sum_{m=0}^{\infty}\left(\begin{array}{c}
L-l \\
l_{1}
\end{array}\right) \frac{(m+l) !}{m ! l !} \frac{1}{(m+L+1) !} x^{m} y^{L}
$$

where $L=l_{1}+l_{2}$. It is convenient to add and subtract the term $l_{2}=0$ in Eq. (C.3), and set

$$
\mathcal{S}^{\prime}(x, y)=\mathcal{S}(x, y)-\mathcal{S}_{0}(x, y)
$$

with

$$
\mathcal{S}(x, y)=\sum_{L=0}^{\infty} \sum_{l_{1}=0}^{L} \sum_{l=0}^{L-l_{1}} \sum_{m=0}^{\infty}\left(\begin{array}{c}
L-l \\
l_{1}
\end{array}\right) \frac{(m+l) !}{m ! l !} \frac{1}{(m+L+1) !} x^{m} y^{L} .
$$

and

$$
\mathcal{S}_{0}(x, y)=\sum_{m=0}^{\infty} \sum_{l_{1}=0}^{\infty} \frac{1}{\left(m+l_{1}+1\right) !} x^{m} y^{l_{1}}
$$

In order to compute $\mathcal{S}(x, y)$ from Eq. (C.5) we first alter the order of summation

$$
\sum_{l_{1}=0}^{L} \sum_{l=0}^{L-l_{1}} \rightarrow \sum_{l=0}^{L} \sum_{l_{1}=0}^{L-l}
$$

leading immediately to 


$$
\mathcal{S}(x, y)=\sum_{L=0}^{\infty} \sum_{l=0}^{L} \sum_{m=0}^{\infty} 2^{-l} \frac{(m+l) !}{m ! l !} \frac{1}{(m+L+1) !} x^{m}(2 y)^{L} .
$$

To perform the remaining sums, we change variables to $N=m+L, n=m+l$, and rewrite the sums as

$$
\sum_{L=0}^{\infty} \sum_{l=0}^{L} \sum_{m=0}^{\infty} \rightarrow \sum_{N=0}^{\infty} \sum_{n=0}^{N} \sum_{m=0}^{n}
$$

We now have

$$
\mathcal{S}(x, y)=\sum_{N=0}^{\infty} \sum_{n=0}^{N} \sum_{m=0}^{n}\left(\begin{array}{c}
n \\
m
\end{array}\right) \frac{1}{(N+1) !} 2^{m-n} x^{m}(2 y)^{N-m} .
$$

The remaining sums are now elementary. Summing over $m$, we obtain

$$
\mathcal{S}(x, y)=\sum_{N=0}^{\infty} \sum_{n=0}^{N} \frac{1}{(N+1) !}\left(1+\frac{x}{y}\right)^{n} 2^{-n}(2 y)^{N},
$$

and summing over $n$,

$$
\mathcal{S}(x, y)=\sum_{N=0}^{\infty} \frac{(2 y)^{N}}{(N+1) !} \frac{\left(1-\left[\frac{1}{2}\left(1+\frac{x}{y}\right)\right]^{N+1}\right)}{\left(1-\frac{1}{2}\left(1+\frac{x}{y}\right)\right)} .
$$

Recognizing that

$$
\sum_{N=0}^{\infty} \frac{x^{N}}{(N+1) !}=\frac{\exp (x)-1}{x}
$$

we obtain our final result:

$$
\begin{aligned}
\mathcal{S}(x, y) & =\frac{1}{\left(1-\frac{1}{2}\left(1+\frac{x}{y}\right)\right)}\left\{\frac{\exp (2 y)-1}{2 y}-\frac{1}{2}\left(1+\frac{x}{y}\right) \frac{\exp \left(2 y \cdot \frac{1}{2}\left(1+\frac{x}{y}\right)\right)-1}{2 y \cdot \frac{1}{2}\left(1+\frac{x}{y}\right)}\right\} \\
& =e^{y}\left(\frac{e^{y}-e^{x}}{y-x}\right) .
\end{aligned}
$$


A similar but simpler algebra yields the sum $\mathcal{S}_{0}(x, y)$ from Eq. (C.6),

$$
\mathcal{S}_{0}(x, y)=\frac{e^{y}-e^{x}}{y-x}
$$

so that

$$
\mathcal{S}^{\prime}(x, y)=\left(e^{y}-1\right)\left(\frac{e^{y}-e^{x}}{y-x}\right)
$$

This result illustrates the necessary tricks for performing the types of sums appearing in this paper. To give a further example, the sum appearing in Eq. (3.9) is of the form

$$
\mathcal{S}^{\prime \prime}(x, y)=\sum_{l_{1}=0}^{\infty} \sum_{l_{2}=0}^{\infty} \sum_{m_{1}=0}^{\infty} \sum_{m_{2}=0}^{\infty} \sum_{l=0}^{l_{2}}\left(\begin{array}{c}
L-l \\
l_{1}
\end{array}\right) \frac{(M+l+1) !}{m_{1} !\left(m_{2}+l+1\right) !} \frac{1}{(M+L+2) !} x^{L} y^{M}
$$

with $L=l_{1}+l_{2}$ and $M=m_{1}+m_{2}$. Once again,

$$
\sum_{l_{1}=0}^{\infty} \sum_{l_{2}=0}^{\infty} \sum_{l=0}^{l_{2}} \rightarrow \sum_{L=0}^{\infty} \sum_{l=0}^{L} \sum_{l_{1}=0}^{L-l}
$$

so that

$$
\mathcal{S}^{\prime \prime}(x, y)=\sum_{m_{1}=0}^{\infty} \sum_{m_{2}=0}^{\infty} \sum_{L=0}^{\infty} \sum_{l=0}^{L} 2^{L-l} \frac{(M+l+1) !}{m_{1} !\left(m_{2}+l+1\right) !} \frac{1}{(M+L+2) !} x^{L} y^{M} .
$$

In addition to $M=m_{1}+m_{2}$ and $L=l_{1}+l_{2}$, we take $N=M+L$, and $n=M+l$. We then rearrange the sums

$$
\sum_{m_{1}=0}^{\infty} \sum_{m_{2}=0}^{\infty} \sum_{L=0}^{\infty} \sum_{l=0}^{L} \rightarrow \sum_{N=0}^{\infty} \sum_{n=0}^{N} \sum_{m_{1}=0}^{n} \sum_{M=m_{1}}^{n}
$$

we find that the sums can now be performed to yield 


$$
\mathcal{S}^{\prime \prime}(x, y)=\frac{1}{2}\left(\frac{e^{x}-e^{y}}{x-y}\right)^{2}
$$

The other sums appearing in section 3 can all be performed using this repertoire of tricks.

\section{Appendix D: Computation of $\log \langle\mathcal{Z}\rangle$}

Although, as we have seen, the computation of $\langle\log \mathcal{Z}\rangle$ is quite intricate, the computation of $\langle\mathcal{Z}\rangle$ is by contrast straightforward. This is quite useful, since we have seen that the terms in $\langle\log \mathcal{Z}\rangle$ that are $\propto \log n$ as $n \rightarrow \infty$ are identical to $\log \langle\mathcal{Z}\rangle$.

Consider the initial branching point in the cluster. At this point, we can write the relation

$$
\langle\mathcal{Z}(q, \sigma ; n)\rangle=\int_{0}^{\infty} d \epsilon \rho(\epsilon)\left\{f_{-}(\epsilon n)\langle\mathcal{Z}(q, \sigma ; y(\epsilon n) n)\rangle+f_{+}(\epsilon n)\langle\mathcal{Z}(q, \sigma ;(1-y(\epsilon n)) n)\rangle\right\}
$$

where the averages inside the integral on the right-hand side now do not include the first average over $\epsilon$. In Ref. 11, we showed how this integral equation can be expanded into differential equations of increasing order, whose solution determines the annealed dimensions $\sigma_{\mathcal{A}}(q)$. For general $\sigma$ these differential equations have solutions of the form $\langle\mathcal{Z}\rangle=n^{\mu(q, \sigma)}$. Using this Ansatz in Eq. (D.1), we obtain

$$
n^{\mu}=\int_{0}^{\infty} d \epsilon \rho(\epsilon)\left\{f_{-}(\epsilon n)[y(\epsilon n) n]^{\mu}+f_{+}(\epsilon n)[(1-y(\epsilon n)) n]^{\mu}\right\}
$$

Factoring out $n^{\mu}$, substituting $f_{+} \equiv 1+g_{+}$, and then transforming to the $\eta \equiv \epsilon n$ variable, we see that

$$
\begin{aligned}
\int_{0}^{\infty} d \eta \eta^{\nu-1}\left\{f_{-}(\eta) y^{\mu}(\eta)+\left(1+g_{+}(\eta)\right)\right. & {\left.[1-y(\eta)]^{\mu}-1\right\} } \\
& \equiv\left\langle\left\langle f_{-} y^{\mu}+\left(1+g_{+}\right)(1-y)^{\mu}-1\right\rangle\right\rangle=0,
\end{aligned}
$$


which implicitly determines $\mu(q, \sigma)$. We can expand Eq. (D.3) so as to obtain $\mu$ order by order in $\delta$, the formal parameter that counts the number of powers of $f_{-}$or $g_{+}$. If $f_{-} \rightarrow 0$ and $g_{+} \rightarrow 0$, we see that $\mu \rightarrow 0$ as well. To implement the expansion, we write $y^{\mu} \equiv \exp (\mu \log y)$ and $(1-y)^{\mu}=\exp (\mu \log (1-y))$; we can then expand the exponentials order by order in $\mu$ in Eq. (D.3), thereby obtaining solutions for $\mu$ to any desired order in $\delta$. To second order in $\delta$, we can write

$$
\left\langle\left\langle\psi+\mu\left[\log (1-y)+g_{+} \log (1-y)+f_{-} \log y\right]+\frac{\mu^{2}}{2} \log ^{2}(1-y)\right\rangle\right\rangle+O\left(\delta^{3}\right)=0,
$$

which gives

$$
\mu=-\frac{\langle\langle\psi\rangle\rangle}{\langle\langle\log (1-y)\rangle\rangle}\left[1-\frac{\left\langle\left\langle f_{-} \log y+g_{+} \log (1-y)\right\rangle\right\rangle}{\langle\langle\log (1-y)\rangle\rangle}+\frac{\langle\langle\psi\rangle\rangle\left\langle\left\langle\log ^{2}(1-y)\right\rangle\right\rangle}{2\langle\langle\log (1-y)\rangle\rangle^{2}}\right]+O\left(\delta^{3}\right),
$$

or, recalling the definitions of $\lambda$ and $\lambda^{\prime}$ (Eqs. (A.5) and (A.10)),

$$
\mu=\lambda\langle\langle\psi\rangle\rangle\left[1+\lambda\left\langle\left\langle f_{-} \log y+g_{+} \log (1-y)\right\rangle\right\rangle+\lambda^{\prime}\langle\langle\psi\rangle\rangle+O\left(\delta^{2}\right)\right]
$$

which agrees with the term $\propto \log n$ in Eq. (3.43) above. Note that $\langle\langle\psi\rangle\rangle=0$ implies that $\mu=0$ to any order in $\delta$. Clearly terms of higher order in $\delta$ can be obtained from Eq. (D.3) with considerably less labor than by direct perturbative computation.

\section{Appendix E: $\delta$ expansion as an expansion in $q-1$}

The perturbation expansion derived above can be formally written in the following form:

$$
\langle\log \mathcal{Z}\rangle=\langle\langle\psi\rangle\rangle F_{1}(q, \sigma ; n)+\left\langle\left\langle\psi^{2}\right\rangle\right\rangle F_{2}(q, \sigma ; n)+\cdots,
$$

because the last vertex (at the far right) of any diagram is always averaged without any powers of $\log y$ or $\log (1-y)$, and can always be either $f_{-}, g_{+}$, or a product of factors of these two. Thus this last vertex can always be regarded as being of the form 
$\left\langle\left\langle\psi^{n}\right\rangle\right\rangle$. (This property is satisfied by Eqs. (3.43) and (B.1-4).) Recall the definition of $\psi$ :

$$
\psi(\eta ; q, \sigma)=f_{-}(\eta)+g_{+}(\eta)=\frac{x^{q}(\eta)}{y^{\sigma}(\eta)}+\frac{(1-x(\eta))^{q}}{(1-y(\eta))^{\sigma}}-1 .
$$

At $q=1$, for $\sigma=0$ we have $\psi=0$, which implies that both $\sigma_{\mathcal{A}}(q)$ and the apparent $\sigma_{\mathcal{P}}(q)$ are equal to zero. Thus it is clear that

$$
\psi\left(q, \sigma_{\mathcal{P}}(q) ; \eta\right)=O(q-1)
$$

Since by definition $\left\langle\left\langle\psi\left(q, \sigma_{\mathcal{A}}(q)\right)\right\rangle\right\rangle=0$ exactly, Eq. (E.1) together with (E.3) implies that $\sigma_{\mathcal{P}}(q)=\sigma_{\mathcal{A}}(q)+O\left((q-1)^{2}\right)$, with

$$
\left\langle\left\langle\psi\left(q, \sigma_{\mathcal{P}}(q)\right)\right\rangle\right\rangle=O\left((q-1)^{2}\right),
$$

and

$$
\left\langle\left\langle\psi^{2}\right\rangle\right\rangle=O\left((q-1)^{2}\right) .
$$




\section{References}

1. T.A. Witten, Jr. and L.M. Sander, Phys. Rev. Lett. 47, 1400 (1981).

2. P. Meakin, Phys. Rev. A 27, 1495 (1983).

3. P. Meakin, in Phase Transitions and Critical Phenomena, Vol. 12 eds. C. Domb and J. Lebowitz, (Academic Press, New York, 1988).

4. T. Vicsek, Fractal Growth Phenomena, (World Scientific, Singapore, 1989)

5. H. Gould, F. Family, and H.E. Stanley, Phys. Rev. Lett. 50, 686 (1983); T. Nagatani, Phys. Rev. A 36, 5812 (1987); J. Phys. A 20, L381 (1987); P. Barker and R.C. Ball, Phys. Rev. A 42, 6289 (1990).

6. X.R. Wang, Y. Shapir and M. Rubinstein, Phys. Rev. A 39, 5974 (1989); J. Phys. A 22, L507 (1989).

7. L. Pietronero, A. Erzan, and C.J.G. Evertsz, Phys. Rev. Lett. 61, 861 (1988); Physica A 151, 207 (1988).

8. P. Meakin, H.E. Stanley, A. Coniglio, and T.A. Witten, Jr., Phys. Rev. A 32, 2364 (1985); C. Amitrano, A. Coniglio, and F. di Liberto, Phys. Rev. Lett. 57, 1016 (1986); C. Amitrano, Phys. Rev. A 39, 6618 (1989).

9. T.C. Halsey, P. Meakin, and I. Procaccia, Phys. Rev. Lett. 56, 854 (1986); T.C. Halsey, Phys. Rev. Lett. 59, 2067 (1987); Phys. Rev. A 38, 4749 (1988).

10. Y. Hayakawa, S. Sato, and M. Matsushita, Phys. Rev. A 36, 1963 (1987); R.C. Ball and O. Rath Spivack, J. Phys. A 23, 5295 (1990).

11. T.C. Halsey and M. Leibig, Phys. Rev. A 46, 7792 (1992).

12. T.C. Halsey, Phys. Rev. Lett. 72, 1228 (1994).

13. R. Blumenfeld and A. Aharony, Phys. Rev. Lett. 62, 2977 (1989); R.C. Ball and R. Blumenfeld, Phys. Rev. A 44, 828 (1991); S. Schwarzer, J. Lee, A. Bunde, S. Havlin, H.E. Roman, and H.E. Stanley, 65, 603 (1990); B. Mandelbrot and C.J.G. Evertsz, Nature 348, 143 (1990); S. Schwarzer, J. Lee, S. Havlin, H.E. Stanley, and P. Meakin, Phys. Rev. A 431134 (1991).

14. B.B. Mandelbrot, H. Kaufman, A. Vespinani, I. Yekutieli, and C.H. Lam, Europhys. Lett. 29, 599 (1995).

15. P. Ossadnik, Physica A 195, 319 (1993). 
16. M. Muthukumar, Phys. Rev. Lett. 50, 839 (1983); S. Tolman and P. Meakin, Phys. Rev. A 40, 428 (1989).

17. R. Brady and R.C. Ball, Nature (London) 309, 225; J. Nittmann, G. Daccord, and H.E. Stanley, Nature (London) 314, 141 (1985); D. Kessler, J. Koplik, and H. Levine, Advances in Physics 37, 255 (1988).

18. P. Meakin, Phys. Rev. Lett. 51, 1119 (1983); M. Kolb, R. Botet, and R. Jullien, ibid, 1123; J. Nittmann, G. Daccord, and H.E. Stanley, Nature (London) 314, 141 (1985); P. Meakin, G. Li, L.M. Sander, H. Yan, F. Guinea, O. Pla, and E. Louis, in Disorder and Fracture, J.C. Charmet, S. Roux, and E. Guyon, eds. (Plenum Press, New York, 1990).

19. A. Renyi, Probability Theory (North-Holland, Amsterdam, 1970); B.B. Mandelbrot, Ann. Isr. Phys. Soc. 225 (1977); H.G.E. Hentschel and I. Procaccia, Physica 8D, 435 (1983); U. Frisch and G. Parisi, in Turbulence and Predictability in Geophysical Fluid Dynamics and Climate Dynamics, Proc. of Int. School of Physics "Enrico Fermi" LXXXVIII, eds. M. Ghil, R. Benzi, and G. Parisi (North-Holland, Amsterdam,1985) p. 84.

20. T.C. Halsey, M.H. Jensen, L.P. Kadanoff, I. Procaccia, and B. Shraiman, Phys. Rev. A 33, 1141(1986).

21. M.E. Cates and T.A. Witten, Phys. Rev. A 35, 1809 (1987); T.C. Halsey, in Fractals: Physical Origin and Properties, ed. L. Pietronero, (Plenum Publishing Co., London, 1989).

22. S. Redner, Am. J. Phys. 58, 267 (1990).

23. B.B. Mandelbrot, in Fractals: Physical Origin and Properties, ed. L. Pietronero, (Plenum Publishing Co., London, 1989); A.B. Chhabra and K.R. Sreenivasan, Phys. Rev. A 43, 1114 (1991).

24. T.C. Halsey, K. Honda, B. Duplantier, unpublished. 


\section{Table caption}

1. This table shows the orders of the terms divergent in $\log n$ appearing in the $\delta$ expansion for $\langle\log \mathcal{Z}\rangle$. A solid cross indicates that a term of that order in $\delta$ and $\log n$ appears; a dashed cross indicates that while such terms appear in some diagrams, they cancel between diagrams. The leading and sub-leading resummations discussed in section 3 correspond to sums down the diagonals of this table. 


\section{Figure captions}

1. A typical two dimensional DLA cluster, grown using off-lattice random walkers. There are approximately 35, 000 particles in this cluster (courtesy of M. Leibig).

2. Multifractal scaling functions $f(\alpha)$ for (a) a typical non-stochastic system, and (b) a typical stochastic system, with annealed averaging. Note the appearance of negative values of $f$ in the latter case. These functions are obtained by Legendre transformation of $\tau(q)$ or $\sigma(q) \propto \tau(q)$.

3. The unstable manifold for the dynamics of branch competition in "model Z". The two branches move along the indicated diagonal line until either $x=0$ or $x=1$. This corresponds to the complete screening of one or the other of the two sibling branches. From this point only the parameter $y$ reflecting the relative masses of the two branches changes.

4. The unstable manifold for the dynamics of branch competition for DLA in two dimensions, as calculated by a renormalization method in Ref. 12. Comparing with model Z, we see that screening is a more gradual process in DLA.

5. Two sibling elementary sub-branches. The last node in the branching cluster is indexed by $J$, has a total of $n_{J}$ descendants, and has a total growth probability of $p_{J}$. The stochastic variable controlling the branching at this node is $\epsilon_{J}$. The stronger descendant sub-branch has a number of particles $n_{e}=\left(1-y\left(\epsilon_{J} n_{J}\right)\right) n_{J}$, and a total growth probability $p_{e}=\left(1-x\left(\epsilon_{J} n_{J}\right)\right) p_{J}$

6. The sub-branch found at the bottom of the main branch, defined by taking at every node the stronger of the two paths down the branch. The index $j$ indexes the nodes along this main branch.

7. The terms of first order in $\delta$ containing one factor of $f$-arise from the elementary sub-branches at the end of the "first-order" sidebranches off of the main branch. The terms of first order in $\delta$ containing one factor of $g_{+}$arise from corrections to the partition function contribution coming from the elementary sub-branch at the end of the main branch.

8. Diagrams at $O(\delta)$ in perturbation theory. The solid circle indicates an $f_{-}$vertex, and the open circle indicates a $g_{+}$vertex. A solid and an open circle connected 
by a short vertical line indicate a vertex of $\psi=f_{-}+g_{+}$. A horizontal segment indicates a branch over which $n^{-\nu}$ is summed, possibly with a logarithmic factor in $n$.

9. A diagram at $O\left(\delta^{2}\right)$ that is $\propto g_{+}^{2}$. This diagram comes from $\langle\mathcal{Z}-1\rangle$. The two horizontal bars indicate the two propagator sums. Note that including a factor of $g_{+}$does not lead one onto a weaker side-branch.

10. A diagram at $O\left(\delta^{2}\right)$ that is $\propto f_{-}^{2}$. This diagram comes from $\langle\mathcal{Z}-1\rangle$. The two horizontal bars indicate the two propagator sums. The vertical bar indicates that the rightmost $f_{-}$vertex is off of the main branch. Taking a factor of $f_{-}$in a diagram arising from $\langle\mathcal{Z}-1\rangle$ always moves one onto a weaker sidebranch.

11. Mixed terms $\propto f_{-} \cdot g_{+}$arising from $\langle\mathcal{Z}-1\rangle$. These diagrams are $O\left(\delta^{2}\right)$.

12. All terms of $O\left(\delta^{2}\right)$ arising from $-(1 / 2)\left\langle(\mathcal{Z}-1)^{2}\right\rangle$. Note the symmetry factors.

13. All diagrams containing only one propagator in the full expression for $\langle\log \mathcal{Z}\rangle$. Note that the joined vertices indicate factors of $f_{-}$or $g_{+}$averaged together, e.g. $\left\langle\left\langle f_{-} g_{+}\right\rangle\right\rangle$for the last diagram. The sum of these diagrams gives the "onepropagator" approximation $\langle\log \mathcal{Z}\rangle_{1 p}$.

14. A typical contribution to $\langle\mathcal{Z}-1\rangle$. At leading order, the leading logarithm in each propagator is taken. Each vertical segment indicates a move to a side-branch.

15. A typical contribution to $\left\langle(\mathcal{Z}-1)^{2}\right\rangle$, arising from a product of a diagram with $l_{1}$ factors of $g_{+}$upstream from the first factor of $f_{-}$, with $m_{1}$ factors of $\psi$ off of the main branch, with a diagram with $l_{2}$ factors of $g_{+}$upstream from the first factor of $f_{-}$, with $m_{2}$ factors of $\psi$ off of the main branch. For simplicity, we have drawn all factors of $\psi$ along the same branch; terms involving more than one factor of $f_{-}$will properly have further vertical segments. (Note that we use "upstream" to mean closer to the root, at the left, not closer to the elementary sub-branches.)

16. One term arising from the product indicated in Figure 15. Defining $L=l_{1}+l_{2}$, we have $L-l \geq l_{1}$ factors of $g_{+}$upstream of the first factor of $f_{-}$, followed by $l \leq l_{2}$ factors of $g_{+}$, and then the second factor of $f_{-}$. The diagram includes $m_{1}$ factors of $\psi$ coming off of the first $f_{-}$vertex, and $m_{2}$ factors of $\psi$ coming off of the second $f_{-}$vertex.

17. This diagram indicates a term in the product indicated in Figure 15 that is $\propto$ 
$\left\langle\left\langle f_{-}^{2}\right\rangle\right\rangle$. Now all $L=l_{1}+l_{2}$ factors of $g_{+}$are to the left of the vertex containing the two factors of $f_{-}$averaged together. Note that at the sub-leading order, which we are considering here, the two separate side-branches emanating from these two factors of $f_{-}$(one containing $m_{1}$ factors of $\psi$, and the other containing $m_{2}$ factors of $\psi$ ) can be treated as independent.

18. This diagram indicates a term $\propto\left\langle\left\langle f_{-}^{2}\right\rangle\right\rangle$ arising from $\left.\left\langle(\mathcal{Z}-1)^{3}\right\rangle\right\rangle$. At sub-leading order, the factor of $\mathcal{Z}-1$ whose first $f_{-}$vertex appears separately can be averaged independently of the other two factors of $\mathcal{Z}-1$.

19. At sub-leading order, terms $\propto\left\langle\left\langle f_{-} \log y\right\rangle\right\rangle$ can be obtained by averaging a single factor of $\mathcal{Z}-1$; a typical diagram is shown. The arrows indicate the $f_{-}$vertices, each of which is a potential source of a factor of $\left\langle\left\langle f_{-} \log y\right\rangle\right\rangle$ except the last, which has no propagator to the right to provide a factor of $\log y$.

20. At sub-leading order, terms $\propto\left\langle\left\langle f_{-} \log (1-y)\right\rangle\right\rangle$ require at least two factors of $\mathcal{Z}-1$. This diagram arises from a product of the form shown. The arrow in the diagram corresponding to a single factor of $\mathcal{Z}-1$ indicates the vertex (with a factor of one rather than a factor of $f_{-}$or $\left.g_{+}\right)$at which the term $\log (1-y)$, arising from the propagator, is taken. To the right of this arrow, it is not necessary to keep track of whether vertices are $f_{-}$or $g_{+}$, provided at least one such vertex appears. In the combination of the two factors of $\mathcal{Z}-1$, this $\log (1-y)$ is averaged with a factor of $f_{-}$contributed by the other partition function.

21. Numerical results for $\left\langle\log \sum_{i} p_{i}^{q}\right\rangle$ vs. $\log n$ for a Monte Carlo realization of model $\mathrm{Z}$ random branched growth, for $q=2$ and $q=5$. We used a parameter $\nu=0.6$ to specify model Z. The linear slope indicates apparent multifractality, the value of the slope giving the apparent dimensions $\sigma_{\mathcal{P}}(q)$.

22. (a) The apparent dimensions $\sigma_{\mathcal{P}}(q)$ vs. $q$ for $0<q<10$, and (b) the Legendre transform of this function, $f(\alpha)$, computed numerically for model $\mathrm{Z}$ with $\nu=0.6$. No negative values of $f$ appear. In addition, since we compute $\sigma_{\mathcal{A}}(q)$ only for $q>0$, we show only the left side of the multifractal spectrum. Note that model $\mathrm{Z}$ has the pathological feature that $\lim _{q \rightarrow 0} \sigma(q) \neq-1$, due to the fact that much of the cluster surface has growth probability zero.

23. $\sigma$ vs. $q$ for model $\mathrm{Z}$ with $\nu=0.6$ computed in various manners. (a) solid line: 
numerical results, as in Figure 22(a); (b) dashed line: annealed dimensions $\sigma_{\mathcal{A}}(q)$; these are also the apparent dimensions $\sigma_{\mathcal{P}}(q)$ at $O(\delta)$; (c) dot-dashed line: apparent dimensions, computed to $O\left(\delta^{2}\right)$, and (d) dotted line: the one-propagator result for $\sigma(q)$, from Eq. (2.37). We note that the computation for apparent dimensions gives excellent results for $q<5$, but fails at higher values of $q$. The one-propagator result gives qualitatively correct results over the entire range of $q$.

24. Diagrams at $O(\delta)$ and $O\left(\delta^{2}\right)$. These correspond to the terms appearing in the text in Eqs. (B.1-2). They also summarize Figs. (8-12).

25. Diagrams at $O\left(\delta^{3}\right)$. These correspond to the terms appearing in Eq. (B.3). 


\section{TABLE I}

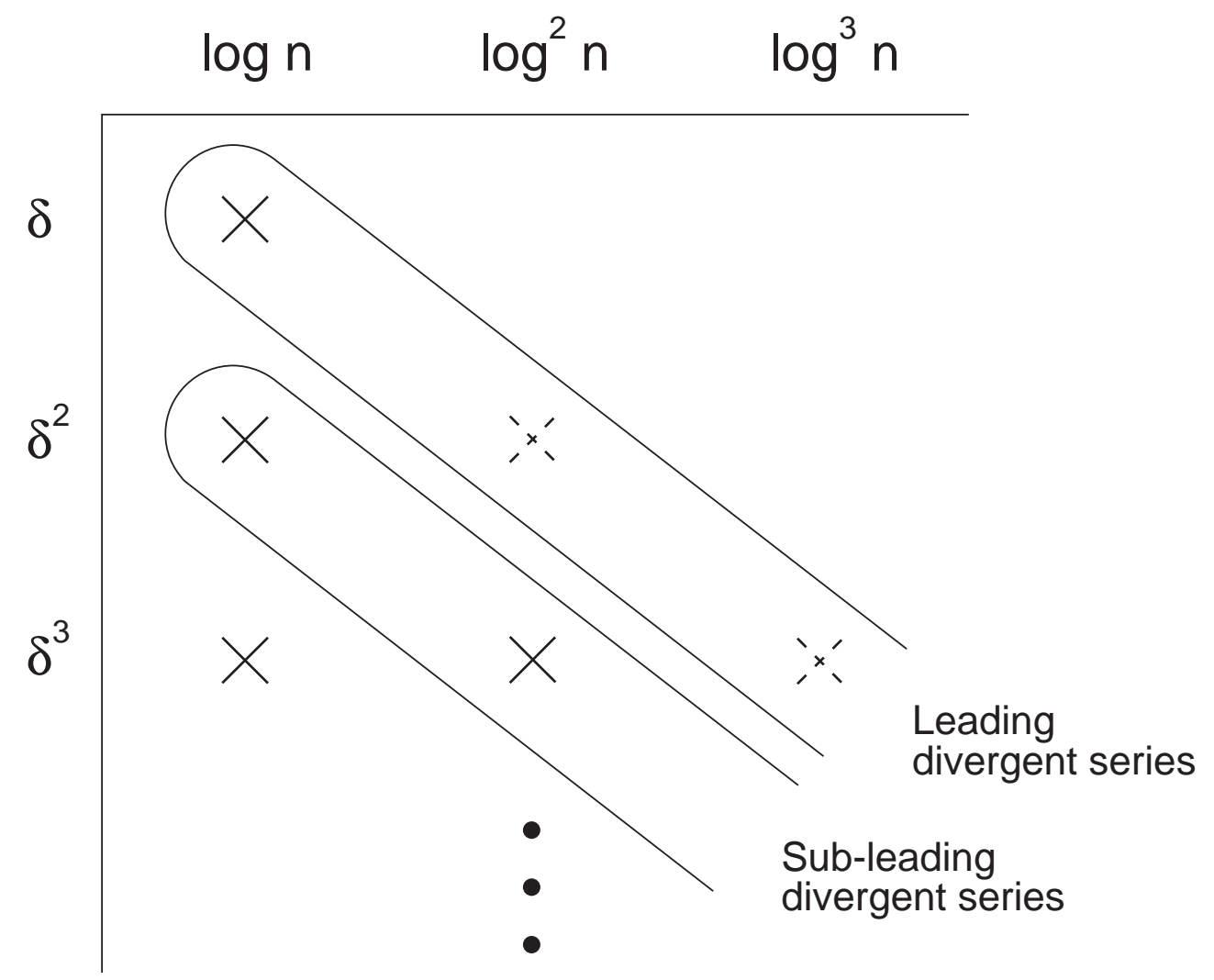


FIGURE 2(a)

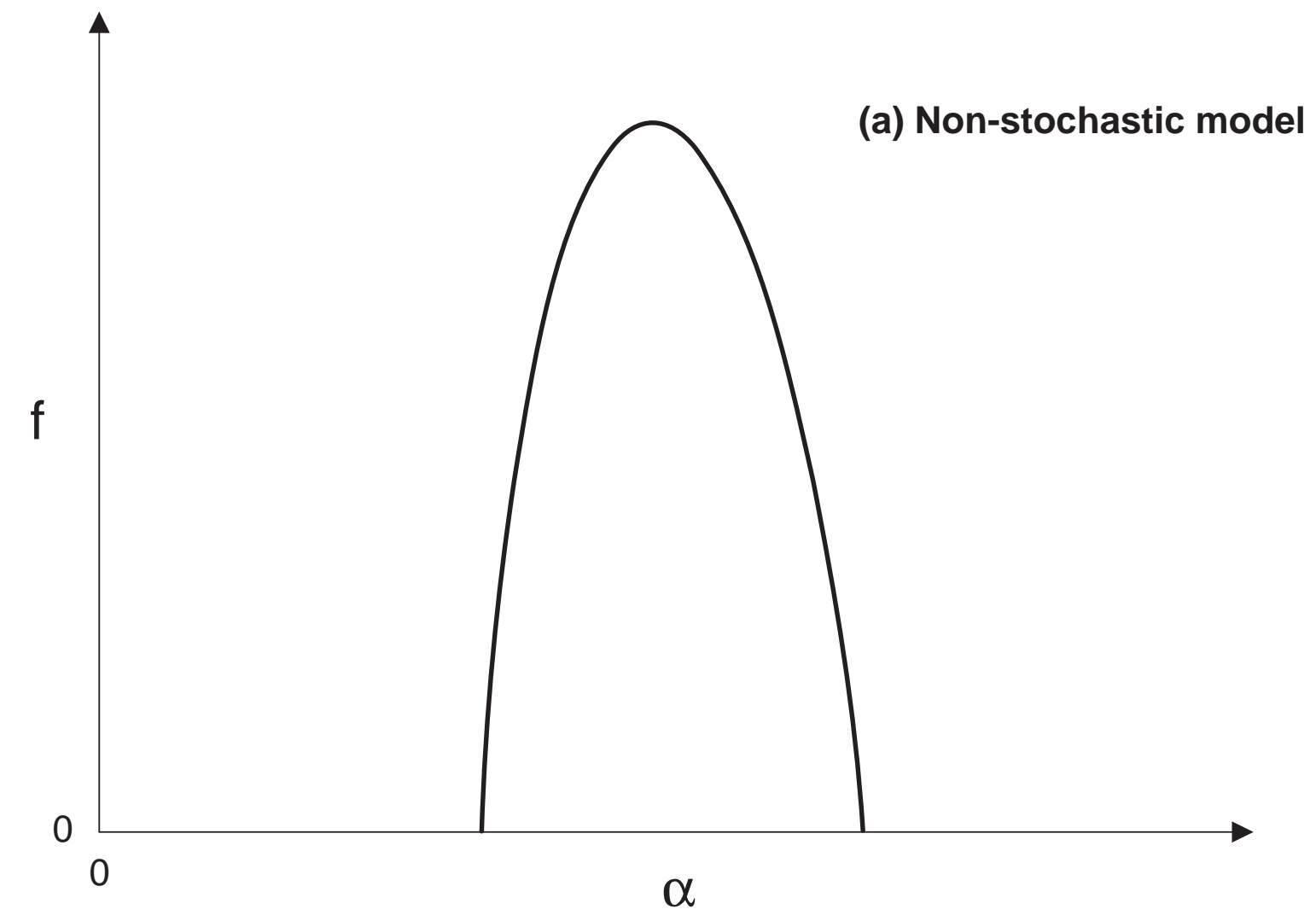


FIGURE 2(b)

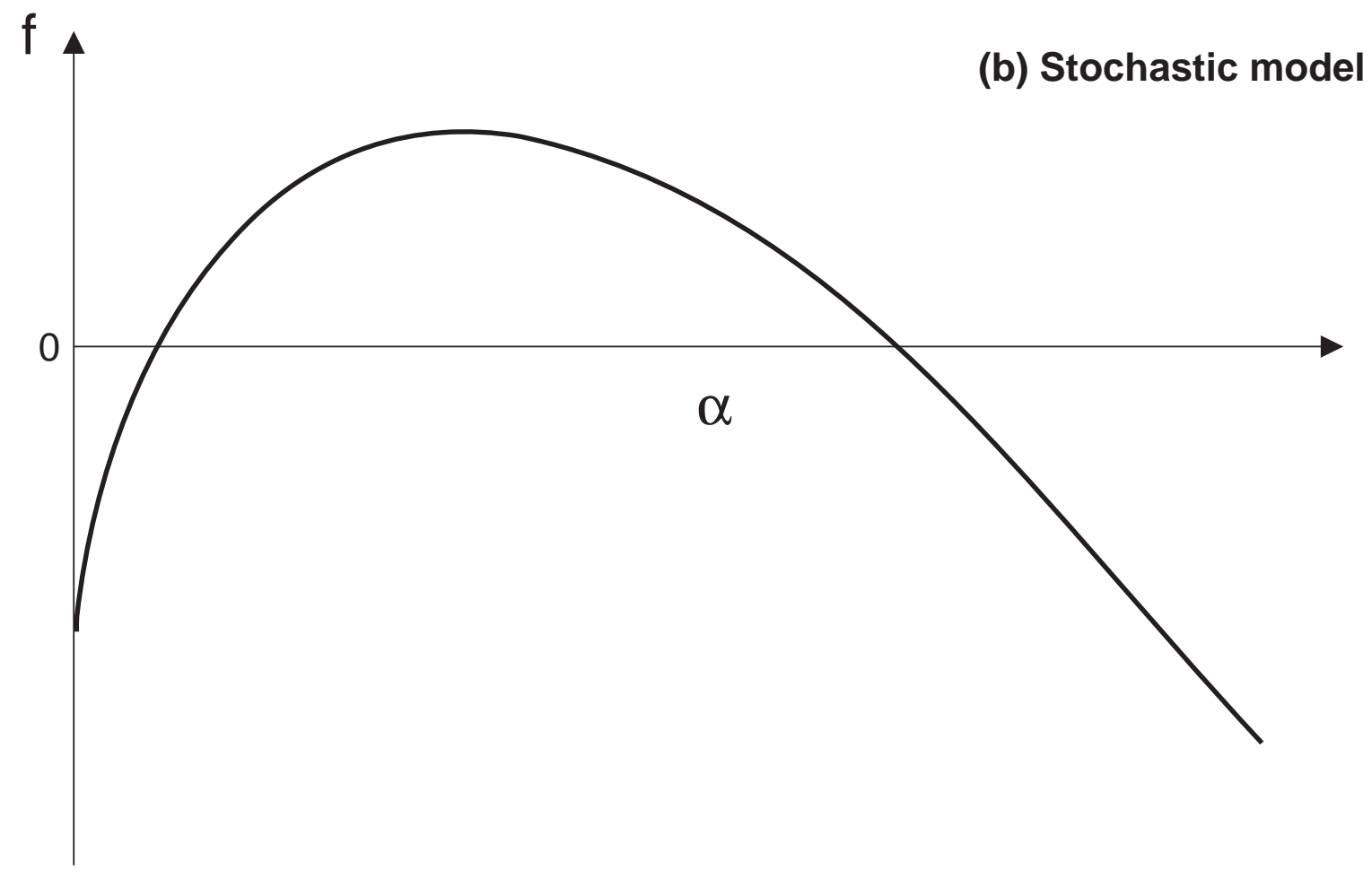


FIGURE 3

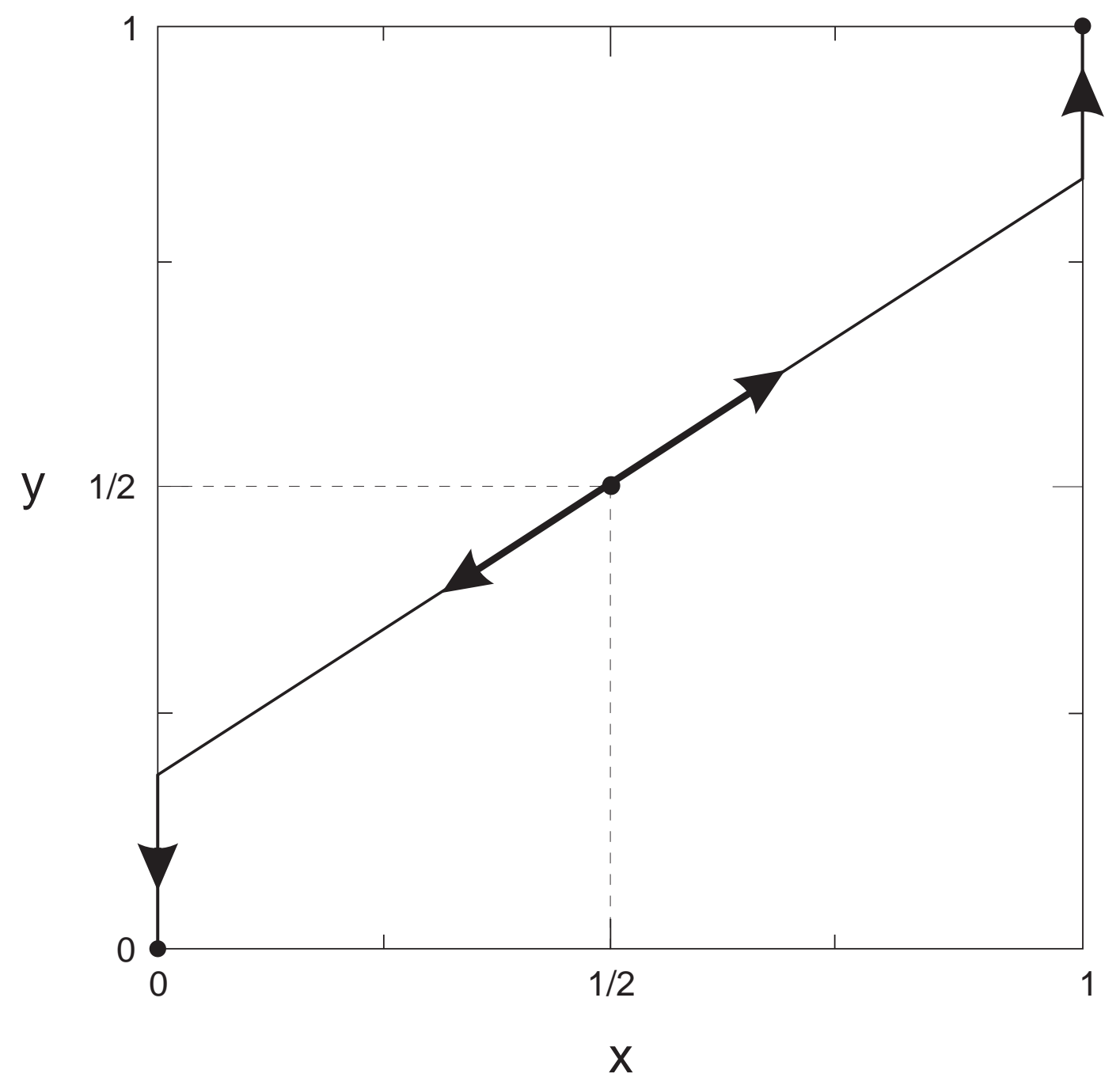


FIGURE 4

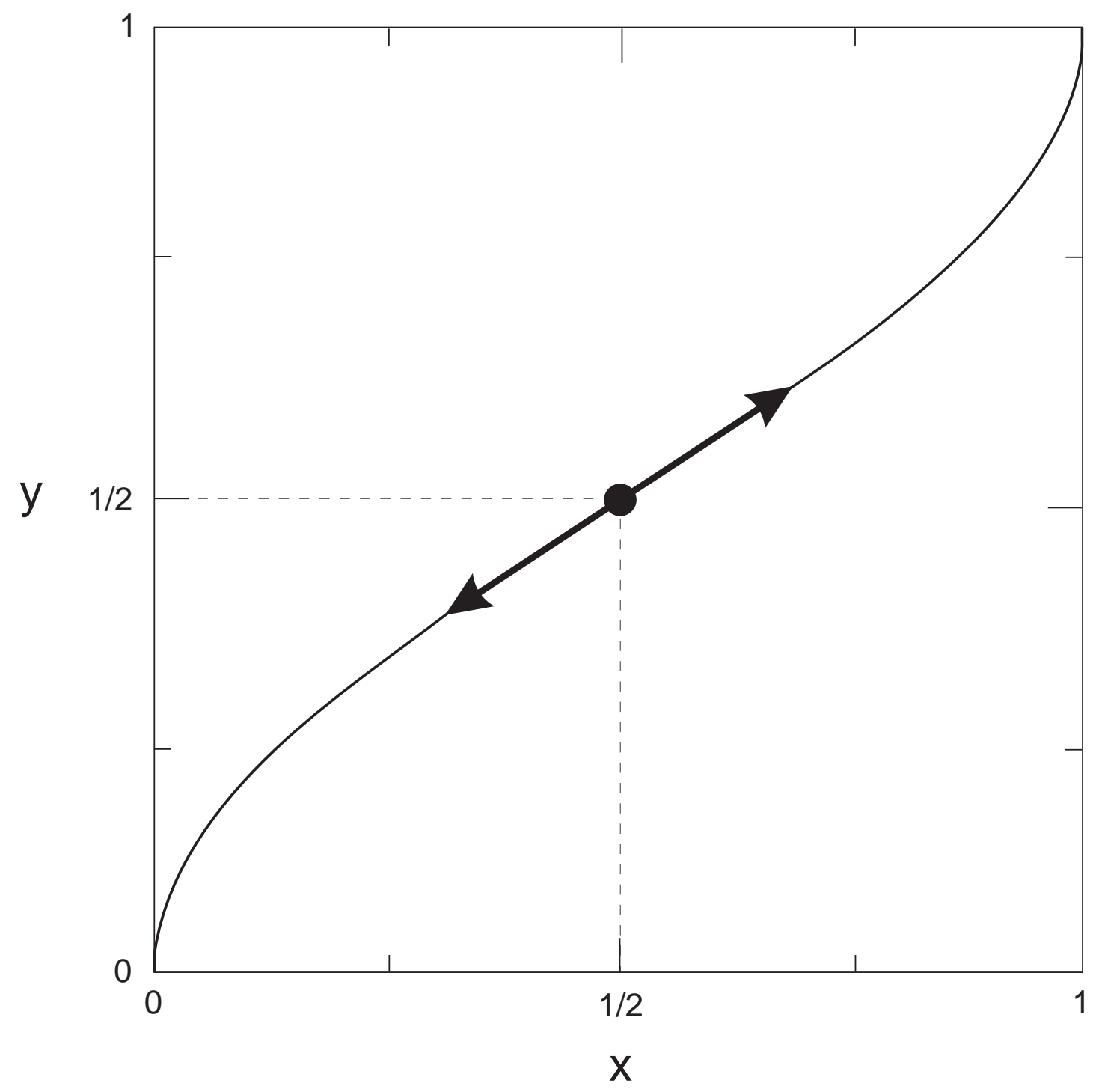


FIGURE 5

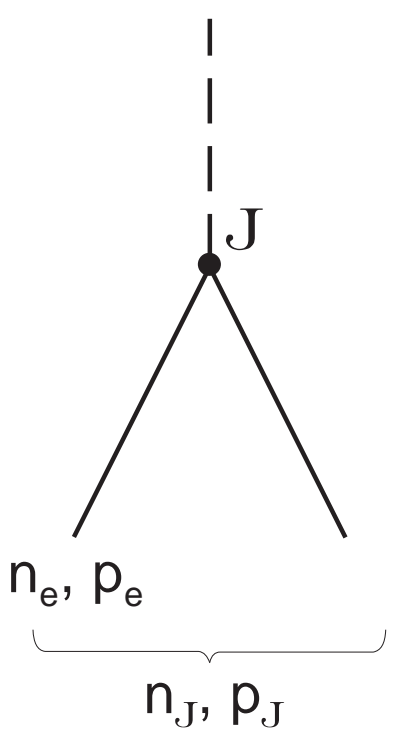

FIGURE 6

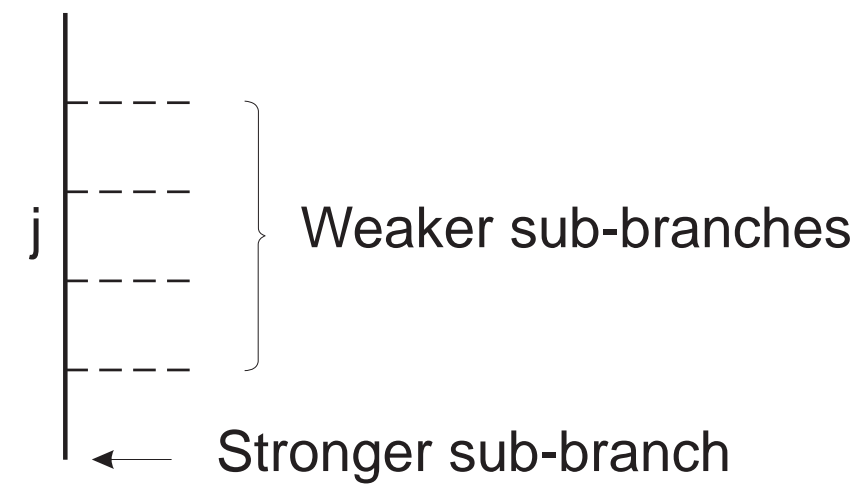

FIGURE 7

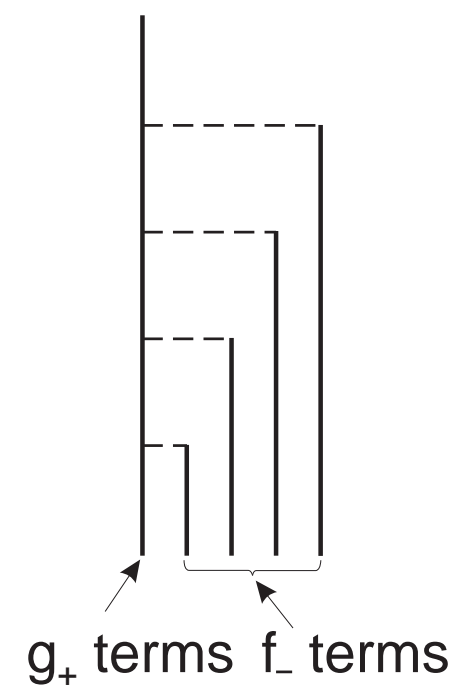


FIGURE 8

$\bullet+\longrightarrow \equiv$

FIGURE 9

FIGURE 10

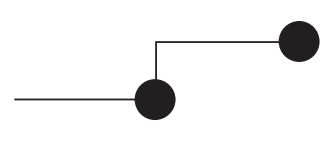

FIGURE 11

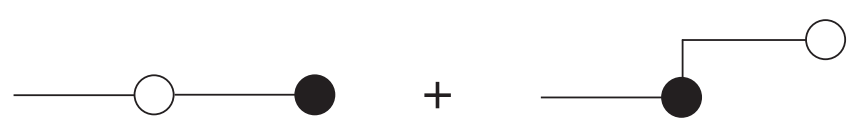

FIGURE 12

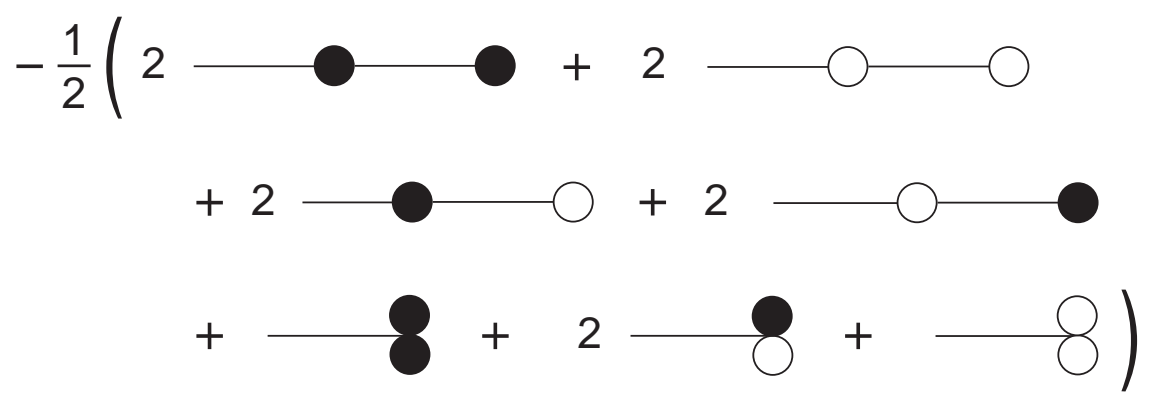


FIGURE 13

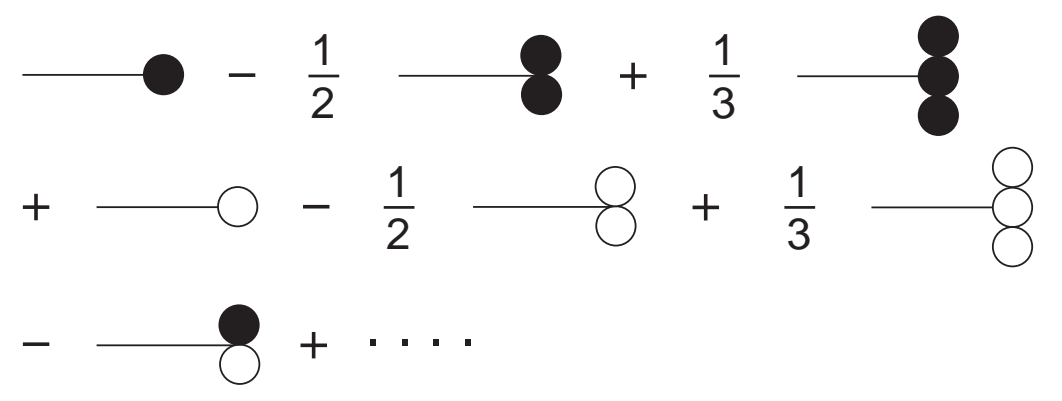

FIGURE 14

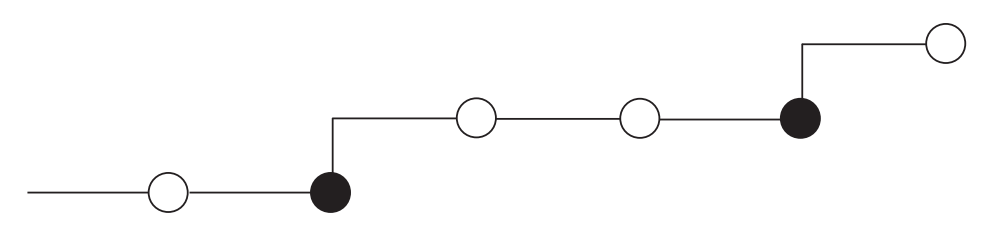

FIGURE 15
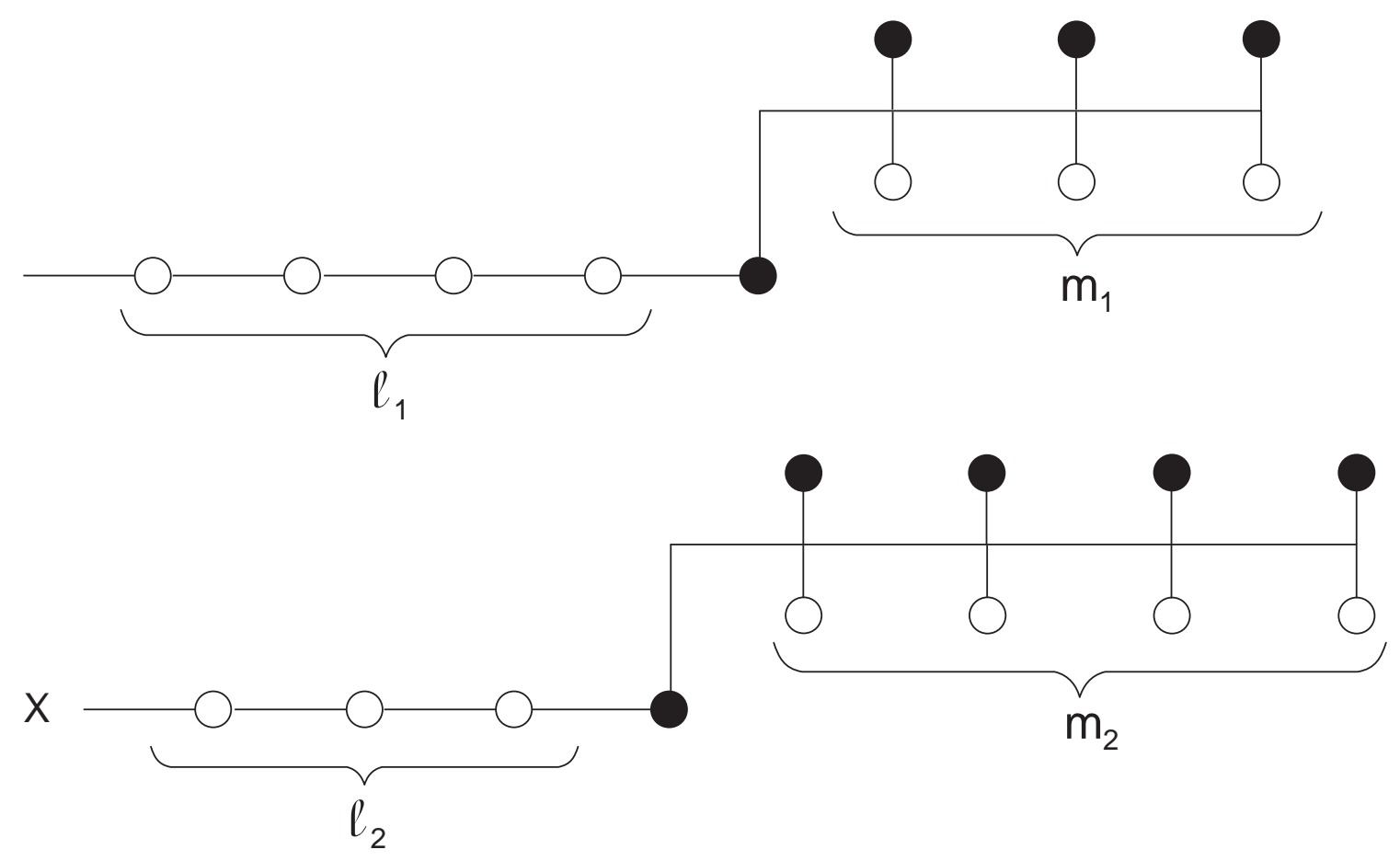
FIGURE 16

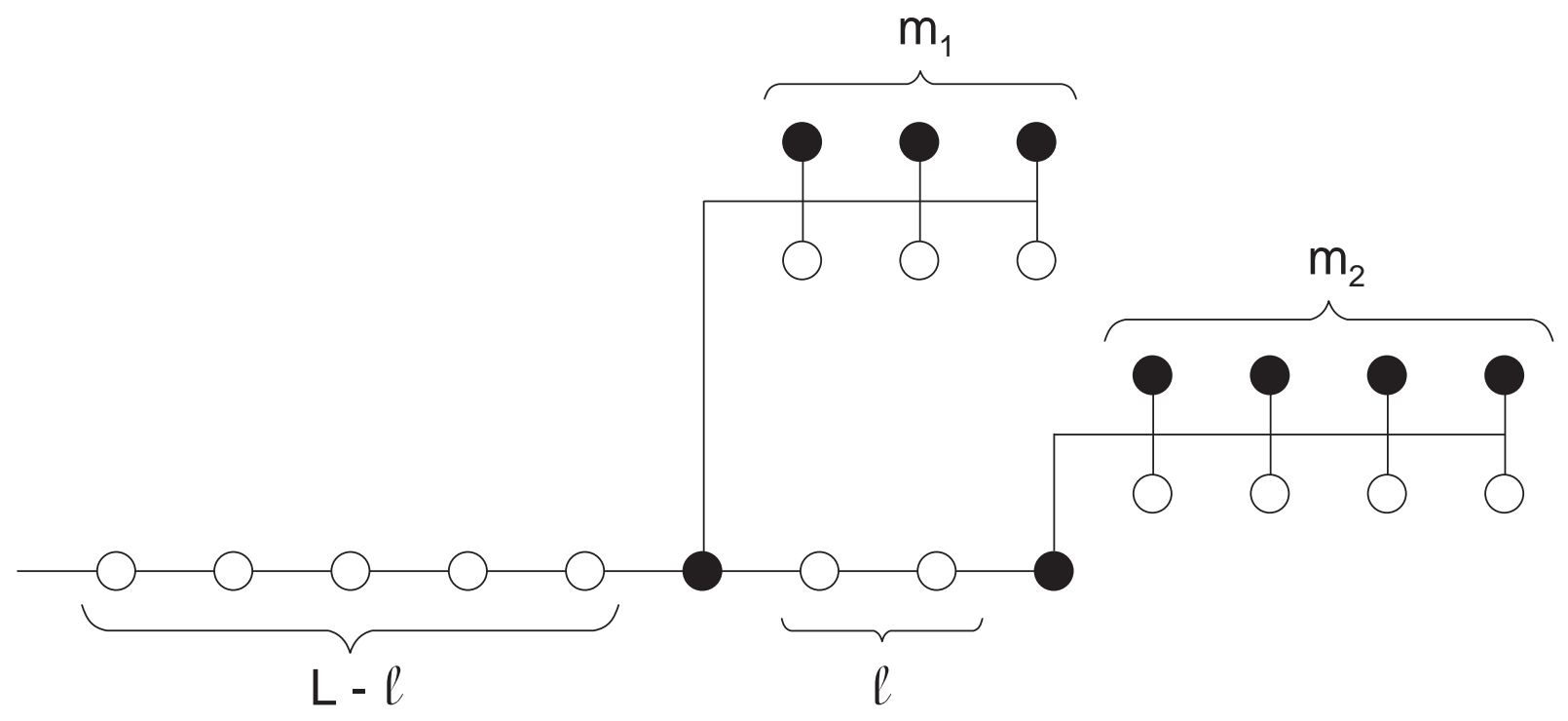

FIGURE 17

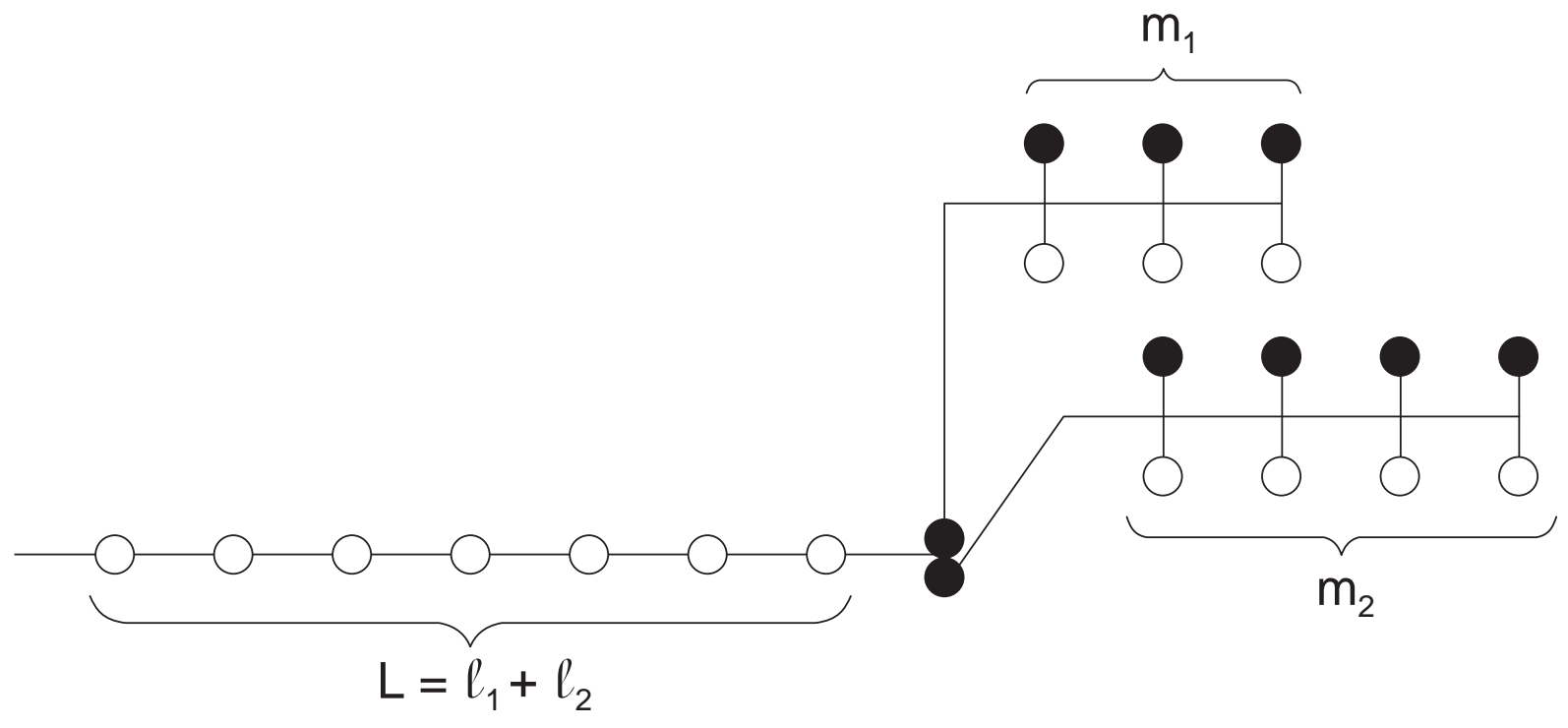


FIGURE 18

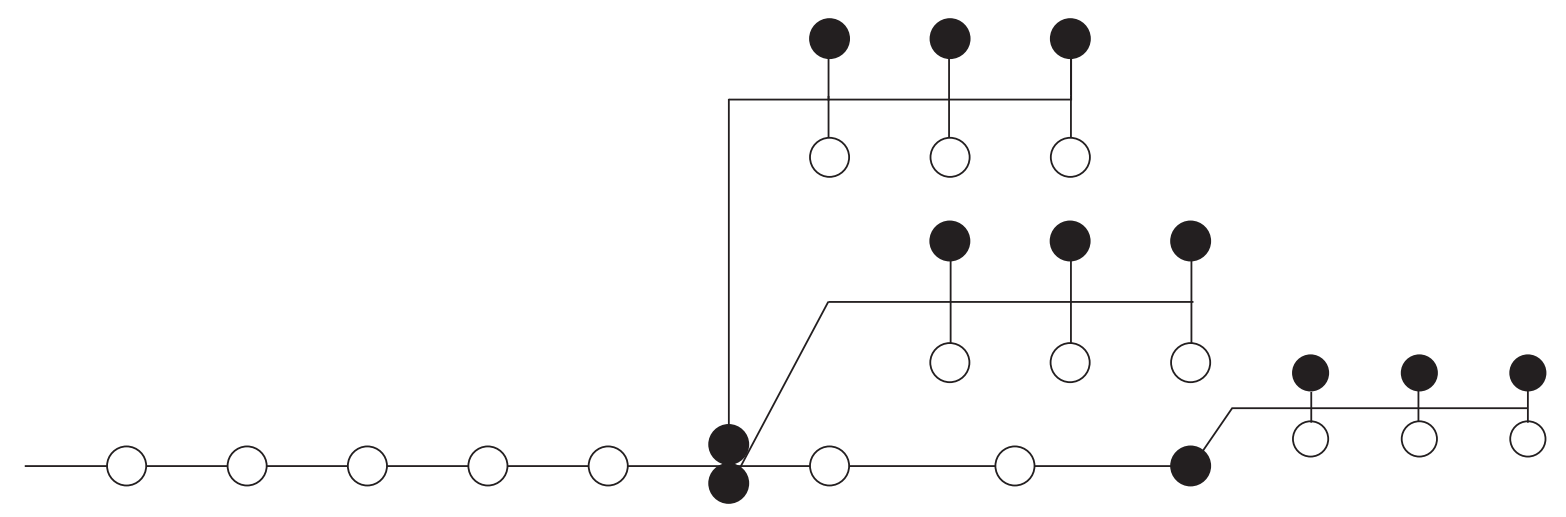

FIGURE 19

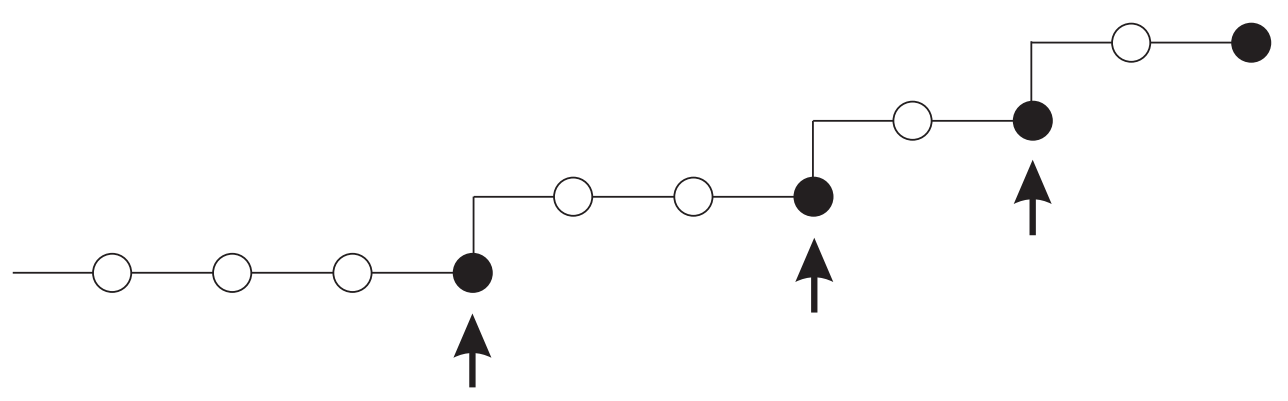


FIGURE 20
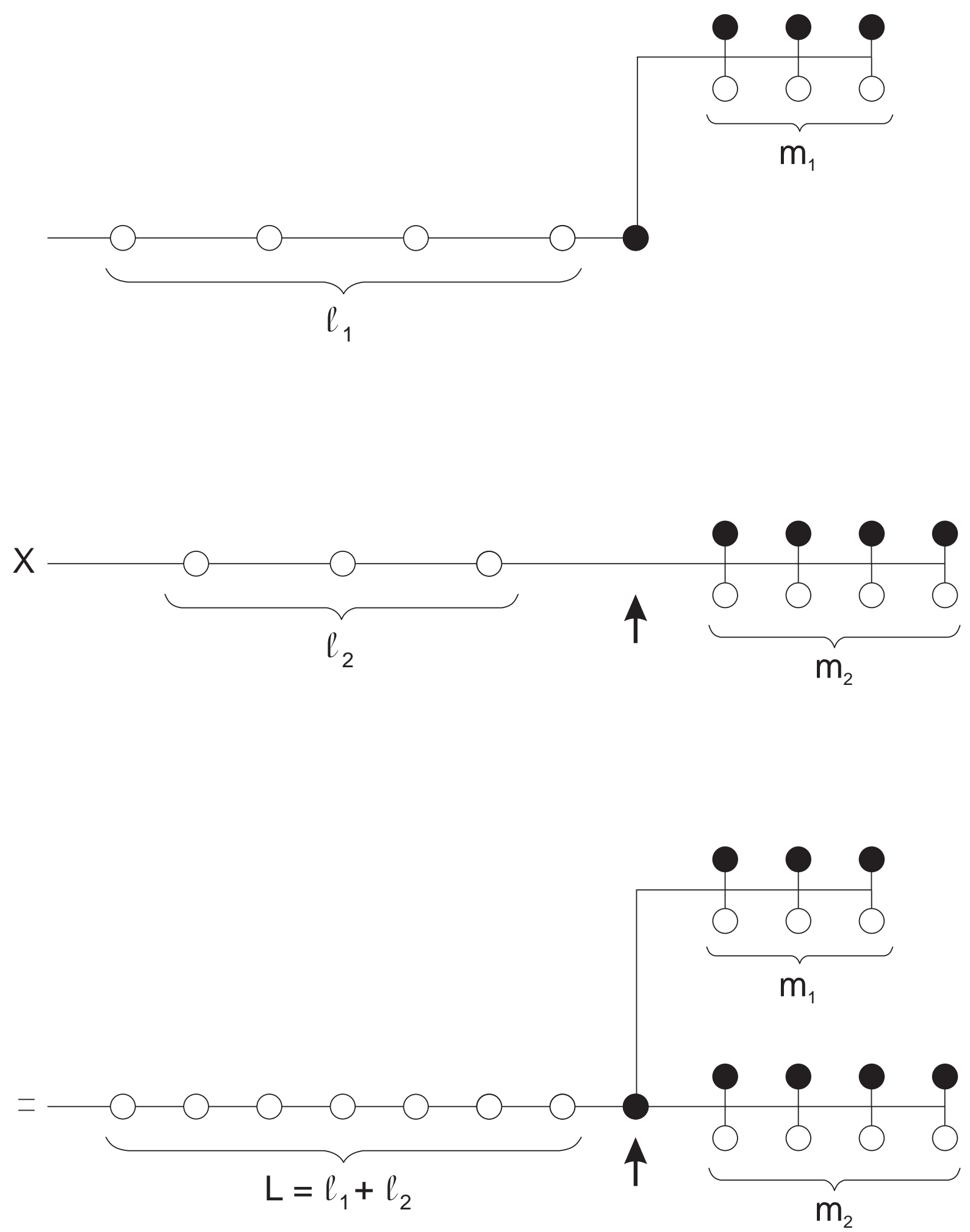
FIGURE 21

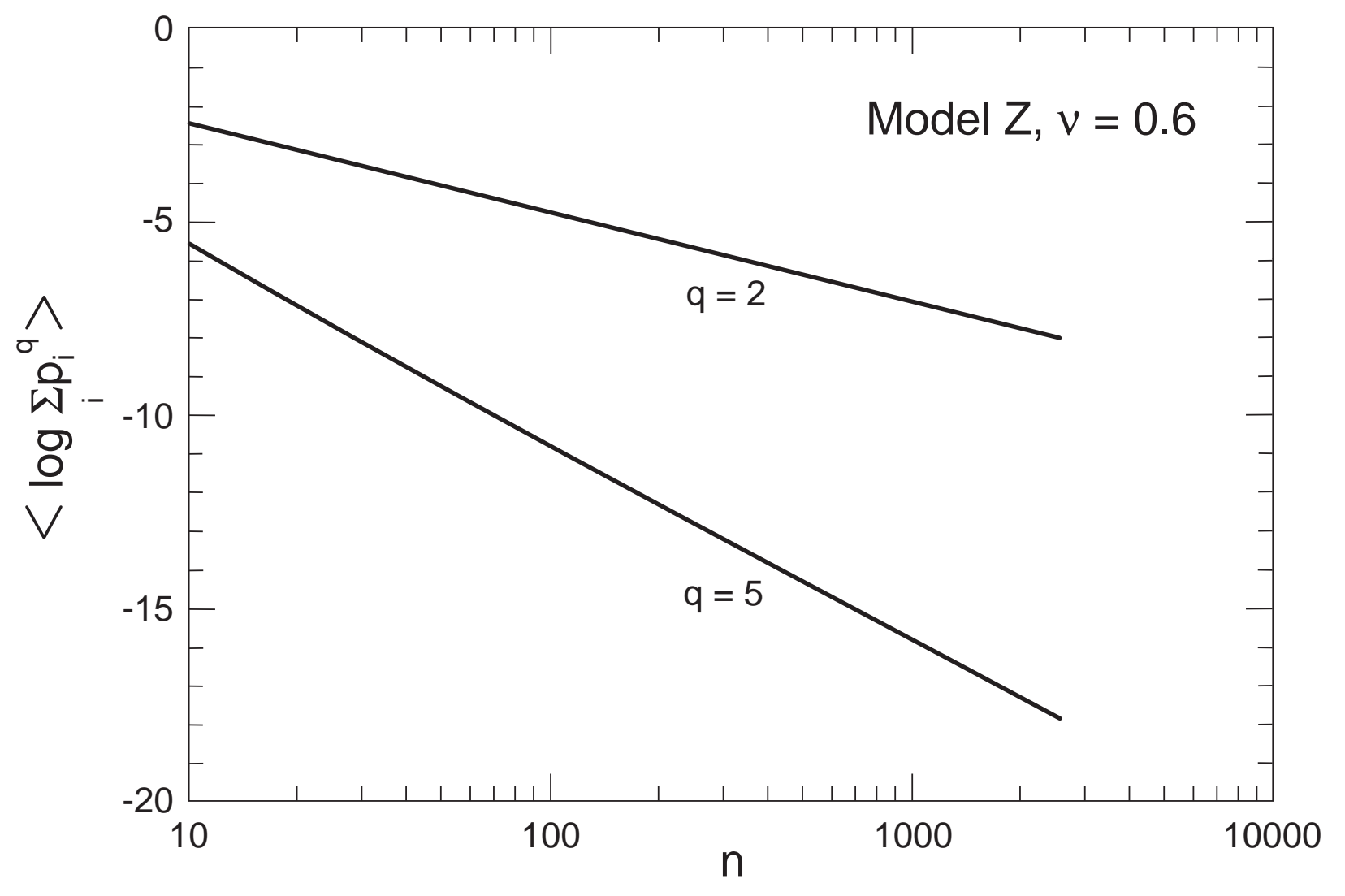


FIGURE 22(a)

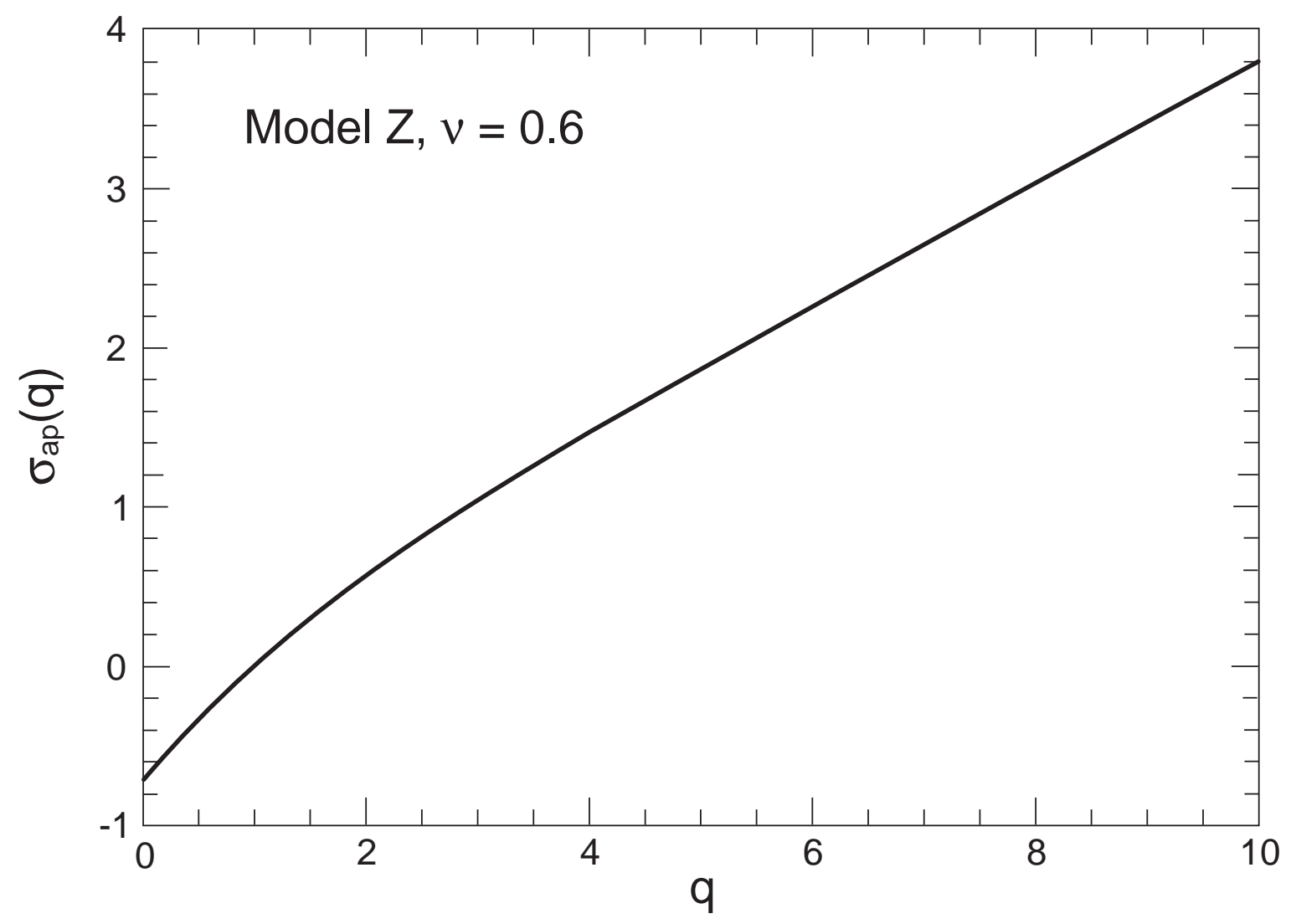


FIGURE 22(b)

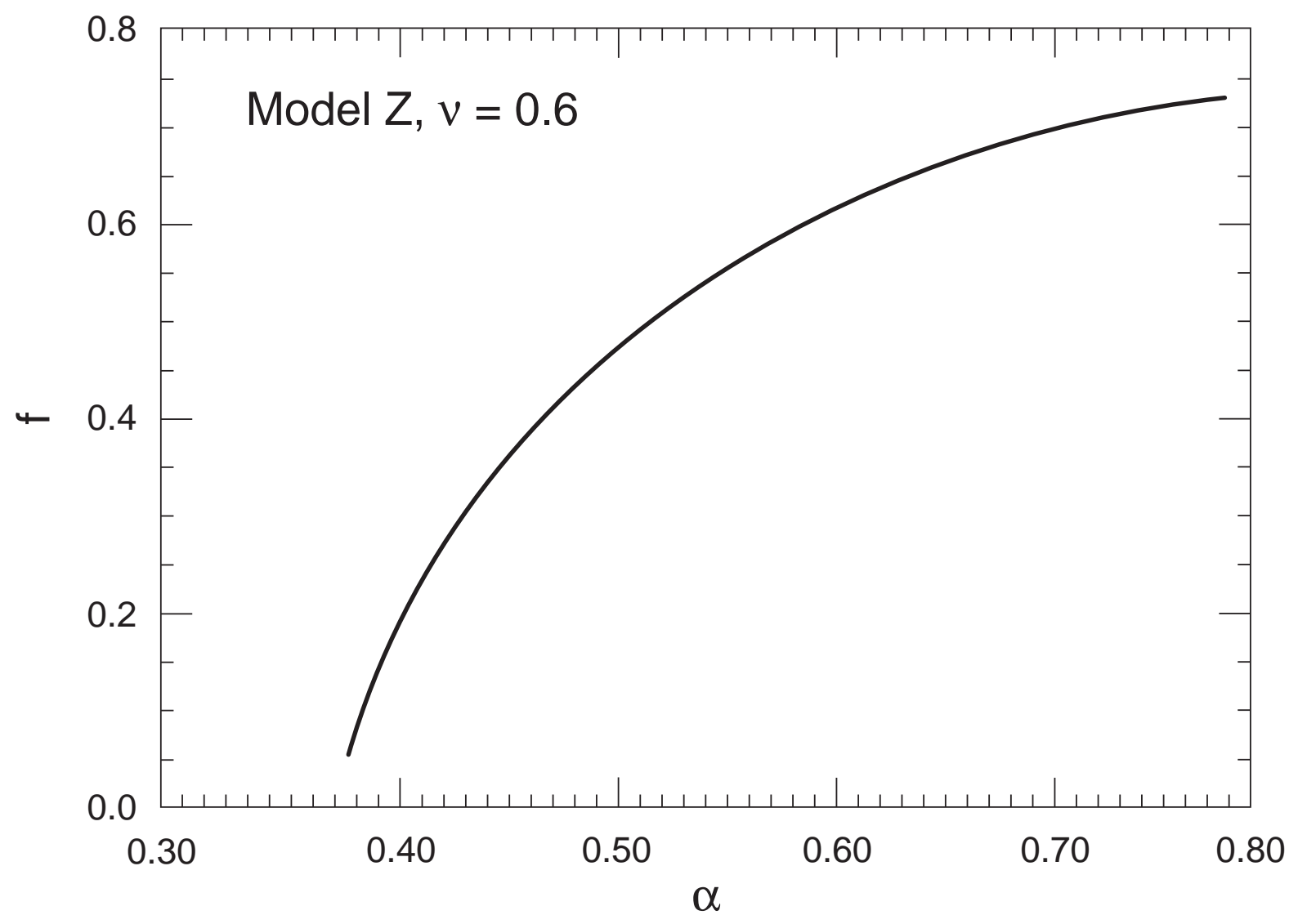


FIGURE 23

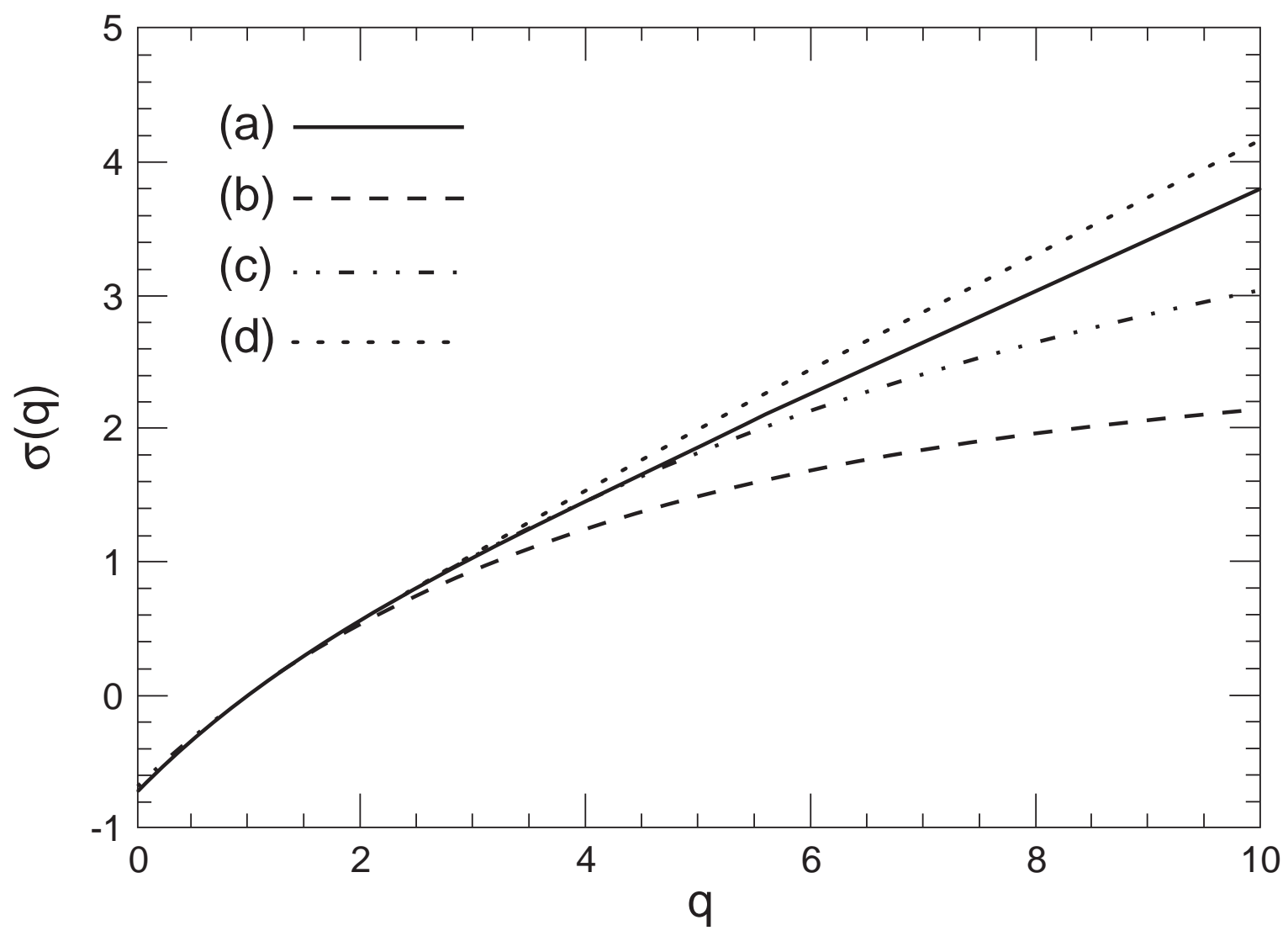


FIGURE 24

$$
\begin{aligned}
& (-\infty+\cdots) \\
& +(-5+-0+0+0) \\
& -\frac{1}{2}(2-0+2-0+2-0 \quad 0+2+0 \\
& +2+2-8+\frac{8}{8}
\end{aligned}
$$


FIGURE 25

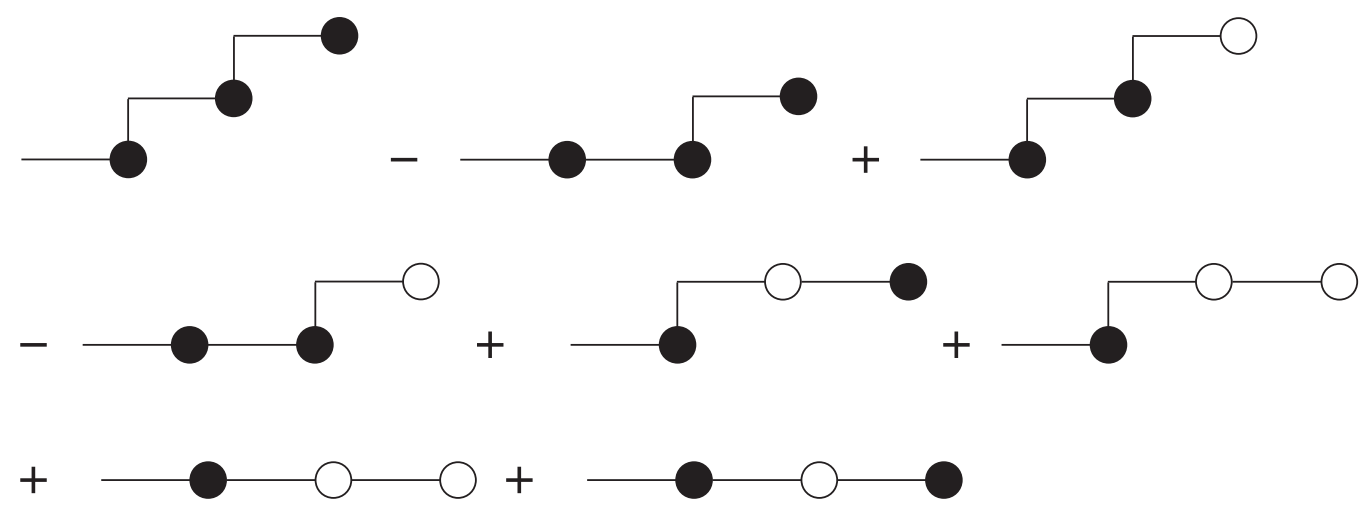

$+2 \bigcirc \quad 0+2$

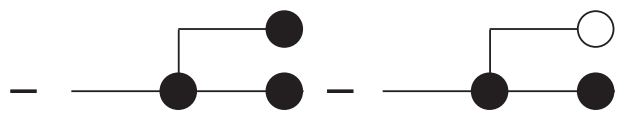

$-\longrightarrow-10$

$+0+0-10-10$

$+2-2+0-10$

$+2-10$

$+2+0-0-10$

$+\frac{1}{3} \longrightarrow+2+\frac{1}{3}+\frac{8}{3}+\frac{1}{3}$ 